\title{
Classification of Finite Dimensional Modular Lie Superalgebras with Indecomposable Cartan Matrix ${ }^{\star}$
}

\author{
Sof iane BOUARROUDJ ${ }^{\dagger}$, Pavel GROZMAN ${ }^{\ddagger}$ and Dimitry LEITES ${ }^{\S}$ \\ ${ }^{\dagger}$ Department of Mathematics, United Arab Emirates University, \\ Al Ain, PO. Box: 17551, United Arab Emirates \\ E-mail: Bouarroudj.sofiane@uaeu.ac.ae \\ ¥ Equa Simulation AB, Stockholm, Sweden \\ E-mail: pavel.grozman@bredband.net \\ $\S$ Department of Mathematics, University of Stockholm, \\ Roslagsv. 101, Kräftriket hus 6, SE-106 91 Stockholm, Sweden \\ E-mail: mleites@math.su.se
}

Received September 17, 2008, in final form May 25, 2009; Published online June 11, 2009 doi:10.3842/SIGMA.2009.060

\begin{abstract}
Finite dimensional modular Lie superalgebras over algebraically closed fields with indecomposable Cartan matrices are classified under some technical, most probably inessential, hypotheses. If the Cartan matrix is invertible, the corresponding Lie superalgebra is simple otherwise the quotient of the derived Lie superalgebra modulo center is simple (if its rank is greater than 1). Eleven new exceptional simple modular Lie superalgebras are discovered. Several features of classic notions, or notions themselves, are clarified or introduced, e.g., Cartan matrix, several versions of restrictedness in characteristic 2, Dynkin diagram, Chevalley generators, and even the notion of Lie superalgebra if the characteristic is equal to 2. Interesting phenomena in characteristic 2: (1) all simple Lie superalgebras with Cartan matrix are obtained from simple Lie algebras with Cartan matrix by declaring several (any) of its Chevalley generators odd; (2) there exist simple Lie superalgebras whose even parts are solvable. The Lie superalgebras of fixed points of automorphisms corresponding to the symmetries of Dynkin diagrams are also listed and their simple subquotients described.
\end{abstract}

Key words: modular Lie superalgebra, restricted Lie superalgebra; Lie superalgebra with Cartan matrix; simple Lie superalgebra

2000 Mathematics Subject Classification: 17B50; 70F25

\section{Contents}

1 Introduction $\quad 2$

2 Basics: Linear algebra in superspaces (from [39]) 5

3 What the Lie superalgebra in characteristic 2 is (from [34]) 9

4 What $\mathfrak{g}(A)$ is $\quad 11$

$5 \quad$ Restricted Lie superalgebras $\quad 15$

6 Ortho-orthogonal and periplectic Lie superalgebras $\quad 18$

${ }^{\star}$ This paper is a contribution to the Special Issue on Kac-Moody Algebras and Applications. The full collection is available at http://www.emis.de/journals/SIGMA/Kac-Moody_algebras.html 
7 Dynkin diagrams $\quad 21$

8 A careful study of an example $\quad 24$

9 Main steps of our classification $\quad 26$

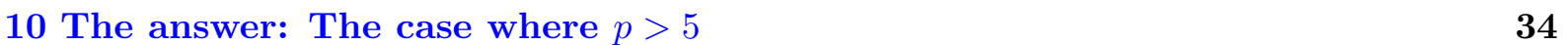

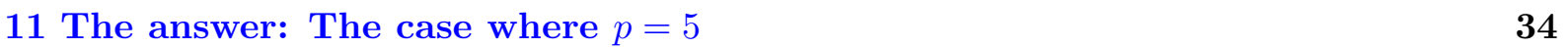

12 The answer: The case where $p=3 \quad 35$

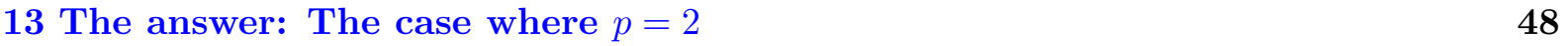

14 Table. Dynkin diagrams for $p=2$

15 Fixed points of symmetries of the Dynkin diagrams $\quad 56$

$\begin{array}{ll}16 \text { A realization of } \mathfrak{g}=\mathfrak{o o}(4 \mid 4)^{(1)} / \mathfrak{c} & \mathbf{5 8}\end{array}$

$\begin{array}{ll}\text { References } & 61\end{array}$

\section{Introduction}

The ground field $\mathbb{K}$ is algebraically closed of characteristic $p>0$. (Algebraic closedness of $\mathbb{K}$ is only needed in the quest for parametric families.) The term "Cartan matrix" will often be abbreviated to CM. Let the size of the square $n \times n$ matrix be equal to $n$.

Except the last section, we will only consider indecomposable Cartan matrices $A$ with entries in $\mathbb{K}$ (so the integer entries are considered as elements of $\mathbb{Z} / p \mathbb{Z} \subset \mathbb{K}$ ), indecomposability being the two conditions:

1. $A_{i j}=0 \Longleftrightarrow A_{j i}=0$.

2. By a reshuffle of its rows and columns $A$ can not be reduced to a block-diagonal form.

\subsection{Main results}

1. We clarify several key notions - of Lie superalgebra in characteristic 2, of Lie superalgebra with Cartan matrix, of weights and roots.

2. We introduce several versions of restrictedness for Lie (super)algebras in characteristic 2 . These clarifications are obtained by/with A. Lebedev.

3. We give an algorithm that, under certain (conjecturally immaterial) hypotheses, see Section 9.2.5), produces the complete list of all finite dimensional Lie superalgebras possessing indecomposable Cartan matrices $A$, i.e., of the form $\mathfrak{g}(A)$. Our proof follows the same lines Weisfeiler and Kac outlined for the Lie algebra case in [54]. The result of application of the algorithm - the classification - is summarized in the following theorem:

Theorem (Sections 10-13).

1. There are listed all finite dimensional Lie superalgebras (which are not Lie algebras) of the form $\mathfrak{g}(A)$ with indecomposable $A$ over $\mathbb{K}$.

2. There are listed all inequivalent systems of simple roots (inequivalent Cartan matrices) of each of the above listed Lie superalgebras $\mathfrak{g}(A)$. 
3. For the above listed Lie superalgebras $\mathfrak{g}=\mathfrak{g}(A)$, their even parts $\mathfrak{g}_{0}$ and the $\mathfrak{g}_{0}$-modules $\mathfrak{g}_{\overline{1}}$ are explicitly described in terms of simple and solvable Lie algebras and modules over them.

4. For the above listed Lie superalgebras $\mathfrak{g}=\mathfrak{g}(A)$, the existence of restrictedness is explicitly established; for $p=2$, various cases of restrictedness are considered and explicit formulas given in each case.

The results for $p>5, p=5,3$ and 2 are summarized in Sections 10, 11, 12 and 13, respectively.

The following simple Lie superalgebras (where $\mathfrak{g}$ is the Lie superalgebra with Cartan matrix and $\mathfrak{g}^{(1)} / \mathfrak{c}$ is the quotient of its first derived algebra modulo the center) are new:

1) $\mathfrak{e}(6,1), \mathfrak{e}(6,6), \mathfrak{e}(7,1)^{(1)} / \mathfrak{c}, \mathfrak{e}(7,6)^{(1)} / \mathfrak{c}, \mathfrak{e}(7,7)^{(1)} / \mathfrak{c}, \mathfrak{e}(8,1), \mathfrak{e}(8,8) ; \mathfrak{b} \mathfrak{g l}(4 ; \alpha)$ and $\mathfrak{b} \mathfrak{g l}(3 ; \alpha)^{(1)} / \mathfrak{c}$ for $p=2$;

2) $\mathfrak{e l}(5 ; 3)$ for $p=3$;

3) $\mathfrak{b r j}(2 ; 5)$ for $p=5$.

Observe that although several of the exceptional examples were known for $p>2$, together with one indecomposable Cartan matrix per each Lie superalgebra [17, 18, 13, 14], the complete description of all inequivalent Cartan matrices for all the exceptional Lie superalgebras of the form $\mathfrak{g}(A)$ and for ALL cases for $p=2$ is new.

A posteriori we see that for each finite dimensional Lie superalgebra $\mathfrak{g}(A)$ with indecomposable Cartan matrix, the module $\mathfrak{g}_{\overline{1}}$ is a completely reducible $\mathfrak{g}_{\overline{0}}$-module ${ }^{1}$.

\section{Fixed points of the symmetries of Dynkin diagrams}

We also listed the Lie superalgebras of fixed points of automorphisms corresponding to the symmetries of Dynkin diagrams and described their simple subquotients. In characteristic 0, this is the way all Lie algebras whose Dynkin diagrams has multiple bonds (roots of different lengths) are obtained. Since, for $p=2$, there are no multiple bonds or roots of different length (at least, this notion is not invariant), it is clear that this is the way to obtain something new, although, perhaps, not simple. Lemma 2.2 in [23] implicitly describes the ideal in the Lie algebra of fixed points of an automorphism of a Lie algebra, but one still has to describe the Lie algebra of fixed points explicitly. This explicit answer is given in the last section. No new simple Lie (super)algebras are obtained.

\subsubsection{On simple subquotients of Lie (super)algebras of the form $\mathfrak{g}(A)$ and a terminological problem}

Observe that if a given indecomposable Cartan matrix $A$ is invertible, the Lie (super)algebra $\mathfrak{g}(A)$ is simple, otherwise $\mathfrak{g}(A)^{(i)} / \mathfrak{c}$ - the quotient of its first (if $i=1$ ) or second (if $i=2$ ) derived algebra modulo the center - is simple (if $\operatorname{size} A>1$ ). Hereafter in this situation $i=1$ or 2 ; meaning that the chain of derived algebras stabilizes $\left(\mathfrak{g}(A)^{(j)} \simeq \mathfrak{g}(A)^{(i)}\right.$ for any $\left.j \geq i\right)$. We will see a posteriori that $\operatorname{dim} \mathfrak{c}=i=\operatorname{corank} A$, the maximal possible value of $i$ above.

This simple Lie algebra $\mathfrak{g}(A)^{(i)} / \mathfrak{c}$ does NOT possess any Cartan matrix. Except for Lie algebras over fields of characteristic $p=0$, this subtlety is never mentioned causing confusion: The conventional sloppy practice is to refer to the simple Lie (super)algebra $\mathfrak{g}(A)^{(i)} / \mathfrak{c}$ as "possessing a Cartan matrix" (although it does not possess any) and at the same time to say " $\mathfrak{g}(A)$ is simple" whereas it is not. However, it is indeed extremely inconvenient to be completely correct,

\footnotetext{
${ }^{1}$ For the simple subquotient $\mathfrak{g}=\mathfrak{g}(A)^{(i)} / \mathfrak{c}$ of $\mathfrak{g}(A)$, where $i=$ corank $A$ is equal to 1 or 2 , complete reducibility of the $\mathfrak{g}_{\overline{0}}$-module $\mathfrak{g}_{\overline{1}}$ is sometimes violated.
} 
especially in the cases where $\mathfrak{g}(A)$, as well as its simple subquotient $\mathfrak{g}(A)^{(i)} / \mathfrak{c}$, and a central extension of the latter, the algebra $\mathfrak{g}(A)^{(i)}$, or all three at the same time might be needed. So we suggest to refer to either of these three algebras as "simple" ones, and extend the same convention to Lie superalgebras.

Thus, for $i=1$, there are three distinct Lie (super)algebras: $\mathfrak{g}(A)$ with Cartan matrix, $\mathfrak{g}(A)^{(1)}$ its derived, and $\mathfrak{g}(A)^{(1)} / \mathfrak{c}$ which is simple. It sometimes happens that the three versions are needed at the same time and to appropriately designate them is a non-trivial task dealt with in Section 4. When the second derived of $\mathfrak{g}(A)$ is not isomorphic to the first one, i.e., when $i=2$, there are even more intermediate derived objects, but fortunately only the three of them $-\mathfrak{g}(A)$ with Cartan matrix, $\mathfrak{g}(A)^{(2)}$, and $\mathfrak{g}(A)^{(2)} / \mathfrak{c}$, the quotient modulo the whole center - appear (so far) in applications.

\subsubsection{The Elduque Supermagic Square}

For $p>2$, Elduque interpreted most of the exceptional (when their exceptional nature was only conjectured; now this is proved) simple Lie superalgebras (of the form $\mathfrak{g}(A)$ ) in characteristic 3 [14] in terms of super analogs of division algebras and collected them into a Supermagic Square (an analog of Freudenthal's Magic Square). The rest of the exceptional examples for $p=3$ and $p=5$, not entering the Elduque Supermagic Square (the ones described here for the first time) are, nevertheless, somehow affiliated to the Elduque Supermagic Square [19].

\subsection{Characteristic 2}

Very interesting, we think, is the situation in characteristic 2. A posteriori we see that the list of Lie superalgebras in characteristic 2 of the form $\mathfrak{g}(A)$ or $\mathfrak{g}(A)^{(i)} / \mathfrak{c}$, where $i=$ corank $A$ can be equal to either 1 or 2 , with an indecomposable matrix $A$ is as follows:

1. Take any finite dimensional Lie algebra of the form $\mathfrak{g}(A)$ with indecomposable Cartan matrix [54] and declare some of its Chevalley generators (simultaneously a positive one and the respective negative one) odd (the corresponding diagonal elements of $A$ should be changed accordingly: $\overline{0}$ to 0 and $\overline{1}$ to 1 , see Section 4.5 ). Let $I$ be the vector of parities of the generators; the parity of each positive generator should equal to that of the corresponding negative one.

2. Do this for each of the inequivalent Cartan matrices of $\mathfrak{g}(A)$ and any distribution $I$ of parities.

3. Construct Lie superalgebra $\mathfrak{g}(A, I)$ from these Chevalley generators by factorizing a certain $\widetilde{\mathfrak{g}}(A, I)$ modulo an ideal (explicitly described in [37, 3, 4]; for a summary, see [2]).

4. For the Lie superalgebra $\mathfrak{g}(A, I)$, list all its inequivalent Cartan matrices.

5. If $A$ is not invertible, pass to $\mathfrak{g}^{(i)}(A, I) / \mathfrak{c}$.

Such superization turns

1) a given orthogonal Lie algebra into either an ortho-orthogonal or a periplectic Lie superalgebra;

2) the three exceptional Lie algebras of $\mathfrak{e}$ type turn into seven non-isomorphic Lie superalgebras of $\mathfrak{e}$ type;

3) the $\mathfrak{w k}$ type Lie algebras discovered in [54] turn into $\mathfrak{b} \mathfrak{g l}$ type Lie superalgebras. 
1.3. Remarks. 1) Observe that the Lie (super)algebra uniformly defined for any characteristic as

- preserving a tensor, e.g., (super)trace-less; (ortho-)orthogonal and periplectic ones,

- given by an integer Cartan matrix whose entries are considered modulo $p$

may have different (super)dimensions as $p$ varies (not only from 2 to "not 2"): The "same" Cartan matrices might define algebras of similar type but with different properties and names as the characteristic changes: for example $\mathfrak{s l}(n p)$ has $\mathrm{CM}$ in all characteristics, except $p$, in which case it is $\mathfrak{g l}(n p)$ that has a CM.

2) Although the number of inequivalent Cartan matrices grows with the size of $A$, it is easy to list all possibilities for serial Lie (super)algebras. Certain exceptional Lie superalgebras have dozens of inequivalent Cartan matrices; nevertheless, there are at least the following reasons to list all of them:

1. To classify all $\mathbb{Z}$-gradings of a given $\mathfrak{g}(A)$ (in particular, inequivalent Cartan matrices) is a very natural problem. Besides, sometimes the knowledge of the best, for the occasion, $\mathbb{Z}$-grading is important. Examples of different cases: [44] (all simple roots non-isotropic), [38] (all simple roots odd); for computations "by hand" the cases where only one simple root is odd are useful.

In particular, the defining relations between the natural (Chevalley) generators of $\mathfrak{g}(A)$ are of completely different form for inequivalent $\mathbb{Z}$-gradings, and this is used in [44].

2. Distinct $\mathbb{Z}$-gradings yield distinct Cartan-Tanaka-Shchepochkina (CTS) prolongs (vectorial Lie (super)algebras), cf. [49,35]. So to classify them is vital, for example, in the quest for simple vectorial Lie (super)algebras.

3. Certain properties of Cartan matrices may vary under the passage from one inequivalent CM to the other (the Lie superalgebras that correspond to such matrices may have different rate of growth as $\mathbb{Z}$-graded algebras); this is a novel, previously unnoticed, feature of Lie superalgebras that had lead to false claims (rectified in [11]).

\subsection{Related results}

1) For explicit presentations in terms of (the analogs of) Chevalley generators of the Lie algebras and superalgebras listed here, see [2]. In addition to Serre-type relations there always are more complicated relations.

2) For deformations of the finite dimensional Lie (super)algebras of the form $\mathfrak{g}(A)$ and $\mathfrak{g}(A)^{(i)} / \mathfrak{c}$, see [6]. Observe that whereas "for $p>3$, the Lie algebras with Cartan matrices of the same types that exist over $\mathbb{C}$ are either rigid or have deforms which also possess Cartan matrices", this is not so if $p=3$ or 2 .

3) For generalized Cartan-Tanaka-Shchepochkina (CTS) prolongs of the simple Lie (super)algebras of the form $\mathfrak{g}(A)$, and the simple subquotients of such prolongs, see [5, 7].

4) With restricted Lie algebras one can associate algebraic groups; analogously, with restricted Lie superalgebras one can associate algebraic supergroups. For this and other results of Lebedev's Ph.D. thesis pertaining to the classification of simple modular Lie superalgebras, see [37].

\section{Basics: Linear algebra in superspaces (from [39])}

\subsection{General notation}

For further details on basics on Linear Algebra in Superspaces, see [39]. 
For the definition of the term "Lie superalgebra", especially, in characteristic 2, see Section 3.

For the definition of the Lie (super)algebras of the form $\mathfrak{g}(A)$, i.e., with Cartan matrix $A$ (briefly referred to CM Lie (super)algebras), and simple relatives of the Lie superalgebras of the form $\mathfrak{g}(A)$ with indecomposable Cartan matrix $A$, see Section 4. For simplicity of typing, the $i$ th incarnation of the Lie (super)algebra $\mathfrak{g}(A)$ with the $i$ th $\mathrm{CM}$ (according to the lists given below) will be denoted by $i \mathfrak{g}(A)$.

For the split form of any simple Lie algebra $\mathfrak{g}$, we denote the $\mathfrak{g}$-module with the $i$ th fundamental weight $\pi_{i}$ by $R\left(\pi_{i}\right)$ (as in [41, 8]; these modules are denoted by $\Gamma_{i}$ in [24]). In particular, for $\mathfrak{o}(2 k+1)$, the spinor representation $\operatorname{spin}_{2 k+1}$ is defined to be the $k$ th fundamental representation, whereas for $\mathfrak{o}_{\Pi}(2 k)$, the spinor representations are the $k$ th and the $(k-1)$ st fundamental representations. The realizations of these representations and the corresponding modules by means of quantization (as in [40]) can also be defined, since, as is easy to see, the same quantization procedure is well-defined for the restricted version of the Poisson superalgebra. (Fortunately, we do not need irreducible representations of $\mathfrak{o}_{\mathrm{I}}(2 k)$; their description is unknown, except the trivial, identity and adjoint ones.)

In what follows, ad denotes both the adjoint representation and the module in which it acts. Similarly, id denotes both the identity (a.k.a. standard) representation of the linear Lie (super)algebra $\mathfrak{g} \subset \mathfrak{g l}(V)$ in the (super)space $V$ and $V$ itself. (We disfavor the adjective "natural" applied to id only, since it is no less appropriate to any of the tensor (symmetric, exterior) powers of id.) In particular, having fixed a basis in the $n$-dimensional space and having realized $\mathfrak{s l}(V)$ as $\mathfrak{s l}(n)$, we write id instead of $V$, so $V$ does not explicitly appear. The exterior powers and symmetric powers of the vector space $V$ are defined as quotients of its tensor powers

$$
\begin{aligned}
& T^{0}(V):=\bigwedge^{0}(V):=S^{0}(V):=\mathbb{K}, \\
& T^{1}(V):=\bigwedge_{1}(V):=S^{1}(V):=V, \\
& T^{i}(V):=\underbrace{V \otimes \cdots \otimes V}_{i \text { factors }} \quad \text { for } i>0 .
\end{aligned}
$$

We set:

$$
\wedge^{\bullet}(V):=T^{\bullet}(V) /(x \otimes x \mid x \in V), \quad S^{\bullet}(V):=T^{\bullet}(V) /(x \otimes y+y \otimes x \mid x, y \in V),
$$

where $T^{\bullet}(V):=\oplus T^{i}(V)$; let $\bigwedge^{i}(V)$ and $S^{i}(V)$ be homogeneous components of degree $i$.

Describing the $\mathfrak{g}_{\overline{0}}$-module structure of $\mathfrak{g}_{\overline{1}}$ for a Lie superalgebra $\mathfrak{g}$, we write $\mathfrak{g}_{\overline{1}} \simeq R(\cdot)$, though it is, actually, $\Pi(R(\cdot))$.

The symbol $A \in B$ denotes a semi-direct sum of modules of which $A$ is a submodule; when dealing with algebras, $A$ is an ideal in $A \in B$.

\subsection{Superspaces}

A superspace is a $\mathbb{Z} / 2$-graded space; for any superspace $V=V_{\overline{0}} \oplus V_{\overline{1}}$, where $\overline{0}$ and $\overline{1}$ are residues modulo 2 ; we denote by $\Pi(V)$ another copy of the same superspace: with the shifted parity, i.e., $(\Pi(V))_{\bar{i}}=V_{\bar{i}+\overline{1}}$. The parity function is denoted by $p$. The superdimension of $V$ is $\operatorname{sdim} V=a+b \varepsilon$, where $\varepsilon^{2}=1$, and $a=\operatorname{dim} V_{\overline{0}}, b=\operatorname{dim} V_{\overline{1}}$. (Usually, $\operatorname{sdim} V$ is shorthanded as a pair $(a, b)$ or $a \mid b$; this notation obscures the fact that $\operatorname{sdim} V \otimes W=\operatorname{sdim} V \cdot \operatorname{sdim} W$.)

A superspace structure in $V$ induces the superspace structure in the space $\operatorname{End}(V)$. A basis of a superspace is always a basis consisting of homogeneous vectors; let $\operatorname{Par}=\left(p_{1}, \ldots, p_{\operatorname{dim} V}\right)$ be an ordered collection of their parities. We call Par the format of (the basis of) $V$. A square supermatrix of format (size) Par is a $\operatorname{sdim} V \times \operatorname{sdim} V$ matrix whose $i$ th row and $i$ th column are of the same parity $p_{i} \in$ Par. The matrix unit $E_{i, j}$ is of parity $p_{i}+p_{j}$. 
Whenever possible, we consider one of the simplest formats Par, e.g., the format $\operatorname{Par}_{s t}$ of the form $(\overline{0}, \ldots, \overline{0} ; \overline{1}, \ldots, \overline{1})$ is called standard. Systems of simple roots of Lie superalgebras corresponding to distinct nonstandard formats of supermatrix realizations of these superalgebras are related by so-called odd reflections.

\subsection{The Sign Rule}

The formulas of Linear Algebra are superized by means of the Sign Rule:

if something of parity $p$ moves past something of parity $q$, the sign $(-1)^{p q}$

accrues; the expressions defined on homogeneous elements are extended

to arbitrary ones via linearity.

Examples of application of the Sign Rule: By setting

$$
[X, Y]=X Y-(-1)^{p(X) p(Y)} Y X
$$

we get the notion of the supercommutator and the ensuing notions of supercommutative and superanti-commutative superalgebras; Lie superalgebra is the one which, in addition to superanticommutativity, satisfies Jacobi identity amended with the Sign Rule; a superderivation of a given superalgebra $A$ is a linear map $D: A \longrightarrow A$ satisfying the super Leibniz rule

$$
D(a b)=D(a) b+(-1)^{p(D) p(a)} a D(b) .
$$

Let $V$ be a superspace, $\operatorname{sdim} V=m \mid n$. The general linear Lie superalgebra of operators acting in $V$ is denoted by $\mathfrak{g l}(V)$ or $\mathfrak{g l}(m \mid n)$ if an homogeneous basis of $V$ is fixed. The Lie subsuperalgebra of supertraceless operators (supermatrices) is denoted $\mathfrak{s l}(V) \simeq \mathfrak{s l}(m \mid n)$. If a Lie superalgebra $\mathfrak{g} \subset \mathfrak{g l}(V)$ contains the ideal of scalar matrices $\mathfrak{s}=\mathbb{K} 1_{m \mid n}$, then $\mathfrak{p g}=\mathfrak{g} / \mathfrak{s}$ denotes the projective version of $\mathfrak{g}$.

Observe that sometimes the Sign Rule requires some dexterity in application. For example, we have to distinguish between super-skew and super-anti although both versions coincide in the non-super case:

$$
\begin{array}{llrl}
b a & =(-1)^{p(b) p(a)} a b & & \text { (supercommutativity), } \\
b a & =-(-1)^{p(b) p(a)} a b & & \text { (superanti-commutativity), } \\
b a & =(-1)^{(p(b)+1)(p(a)+1)} a b & & \text { (superskew-commutativity), } \\
b a & =-(-1)^{(p(b)+1)(p(a)+1)} a b & & \text { (superantiskew-commutativity). }
\end{array}
$$

In other words, "anti" means the total change of the sign, whereas any "skew" notion can be straightened by the parity change. In what follows, the supersymmetric bilinear forms and supercommutative superalgebras are named according to the above definitions.

The supertransposition of supermatrices is defined so as to assign the supertransposed supermatrix to the dual operator in the dual bases. For details, see [36]; an explicit expression in the standard format is as follows (we give a general formula for matrices with entries in a supercommutative superalgebra; in this paper we only need the lower formula):

$$
X=\left(\begin{array}{ll}
a & b \\
c & d
\end{array}\right) \mapsto X^{s t}:= \begin{cases}\left(\begin{array}{cc}
a^{t} & c^{t} \\
-b^{t} & d^{t}
\end{array}\right) & \text { for } X \text { even } \\
\left(\begin{array}{cc}
a^{t} & -c^{t} \\
b^{t} & d^{t}
\end{array}\right) & \text { for } X \text { odd }\end{cases}
$$




\subsection{What the Lie superalgebra is}

Dealing with superalgebras it sometimes becomes useful to know their definition. Lie superalgebras were distinguished in topology in 1930's, and the Grassmann superalgebras half a century earlier. So it might look strange when somebody offers a "better" definition of a notion which was established about 70 year ago. Nevertheless, the answer to the question "what is a (Lie) superalgebra?" is still not a common knowledge.

So far we defined Lie superalgebras naively: via the Sign Rule. However, the naive definition suggested above ("apply the Sign Rule to the definition of the Lie algebra") is manifestly inadequate for considering the supervarieties ${ }^{2}$ of deformations and for applications of representation theory to mathematical physics, for example, in the study of the coadjoint representation of the Lie supergroup which can act on a supermanifold or supervariety but never on a vector superspace - an object from another category. We were just lucky in the case of finite dimensional Lie algebras over $\mathbb{C}$ that the vector spaces can be viewed as manifolds or varieties. In the case of spaces over $\mathbb{K}$ and in the super setting, to be able to deform Lie (super)algebras or to apply group-theoretical methods, we must be able to recover a supermanifold from a vector superspace, and vice versa.

A proper definition of Lie superalgebras is as follows. The Lie superalgebra in the category of supervarieties corresponding to the "naive" Lie superalgebra $L=L_{\overline{0}} \oplus L_{\overline{1}}$ is a linear supermanifold $\mathcal{L}=\left(L_{\overline{0}}, \mathcal{O}\right)$, where the sheaf of functions $\mathcal{O}$ consists of functions on $L_{\overline{0}}$ with values in the Grassmann superalgebra on $L_{\overline{1}}^{*}$; this supermanifold should be such that for "any" (say, finitely generated, or from some other appropriate category) supercommutative superalgebra $C$, the space $\mathcal{L}(C)=\operatorname{Hom}(\operatorname{Spec} C, \mathcal{L})$, called the space of $C$-points of $\mathcal{L}$, is a Lie algebra and the correspondence $C \longrightarrow \mathcal{L}(C)$ is a functor in $C$. (A. Weil introduced this approach in algebraic geometry in 1953; in super setting it is called the language of points or families.) This definition might look terribly complicated, but fortunately one can show that the correspondence $\mathcal{L} \longleftrightarrow L$ is one-to-one and the Lie algebra $\mathcal{L}(C)$, also denoted $L(C)$, admits a very simple description: $L(C)=(L \otimes C)_{\overline{0}}$.

A Lie superalgebra homomorphism $\rho: L_{1} \longrightarrow L_{2}$ in these terms is a functor morphism, i.e., a collection of Lie algebra homomorphisms $\rho_{C}: L_{1}(C) \longrightarrow L_{2}(C)$ such that any homomorphism of supercommutative superalgebras $\varphi: C \longrightarrow C_{1}$ induces a Lie algebra homomorphism $\varphi: L(C) \longrightarrow L\left(C_{1}\right)$ and products of such homomorphisms are naturally compatible. In particular, a representation of a Lie superalgebra $L$ in a superspace $V$ is a homomorphism $\rho: L \longrightarrow \mathfrak{g l}(V)$, i.e., a collection of Lie algebra homomorphisms $\rho_{C}: L(C) \longrightarrow(\mathfrak{g l}(V) \otimes C)_{\overline{0}}$.

2.4.1. Example. Consider a representation $\rho: \mathfrak{g} \longrightarrow \mathfrak{g l}(V)$. The space of infinitesimal deformations of $\rho$ is isomorphic to $H^{1}\left(\mathfrak{g} ; V \otimes V^{*}\right)$. For example, if $\mathfrak{g}$ is the $0 \mid n$-dimensional (i.e., purely odd) Lie superalgebra (with the only bracket possible: identically equal to zero), its only irreducible modules are the trivial one, $\mathbf{1}$, and $\Pi(\mathbf{1})$. Clearly,

$$
\mathbf{1} \otimes 1^{*} \simeq \Pi(\mathbf{1}) \otimes \Pi(\mathbf{1})^{*} \simeq \mathbf{1}
$$

and, because the Lie superalgebra $\mathfrak{g}$ is commutative, the differential in the cochain complex is zero. Therefore $H^{1}(\mathfrak{g} ; \mathbf{1})=\bigwedge^{1}\left(\mathfrak{g}^{*}\right) \simeq \mathfrak{g}^{*}$, so there are $\operatorname{dim} \mathfrak{g}$ odd parameters of deformations of the trivial representation. If we consider $\mathfrak{g}$ "naively", all of these odd parameters will be lost.

Examples that lucidly illustrate why one should always remember that a Lie superalgebra is not a mere linear superspace but a linear supermanifold are, e.g., the deforms with odd parameters. In the category of supervarieties, these deforms, listed in [6], are simple Lie superalgebras.

\footnotetext{
${ }^{2} \mathrm{~A}$ supervariety is a ringed space such that the collection of functions on it - the sections of its sheaf constitute a supercommutative superring. Morphisms of supervarieties are only the ring space morphisms that preserve parity of the superrings of sections of the structure sheaves.
} 


\subsection{Examples of simple Lie superalgebras over $\mathbb{C}$}

Recall that the Lie superalgebra $\mathfrak{g}$ without proper ideals and of dimension $>1$ is said to be simple. Examples: Serial Lie superalgebras $\mathfrak{s l}(m \mid n)$ for $m>n \geq 1, \mathfrak{p s l}(n \mid n):=\mathfrak{s l}(n \mid n) / \mathfrak{c}$ for $n>1, \mathfrak{o s p}(m \mid 2 n)$ for $m n \neq 0$, and $\mathfrak{s p e}(n)$ for $n>2$; and the exceptional Lie superalgebras: $\mathfrak{a} \mathfrak{g}(2), \mathfrak{a} \mathfrak{b}(3)$, and $\mathfrak{o s p}(4 \mid 2 ; \alpha)$ for $\alpha \neq 0,-1$.

\section{What the Lie superalgebra in characteristic 2 is (from [34])}

Let us give a naive definition of a Lie superalgebra for $p=2$. We define it as a superspace $\mathfrak{g}=\mathfrak{g}_{\overline{0}} \oplus \mathfrak{g}_{\overline{1}}$ such that $\mathfrak{g}_{0}$ is a Lie algebra, $\mathfrak{g}_{\overline{1}}$ is an $\mathfrak{g}_{\overline{0}}$-module (made into the two-sided one by anti-symmetry, but if $p=2$, it is the same) and on $\mathfrak{g}_{\overline{1}}$ a squaring (roughly speaking, the halved bracket) is defined as a map

$$
\begin{aligned}
& x \mapsto x^{2} \text { such that }(a x)^{2}=a^{2} x^{2} \text { for any } x \in \mathfrak{g}_{\overline{1}} \text { and } a \in \mathbb{K}, \text { and } \\
& (x+y)^{2}-x^{2}-y^{2} \text { is a bilinear form on } \mathfrak{g}_{\overline{1}} \text { with values in } \mathfrak{g}_{\overline{0}} .
\end{aligned}
$$

(We use a minus sign, so the definition also works for $p \neq 2$.) The origin of this operation is as follows: If char $\mathbb{K} \neq 2$, then for any Lie superalgebra $\mathfrak{g}$ and any odd element $x \in \mathfrak{g}_{\overline{1}}$, we have $x^{2}=\frac{1}{2}[x, x] \in \mathfrak{g}_{\overline{0}}$. If $p=2$, we define $x^{2}$ first, and then define the bracket of odd elements to be (this equation is valid for $p \neq 2$ as well):

$$
[x, y]:=(x+y)^{2}-x^{2}-y^{2} .
$$

We also assume, as usual, that

if $x, y \in \mathfrak{g}_{\overline{0}}$, then $[x, y]$ is the bracket on the Lie algebra;

if $x \in \mathfrak{g}_{\overline{0}}$ and $y \in \mathfrak{g}_{\overline{1}}$, then $[x, y]:=l_{x}(y)=-[y, x]=-r_{x}(y)$, where $l$

and $r$ are the left and right $\mathfrak{g}_{0}$-actions on $\mathfrak{g}_{1}$, respectively.

The Jacobi identity involving odd elements has now the following form:

$$
\left[x^{2}, y\right]=[x,[x, y]] \text { for any } x \in \mathfrak{g}_{\overline{1}}, y \in \mathfrak{g} .
$$

If $\mathbb{K} \neq \mathbb{Z} / 2 \mathbb{Z}$, we can replace the condition on two odd elements by a simpler one:

$$
\left[x, x^{2}\right]=0 \quad \text { for any } x \in \mathfrak{g}_{1} .
$$

Because of the squaring, the definition of derived algebras should be modified. For any Lie superalgebra $\mathfrak{g}$, set $\mathfrak{g}^{(0)}:=\mathfrak{g}$ and

$$
\mathfrak{g}^{(1)}:=[\mathfrak{g}, \mathfrak{g}]+\operatorname{Span}\left\{g^{2} \mid g \in \mathfrak{g}_{\overline{1}}\right\}, \quad \mathfrak{g}^{(i+1)}:=\left[\mathfrak{g}^{(i)}, \mathfrak{g}^{(i)}\right]+\operatorname{Span}\left\{g^{2} \mid g \in\left(\mathfrak{g}^{(i)}\right)_{\overline{1}}\right\} .
$$

\subsection{Examples: Lie superalgebras preserving non-degenerate forms [34]}

Lebedev investigated various types of equivalence of bilinear forms for $p=2$, see [34]; we just recall the verdict and say that two (anti)-symmetric bilinear forms $B$ and $B^{\prime}$ on a superspace $V$ are equivalent if there is an even non-degenerate linear map $M: V \rightarrow V$ such that

$$
B^{\prime}(x, y)=B(M x, M y) \text { for all } x, y \in V .
$$

We fix some basis in $V$ and identify a given bilinear form with its Gram matrix in this basis; let us also identify any linear operator on $V$ with its matrix. Then two bilinear forms (rather supermatrices) are equivalent if there is an even invertible matrix $M$ such that

$$
B^{\prime}=M B M^{T}, \quad \text { where } T \text { is for transposition. }
$$


We often use the following matrices

$$
J_{2 n}=\left(\begin{array}{cc}
0 & 1_{n} \\
-1_{n} & 0
\end{array}\right), \quad \Pi_{n}= \begin{cases}\left(\begin{array}{cc}
0 & 1_{k} \\
1_{k} & 0
\end{array}\right) & \text { if } n=2 k \\
\left(\begin{array}{ccc}
0 & 0 & 1_{k} \\
0 & 1 & 0 \\
1_{k} & 0 & 0
\end{array}\right) & \text { if } n=2 k+1 .\end{cases}
$$

Let $J_{n \mid n}$ and $\Pi_{n \mid n}$ be the same as $J_{2 n}$ and $\Pi_{2 n}$ but considered as supermatrices.

Lebedev proved that, with respect to the above natural ${ }^{3}$ equivalence of forms (3.7), every even symmetric non-degenerate form on a superspace of dimension $n_{\overline{0}} \mid n_{\overline{1}}$ over a perfect field of characteristic 2 is equivalent to a form of the shape (here: $i=\overline{0}$ or $\overline{1}$ and each $n_{i}$ may equal to 0$)$

$$
B=\left(\begin{array}{cc}
B_{\overline{0}} & 0 \\
0 & B_{\overline{1}}
\end{array}\right), \quad \text { where } B_{i}= \begin{cases}1_{n_{i}} & \text { if } n_{i} \text { is odd } \\
\text { either } 1_{n_{i}} \text { or } \Pi_{n_{i}} & \text { if } n_{i} \text { is even }\end{cases}
$$

In other words, the bilinear forms with matrices $1_{n}$ and $\Pi_{n}$ are equivalent if $n$ is odd and nonequivalent if $n$ is even; $\operatorname{antidiag}(1, \ldots, 1) \sim \Pi_{n}$ for any $n$. The precise statement is as follows:

3.1.1. Theorem. Let $\mathbb{K}$ be a perfect field of characteristic 2 . Let $V$ be an $n$-dimensional space over $\mathbb{K}$.

1) For $n$ odd, there is only one equivalence class of non-degenerate symmetric bilinear forms on $V$.

2) For $n$ even, there are two equivalence classes of non-degenerate symmetric bilinear forms, one - with at least one non-zero element on the main diagonal - contains $1_{n}$ and the other onewith only $0 s$ on the main diagonal - contains $S_{n}:=\operatorname{antidiag}(1, \ldots, 1)$ and $\Pi_{n}$.

The Lie superalgebra preserving $B$ - by analogy with the orthosymplectic Lie superalgebras $\mathfrak{o s p}$ in characteristic 0 we call it ortho-orthogonal and denote $\mathfrak{o o}_{B}\left(n_{\overline{0}} \mid n_{\overline{1}}\right)$ - is spanned by the supermatrices which in the standard format are of the form

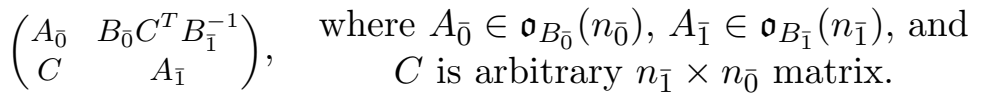

Since, as is easy to see,

$$
\mathfrak{o o}_{\Pi I}\left(n_{\overline{0}} \mid n_{\overline{1}}\right) \simeq \mathfrak{o o}_{\mathrm{I \Pi}}\left(n_{\overline{1}} \mid n_{\overline{0}}\right),
$$

we do not have to consider the Lie superalgebra $\mathfrak{o o}_{\Pi I}\left(n_{\overline{0}} \mid n_{\overline{1}}\right)$ separately unless we study Cartan prolongations - the case where the difference between these two incarnations of the same algebra is vital.

For an odd symmetric form $B$ on a superspace of dimension $\left(n_{\overline{0}} \mid n_{\overline{1}}\right)$ over a field of characteristic 2 to be non-degenerate, we need $n_{\overline{0}}=n_{\overline{1}}$, and every such form $B$ is equivalent to $\Pi_{k \mid k}$, where $k=n_{\overline{0}}=n_{\overline{1}}$. This form is preserved by linear transformations with supermatrices in the standard format of the shape

$$
\left(\begin{array}{cc}
A & C \\
D & A^{T}
\end{array}\right), \quad \text { where } A \in \mathfrak{g l}(k), C \text { and } D \text { are symmetric } k \times k \text { matrices. }
$$

The Lie superalgebra $\mathfrak{p e}(k)$ of supermatrices (3.9) will be referred to as periplectic, as A. Weil suggested, and denoted by $\mathfrak{p e}_{B}(k)$ or just $\mathfrak{p} \mathfrak{e}(k)$. (Notice that the matrix realization of $\mathfrak{p} \mathfrak{e}_{B}(k)$

\footnotetext{
${ }^{3}$ It is interesting and unexpected that for non-symmetric bilinear forms, another equivalence is more natural.
} 
over $\mathbb{C}$ or $\mathbb{R}$ is different: its set of roots is not symmetric relative the change of roots "positive $\longleftrightarrow$ negative".)

\section{The fact that two bilinear forms are inequivalent does not, generally, imply that the Lie (super)algebras that preserve them are not isomorphic.}

In [34], Lebedev proved that for the non-degenerate symmetric forms, this implication (3.10) is, however, true (except for $\mathfrak{o o}_{I \Pi}\left(n_{\overline{0}} \mid n_{\overline{1}}\right) \simeq \mathfrak{o o}_{\Pi I}\left(n_{\overline{1}} \mid n_{\overline{0}}\right)$ and $\left.\mathfrak{o o}_{\Pi \Pi}^{(1)}(6 \mid 2) \simeq \mathfrak{p e}^{(1)}(4)\right)$ and described the distinct types of Lie (super)algebras preserving non-degenerate forms. In what follows, we describe which of these Lie (super)algebras (or their derived ones) are simple, and which of them (or their central extensions) "have Cartan matrix". But first, we recall what does the term in quotation marks mean.

\section{What $\mathfrak{g}(A)$ is}

\subsection{Warning: certain of $\mathfrak{s l}$ 's and all $\mathfrak{p s l}$ 's have no Cartan matrix. Which of their relatives have Cartan matrices}

For the most reasonable definition of Lie algebra with Cartan matrix over $\mathbb{C}$, see [28]. The same definition applies, practically literally, to Lie superalgebras and to modular Lie algebras and to modular Lie superalgebras. However, the usual sloppy practice is to attribute Cartan matrices to many of those (usually simple) modular Lie algebras and (modular or not) Lie superalgebras which, strictly speaking, have no Cartan matrix!

Although it may look strange for the reader with non-super experience over $\mathbb{C}$, neither the simple modular Lie algebra $\mathfrak{p s l}(p k)$, nor the simple modular Lie superalgebra $\mathfrak{p s l}(a \mid p k+a)$, nor - in characteristic 0 - the simple Lie superalgebra $\mathfrak{p s l}(a \mid a)$ possesses a Cartan matrix. Their central extensions $-\mathfrak{s l}(p k)$, the modular Lie superalgebra $\mathfrak{s l}(a \mid p k+a)$, and - in characteristic $0-$ the Lie superalgebra $\mathfrak{s l}(a \mid a)$ - do not have Cartan matrix, either.

Their relatives possessing a Cartan matrix are, respectively, $\mathfrak{g l}(p k), \mathfrak{g l}(a \mid p k+a)$, and $\mathfrak{g l}(a \mid a)$, and for the "extra" (from the point of view of $\mathfrak{s l}$ or $\mathfrak{p s l}$ ) grading operator (such operators are denoted in what follows $d_{i}$, to distinguish them from the "inner" grading operators $h_{i}$ ) we take $E_{1,1}$.

Since often all the Lie (super)algebras involved (the simple one, its central extension, the derivation algebras thereof) are needed (and only representatives of one of the latter types of Lie (super)algebras are of the form $\mathfrak{g}(A)$ ), it is important to have (preferably short and easy to remember) notation for each of them.

For the Lie algebras that preserve a tensor (bilinear form or a volume element) we retain the same notation in all characteristic; but the (super)dimension of various incarnations of the algebras with the same name may differ as characteristic changes; simplicity may also be somewhat spoilt.

For the Lie (super)algebras which are easiest to be determined by their Cartan matrix (the Elduque Supermagic Square is of little help here), we have:

for $p=3: \mathfrak{e}(6)$ is of dimension 79 , its derived $\mathfrak{e}(6)^{(1)}$ is of dimension 78 , whereas its "simple core" is $\mathfrak{e}(6)^{(1)} / \mathfrak{c}$ of dimension 77 ;

$\mathfrak{g}(2)$ is not simple (moreover, its CM is decomposable); its "simple core" is isomorphic to $\mathfrak{p s t}(3)$;

for $p=2: \mathfrak{e}(7)$ is of dimension 134 , its derived $\mathfrak{e}(7)^{(1)}$ is of dimension 133 , whereas its "simple core" is $\mathfrak{e}(7)^{(1)} / \mathfrak{c}$ of dimension 132 ; 
$\mathfrak{g}(2)$ constructed from its CM reduced modulo 2 is now isomorphic to $\mathfrak{g l}(4)$; it is not simple, its "simple core" is isomorphic to $\mathfrak{p s l}(4)$;

the orthogonal Lie algebras and their super analogs are considered in detail later.

In our examples, the notation $D / d \mid B$ means that $\operatorname{sdim} \mathfrak{g}(A)=D \mid B$ whereas $\operatorname{sdim} \mathfrak{g}(A)^{(i)} / \mathfrak{c}=$ $d \mid B$, where

$$
d=D-2(\operatorname{size}(A)-\operatorname{rk}(A)) \quad \text { and } \quad i=\operatorname{size}(A)-\operatorname{rk}(A)=\operatorname{dim} \mathfrak{c} .
$$

\subsection{Generalities}

Let us start with the construction of a CM Lie (super)algebra. Let $A=\left(A_{i j}\right)$ be an $n \times n$-matrix. Let rk $A=n-l$. It means that there exists an $l \times n$-matrix $T=\left(T_{i j}\right)$ such that

a) the rows of $T$ are linearly independent;

b) $T A=0$ (or, more precisely, "zero $l \times n$-matrix").

Indeed, if $\operatorname{rk} A^{T}=\operatorname{rk} A=n-l$, then there exist $l$ linearly independent vectors $v_{i}$ such that $A^{T} v_{i}=0$; set

$$
T_{i j}=\left(v_{i}\right)_{j}
$$

Let the elements $e_{i}^{ \pm}$and $h_{i}$, where $i=1, \ldots, n$, generate a Lie superalgebra denoted $\tilde{\mathfrak{g}}(A, I)$, where $I=\left(p_{1}, \ldots p_{n}\right) \in(\mathbb{Z} / 2)^{n}$ is a collection of parities $\left(p\left(e_{i}^{ \pm}\right)=p_{i}\right)$, free except for the relations

$$
\left[e_{i}^{+}, e_{j}^{-}\right]=\delta_{i j} h_{i} ; \quad\left[h_{j}, e_{j}^{ \pm}\right]= \pm A_{i j} e_{j}^{ \pm} \quad \text { and } \quad\left[h_{i}, h_{j}\right]=0 \quad \text { for any } i, j .
$$

Let Lie (super)algebras with Cartan matrix $\mathfrak{g}(A, I)$ be the quotient of $\tilde{\mathfrak{g}}(A, I)$ modulo the ideal we explicitly described in $[3,4,2]$.

By abuse of notation we retain the notations $e_{j}^{ \pm}$and $h_{j}$ - the elements of $\tilde{\mathfrak{g}}(A, I)$ - for their images in $\mathfrak{g}(A, I)$ and $\mathfrak{g}^{(i)}(A, I)$.

The additional to (4.3) relations that turn $\tilde{\mathfrak{g}}(A, I)$ into $\mathfrak{g}(A, I)$ are of the form $R_{i}=0$ whose left sides are implicitly described, for the general Cartan matrix with entries in $\mathbb{K}$, as

the $R_{i}$ that generate the ideal $\mathfrak{r}$ maximal among the ideals of $\tilde{\mathfrak{g}}(A, I)$

whose intersection with the span of the above $h_{i}$ and the $d_{j}$ described

in equation (4.8) is zero.

Set

$$
c_{i}=\sum_{j=1}^{n} T_{i j} h_{j}, \quad \text { where } i=1, \ldots, l .
$$

Then, from the properties of the matrix $T$, we deduce that

a) the elements $c_{i}$ are linearly independent;

b) the elements $c_{i}$ are central, because

$$
\left[c_{i}, e_{j}^{ \pm}\right]= \pm\left(\sum_{k=1}^{n} T_{i k} A_{k j}\right) e_{j}^{ \pm}= \pm(T A)_{i j} e_{j}^{ \pm} .
$$

The existence of central elements means that the linear span of all the roots is of dimension $n-l$ only. (This can be explained even without central elements: The weights can be considered as column-vectors whose $i$-th coordinates are the corresponding eigenvalues of $\operatorname{ad}_{h_{i}}$. The weight 
of $e_{i}$ is, therefore, the $i$-th column of $A$. Since $\operatorname{rk} A=n-l$, the linear span of all columns of $A$ is $(n-l)$-dimensional just by definition of the rank. Since any root is an (integer) linear combination of the weights of the $e_{i}$, the linear span of all roots is $(n-l)$-dimensional.)

This means that some elements which we would like to see having different (even opposite if $p=2$ ) weights have, actually, identical weights. To fix this, we do the following: Let $B$ be an arbitrary $l \times n$-matrix such that

$$
\text { the }(n+l) \times n \text {-matrix }\left(\begin{array}{l}
A \\
B
\end{array}\right) \text { has rank } n \text {. }
$$

Let us add to the algebra $\mathfrak{g}=\tilde{\mathfrak{g}}(A, I)$ (and hence $\mathfrak{g}(A, I)$ ) the grading elements $d_{i}$, where $i=1, \ldots, l$, subject to the following relations:

$$
\left[d_{i}, e_{j}^{ \pm}\right]= \pm B_{i j} e_{j} ; \quad\left[d_{i}, d_{j}\right]=0 ; \quad\left[d_{i}, h_{j}\right]=0
$$

(the last two relations mean that the $d_{i}$ lie in the Cartan subalgebra, and even in the maximal torus which will be denoted by $\mathfrak{h})$.

Note that these $d_{i}$ are outer derivations of $\mathfrak{g}(A, I)^{(1)}$, i.e., they can not be obtained as linear combinations of brackets of the elements of $\mathfrak{g}(A, I)$ (i.e., the $d_{i}$ do not lie in $\left.\mathfrak{g}(A, I)^{(1)}\right)$.

\subsection{Roots and weights}

In this subsection, $\mathfrak{g}$ denotes one of the algebras $\mathfrak{g}(A, I)$ or $\tilde{\mathfrak{g}}(A, I)$.

Let $\mathfrak{h}$ be the span of the $h_{i}$ and the $d_{j}$. The elements of $\mathfrak{h}^{*}$ are called weights. For a given weight $\alpha$, the weight subspace of a given $\mathfrak{g}$-module $V$ is defined as

$$
V_{\alpha}=\left\{x \in V \mid \text { an integer } N>0 \text { exists such that }\left(\alpha(h)-\operatorname{ad}_{h}\right)^{N} x=0 \text { for any } h \in \mathfrak{h}\right\} .
$$

Any non-zero element $x \in V$ is said to be of weight $\alpha$. For the roots, which are particular cases of weights if $p=0$, the above definition is inconvenient because it does not lead to the modular analog of the following useful statement.

4.3.1. Statement ([28]). Over $\mathbb{C}$, the space of any Lie algebra $\mathfrak{g}$ can be represented as a direct sum of subspaces

$$
\mathfrak{g}=\bigoplus_{\alpha \in \mathfrak{h}^{*}} \mathfrak{g}_{\alpha}
$$

Note that if $p=2$, it might happen that $\mathfrak{h} \subsetneq \mathfrak{g}_{0}$. (For example, all weights of the form $2 \alpha$ over $\mathbb{C}$ become 0 over $\mathbb{K}$.)

To salvage the formulation of Statement in the modular case with minimal changes, at least for the Lie (super)algebras $\mathfrak{g}$ with Cartan matrix - and only this case we will have in mind speaking of roots, we decree that the elements $e_{i}^{ \pm}$with the same superscript (either + or - ) correspond to linearly independent roots $\alpha_{i}$, and any root $\alpha$ such that $\mathfrak{g}_{\alpha} \neq 0$ lies in the $\mathbb{Z}$-span of $\left\{\alpha_{1}, \ldots, \alpha_{n}\right\}$, i.e.,

$$
\mathfrak{g}=\bigoplus_{\alpha \in \mathbb{Z}\left\{\alpha_{1}, \ldots, \alpha_{n}\right\}} \mathfrak{g}_{\alpha}
$$

Thus, $\mathfrak{g}$ has a $\mathbb{R}^{n}$-grading such that $e_{i}^{ \pm}$has grade $(0, \ldots, 0, \pm 1,0, \ldots, 0)$, where \pm 1 stands in the $i$-th slot (this can also be considered as $\mathbb{Z}^{n}$-grading, but we use $\mathbb{R}^{n}$ for simplicity of formulations). If $p=0$, this grading is equivalent to the weight grading of $\mathfrak{g}$. If $p>0$, these gradings may be inequivalent; in particular, if $p=2$, then the elements $e_{i}^{+}$and $e_{i}^{-}$have the same weight. (That is why in what follows we consider roots as elements of $\mathbb{R}^{n}$, not as weights.) 
Any non-zero element $\alpha \in \mathbb{R}^{n}$ is called a root if the corresponding eigenspace of grade $\alpha$ (which we denote $\mathfrak{g}_{\alpha}$ by abuse of notation) is non-zero. The set $R$ of all roots is called the root system of $\mathfrak{g}$.

Clearly, the subspaces $\mathfrak{g}_{\alpha}$ are purely even or purely odd, and the corresponding roots are said to be even or odd.

\subsection{Systems of simple and positive roots}

In this subsection, $\mathfrak{g}=\mathfrak{g}(A, I)$, and $R$ is the root system of $\mathfrak{g}$.

For any subset $B=\left\{\sigma_{1}, \ldots, \sigma_{m}\right\} \subset R$, we set (we denote by $\mathbb{Z}_{+}$the set of non-negative integers):

$$
R_{B}^{ \pm}=\left\{\alpha \in R \mid \alpha= \pm \sum n_{i} \sigma_{i}, \text { where } n_{i} \in \mathbb{Z}_{+}\right\}
$$

The set $B$ is called a system of simple roots of $R$ (or $\mathfrak{g}$ ) if $\sigma_{1}, \ldots, \sigma_{m}$ are linearly independent and $R=R_{B}^{+} \cup R_{B}^{-}$. Note that $R$ contains basis coordinate vectors, and therefore spans $\mathbb{R}^{n}$; thus, any system of simple roots contains exactly $n$ elements.

A subset $R^{+} \subset R$ is called a system of positive roots of $R$ (or $\mathfrak{g}$ ) if there exists $x \in \mathbb{R}^{n}$ such that

$$
(\alpha, x) \in \mathbb{R} \backslash\{0\} \text { for all } \alpha \in R, \quad R^{+}=\{\alpha \in R \mid(\alpha, x)>0\} .
$$

(Here $(\cdot, \cdot)$ is the standard Euclidean inner product in $\mathbb{R}^{n}$ ). Since $R$ is a finite (or, at least, countable if $\operatorname{dim} \mathfrak{g}(A)=\infty)$ set, so the set

$$
\left\{y \in \mathbb{R}^{n} \mid \text { there exists } \alpha \in R \text { such that }(\alpha, y)=0\right\}
$$

is a finite/countable union of $(n-1)$-dimensional subspaces in $\mathbb{R}^{n}$, so it has zero measure. So for almost every $x$, condition (4.11) holds.

By construction, any system $B$ of simple roots is contained in exactly one system of positive roots, which is precisely $R_{B}^{+}$.

4.4.1. Statement. Any finite system $R^{+}$of positive roots of $\mathfrak{g}$ contains exactly one system of simple roots. This system consists of all the positive roots (i.e., elements of $R^{+}$) that can not be represented as a sum of two positive roots.

We can not give an a priori proof of the fact that each set of all positive roots each of which is not a sum of two other positive roots consists of linearly independent elements. This is, however, true for finite dimensional Lie algebras and Lie superalgebras of the form $\mathfrak{g}(A)$ if $p \neq 2$.

\subsection{Normalization convention}

Clearly,

the rescaling $e_{i}^{ \pm} \mapsto \sqrt{\lambda_{i}} e_{i}^{ \pm}$, sends $A$ to $A^{\prime}:=\operatorname{diag}\left(\lambda_{1}, \ldots, \lambda_{n}\right) \cdot A$.

Two pairs $(A, I)$ and $\left(A^{\prime}, I^{\prime}\right)$ are said to be equivalent if $\left(A^{\prime}, I^{\prime}\right)$ is obtained from $(A, I)$ by a composition of a permutation of parities and a rescaling $A^{\prime}=\operatorname{diag}\left(\lambda_{1}, \ldots, \lambda_{n}\right) \cdot A$, where $\lambda_{1} \cdots \lambda_{n} \neq 0$. Clearly, equivalent pairs determine isomorphic Lie superalgebras.

The rescaling affects only the matrix $A_{B}$, not the set of parities $I_{B}$. The Cartan matrix $A$ is said to be normalized if

$$
A_{j j}=0 \text { or } 1 \text {, or } 2 \text {. }
$$


We let $A_{j j}=2$ only if $i_{j}=\overline{0}$; in order to distinguish between the cases where $i_{j}=\overline{0}$ and $i_{j}=\overline{1}$, we write $A_{j j}=\overline{0}$ or $\overline{1}$, instead of 0 or 1 , if $i_{j}=\overline{0}$. We will only consider normalized Cartan matrices; for them, we do not have to describe $I$.

The row with a 0 or $\overline{0}$ on the main diagonal can be multiplied by any nonzero factor; usually (not only in this paper) we multiply the rows so as to make $A_{B}$ symmetric, if possible.

\subsection{Equivalent systems of simple roots}

Let $B=\left\{\alpha_{1}, \ldots, \alpha_{n}\right\}$ be a system of simple roots. Choose non-zero elements $e_{i}^{ \pm}$in the 1dimensional (by definition) superspaces $\mathfrak{g}_{ \pm \alpha_{i}}$; set $h_{i}=\left[e_{i}^{+}, e_{i}^{-}\right]$, let $A_{B}=\left(A_{i j}\right)$, where the entries $A_{i j}$ are recovered from relations (4.3), and let $I_{B}=\left\{p\left(e_{1}\right), \cdots, p\left(e_{n}\right)\right\}$. Lemma 7.3 .2 claims that all the pairs $\left(A_{B}, I_{B}\right)$ are equivalent to each other.

Two systems of simple roots $B_{1}$ and $B_{2}$ are said to be equivalent if the pairs $\left(A_{B_{1}}, I_{B_{1}}\right)$ and $\left(A_{B_{2}}, I_{B_{2}}\right)$ are equivalent.

It would be nice to find a convenient way to fix some distinguished pair $\left(A_{B}, I_{B}\right)$ in the equivalence class. For the role of the "best" (first among equals) order of indices we propose the one that minimizes the value

$$
\underset{i, j \in\{1, \ldots, n\} \text { such that }\left(A_{B}\right)_{i j} \neq 0}{\max }|i-j|
$$

(i.e., gather the non-zero entries of $A$ as close to the main diagonal as possible). Observe that this numbering differs from the one that Bourbaki use for the $\mathfrak{e}$ type Lie algebras.

\subsubsection{Chevalley generators and Chevalley bases}

We often denote the set of generators corresponding to a normalized matrix by $X_{1}^{ \pm}, \ldots, X_{n}^{ \pm}$ instead of $e_{1}^{ \pm}, \ldots, e_{n}^{ \pm}$; and call them, together with the elements $H_{i}:=\left[X_{i}^{+}, X_{i}^{-}\right]$, and the derivatives $d_{j}$ added for convenience for all $i$ and $j$, the Chevalley generators.

For $p=0$ and normalized Cartan matrices of simple finite dimensional Lie algebras, there exists only one (up to signs) basis containing $X_{i}^{ \pm}$and $H_{i}$ in which $A_{i i}=2$ for all $i$ and all structure constants are integer, cf. [51]. Such a basis is called the Chevalley basis.

Observe that, having normalized the Cartan matrix of $\mathfrak{o}(2 n+1)$ so that $A_{i i}=2$ for all $i \neq n$ but $A_{n n}=1$, we get another basis with integer structure constants. We think that this basis also qualifies to be called Chevalley basis; for Lie superalgebras, and if $p=2$, such normalization is a must.

Conjecture. If $p>2$, then for finite dimensional Lie (super)algebras with indecomposable Cartan matrices normalized as in (4.13), there also exists only one (up to signs) analog of the Chevalley basis.

We had no idea how to describe analogs of Chevalley bases for $p=2$ until recently; clearly, the methods of the recent paper [12] should solve the problem.

\section{$5 \quad$ Restricted Lie superalgebras}

Let $\mathfrak{g}$ be a Lie algebra of characteristic $p>0$. Then, for every $x \in \mathfrak{g}$, the operator $\operatorname{ad}_{x}^{p}$ is a derivation of $\mathfrak{g}$. If it is an inner derivation for every $x \in \mathfrak{g}$, i.e., if $\operatorname{ad}_{x}^{p}=\operatorname{ad}_{x^{[p]}}$ for some element denoted $x^{[p]}$, then the corresponding map

$$
[p]: x \mapsto x^{[p]}
$$

is called a $p$-structure on $\mathfrak{g}$, and the Lie algebra $\mathfrak{g}$ endowed with a $p$-structure is called a restricted Lie algebra. If $\mathfrak{g}$ has no center, then $\mathfrak{g}$ can have not more than one $p$-structure. The Lie algebra 
$\mathfrak{g l}(n)$ possesses a $p$-structure, unique up to the contribution of the center; this $p$-structure is used in the next definition.

The notion of a p-representation is naturally defined as a linear map $\rho: \mathfrak{g} \longrightarrow \mathfrak{g l}(V)$ such that $\left.\rho\left(x^{[p]}\right)=(\rho(x))^{[p]}\right)$; in this case $V$ is said to be a $p$-module.

Passing to superalgebras, we see that, for any odd $D \in \mathfrak{d e r} A$, we have

$$
D^{2 n}([a, b])=\sum\left(\begin{array}{c}
n \\
l
\end{array}\right)\left[D^{2 l}(a), D^{2 n-2 l}(b)\right] \text { for any } a, b \in A .
$$

So, if char $\mathbb{K}=p$, then $D^{2 p}$ is always an even derivation for any odd $D \in \mathfrak{d e r} A$. Now, let $\mathfrak{g}$ be a Lie superalgebra of characteristic $p>0$. Then

for every $x \in \mathfrak{g}_{\overline{0}}$, the operator $\operatorname{ad}_{x}^{p}$ is a derivation of $\mathfrak{g}$, i.e., $\mathfrak{g}_{\overline{0}}$-action on

$\mathfrak{g}_{\overline{1}}$ is a $p$-representation;

for every $x \in \mathfrak{g}_{\overline{1}}$, the operator $\operatorname{ad}_{x}^{2 p}=\operatorname{ad}_{x^{2}}^{p}$ is a derivation of $\mathfrak{g}$.

So, if for every $x \in \mathfrak{g}_{\overline{0}}$, there is $x^{[p]} \in \mathfrak{g}_{\overline{0}}$ such that $\operatorname{ad}_{x}^{p}=\operatorname{ad}_{x[p]}$ for any $x \in \mathfrak{g}_{\overline{0}}$, then we can define $x^{[2 p]}:=\left(x^{2}\right)^{[p]}$ for any $x \in \mathfrak{g}_{\overline{1}}$. We demand that for any $x \in \mathfrak{g}_{\overline{0}}$, we have

$$
\operatorname{ad}_{x}^{p}=\operatorname{ad}_{x[p]} \text { as operators on the whole } \mathfrak{g} \text {, i.e., } \mathfrak{g}_{\overline{1}} \text { is a restricted } \mathfrak{g}_{\overline{0}} \text {-module. }
$$

Then the pair of maps

$$
[p]: \mathfrak{g}_{\overline{0}} \longrightarrow \mathfrak{g}_{\overline{0}} \quad\left(x \mapsto x^{[p]}\right) \quad \text { and } \quad[2 p]: \mathfrak{g}_{\overline{1}} \longrightarrow \mathfrak{g}_{\overline{0}} \quad\left(x \mapsto x^{[2 p]}\right)
$$

is called a $p \mid 2 p$-structure - or just $p$-structure - on $\mathfrak{g}$, and the Lie superalgebra $\mathfrak{g}$ endowed with a $p$-structure is called a restricted Lie superalgebra.

\subsection{The case where $\mathfrak{g}_{\overline{0}}$ has center}

The $p$-structure on $\mathfrak{g}_{\overline{0}}$ does not have to determine a $p \mid 2 p$-structure on $\mathfrak{g}$ : Even if the actions of $\operatorname{ad}_{x}^{p}$ and $\operatorname{ad}_{x[p]}$ coincide on $\mathfrak{g}_{0}$, they do not have to coincide on the whole of $\mathfrak{g}$. This remark affects even simple Lie superalgebras if $\mathfrak{g}_{\overline{0}}$ has center. We can not say if a $p$-structure on $\mathfrak{g}_{\overline{0}}$ defines a $p \mid 2 p$-structure on $\mathfrak{g}$ in the case of centerless $\mathfrak{g}_{\overline{0}}$ : To define it we need to have, separately, a $p$-module structure on $\mathfrak{g}_{\overline{1}}$ over $\mathfrak{g}_{\overline{0}}$.

For the case where the Lie superalgebra $\mathfrak{g}$ or even $\mathfrak{g}_{\overline{0}}$ has center, the following definition is more appropriate: $\mathfrak{g}$ is said to be restricted if

there is given the map $[p]: x \mapsto x^{[p]}$ for any $x \in \mathfrak{g}_{\overline{0}}$

such that, for any $a \in \mathbb{K}$, we have

1) $(a x)^{[p]}=a^{p} \cdot x^{[p]}$ for any $x \in \mathfrak{g}_{\overline{0}}, a \in \mathbb{K}$,

2) $(x+y)^{[p]}=x^{[p]}+y^{[p]}+\sum_{i=1}^{p-1} s_{i}(x, y)$ for any $x, y \in \mathfrak{g}_{\overline{0}}$,

where $i s_{i}(x, y)$ is the coefficient of $a^{i-1}$ in the expression of $\left(\operatorname{ad}_{a x+y}\right)^{p-1}(x)$

for an indeterminate $a$,

3) $\left[x^{[p]}, y\right]=\left(\operatorname{ad}_{x}\right)^{p}(y)$ for any $x \in \mathfrak{g}_{\overline{0}}, y \in \mathfrak{g}$.

We set

$[2 p]: x \mapsto x^{[2 p]}:=\left(x^{2}\right)^{[p]} \quad$ for any $x \in \mathfrak{g}_{\overline{1}}$ 
5.1.1. Remark. If $\mathfrak{g}$ is centerless, we do not need conditions 1) and 2) of (5.4) since they follow from 3).

\subsection{Proposition.}

1) If $p>2\left(\right.$ or $p=2$ but $A_{i i} \neq \overline{1}$ for all $\left.i\right)$ and $\mathfrak{g}(A)$ is finite-dimensional, then $\mathfrak{g}(A)$ has a $p \mid 2 p$-structure such that

$$
\left(x_{\alpha}\right)^{[p]}=0 \text { for any even } \alpha \in R \text { and } x_{\alpha} \in \mathfrak{g}_{\alpha}, \quad \mathfrak{h}^{[p]} \subset \mathfrak{h} .
$$

2) If all the entries of $A$ are elements of $\mathbb{Z} / p \mathbb{Z}$, then we can set $h_{i}^{[p]}=h_{i}$ for all $i=1, \ldots, n$.

3 ) The quotient modulo center of $\mathfrak{g}(A)$ or $\mathfrak{g}^{(1)}(A)$ always inherits the $p$-structure of $\mathfrak{g}(A)$ or $\mathfrak{g}^{(1)}(A)$ (if any) whereas $\mathfrak{g}^{(1)}(A)$ does not necessarily inherit the p-structure of $\mathfrak{g}(A)$.

Proof. For the simple Lie algebras, the $p$-structure is unique if any exists, see [27]. The same proof applies to simple Lie superalgebras and $p \mid 2 p$-structures. The explicit construction completes the proof of headings 1 ) and 2). To prove 3 ) a counterexample suffices; we leave it as an exercise to the reader to produce one.

\subsubsection{Remarks.}

1) It is not enough to define $p \mid 2 p$-structure on generators, one has to define it on a basis.

2) If $p\left(X_{i}^{ \pm}\right)=\overline{1}$, then $\left(X_{i}^{ \pm}\right)^{[p]}$ is not defined unless $p=2$ : Only $\left(X_{i}^{ \pm}\right)^{[2 p]}$ is defined.

3 ) For examples of simple Lie superalgebras without Cartan matrix but with a $p \mid 2 p$-structure, see [37]. In addition to the expected examples of the modular versions of Lie superalgebras of vector fields, and the queer analog of the $\mathfrak{g l}$ series, there are - for $p=2$ - numerous (and hitherto unexpected) queerifications, see [37].

\subsection{2 (2,4)-structure on Lie algebras}

If $p=2$, we encounter a new phenomenon first mentioned in [33]. Namely, let $\mathfrak{g}=\mathfrak{g}_{+} \oplus \mathfrak{g}_{-}$be a $\mathbb{Z} / 2$-grading of a Lie algebra. We say that $\mathfrak{g}$ has a $(2,-)$-structure, if there is a 2 -structure on $\mathfrak{g}_{+}$but not on $\mathfrak{g}$. It sometimes happens that this $(2,-)$-structure can be extended to $\mathrm{a}^{4}(2,4)$ structure, which means that

$$
\text { for any } x \in \mathfrak{g}_{-} \text {there exists an } x^{[4]} \in \mathfrak{g}_{+} \text {such that } \operatorname{ad}_{x}^{4}=\operatorname{ad}_{x^{[4]}} \text {. }
$$

For example, if indecomposable symmetrizable matrix $A$ is such that $\left(\mathfrak{g}(A)=\mathfrak{o}^{(1)}(2 n+1)\right)$

$$
A_{11}=\overline{1} ; \quad A_{i i}=\overline{0} \text { for } i>1,
$$

and the Lie algebra $\mathfrak{g}(A)$ (i.e., $\mathfrak{g}(A,(\overline{0}, \ldots, \overline{0}))$ is finite-dimensional, then $\mathfrak{g}(A)$ has no 2-structure but has a $(2,4)$-structure inherited from the Lie superalgebra $\mathfrak{g}(A,(\overline{1}, \overline{0}, \ldots, \overline{0}))$.

$(\mathbf{2}, \mathbf{4} \mid \mathbf{2})$-structure on Lie superalgebras. A generalization of the $(2,4)$-structure from Lie algebras to Lie superalgebras (such as $\mathfrak{o o}_{\mathrm{I \Pi}}^{(1)}(2 n+1 \mid 2 m)$ ) is natural: Define the $\mathbb{Z} / 2$-grading $\mathfrak{g}=\mathfrak{g}_{+} \oplus \mathfrak{g}_{-}$of a Lie superalgebra having nothing to do with the parity similarly to that of $\mathfrak{g}(A)=\mathfrak{o}^{(1)}(2 n+1)$, and define the squaring on the plus part and a $(2,4)$-structure on the minus part such that the conditions

$$
\begin{aligned}
& \operatorname{ad}_{x^{[2]}}(y)=\operatorname{ad}_{x}^{2}(y) \text { for all } x \in\left(\mathfrak{o o}_{\text {II }}^{(1)}\left(2 k_{\overline{0}}+1 \mid 2 k_{\overline{1}}\right)_{\overline{0}}\right)_{+}, \\
& \operatorname{ad}_{x^{[4]}}(y)=\operatorname{ad}_{x}^{4}(y) \text { for all } x \in\left(\mathfrak{o o}_{\mathrm{I \Pi}}^{(1)}\left(2 k_{\overline{0}}+1 \mid 2 k_{\overline{1}}\right)_{\overline{0}}\right)_{-}
\end{aligned}
$$

are satisfied for any $y \in \mathfrak{o o}_{\mathrm{I \Pi}}^{(1)}\left(2 k_{\overline{0}}+1 \mid 2 k_{\overline{1}}\right)$, not only for $y \in \mathfrak{o o}_{\mathrm{I \Pi}}^{(1)}\left(2 k_{\overline{0}}+1 \mid 2 k_{\overline{1}}\right) \overline{0}$.

\footnotetext{
${ }^{4}$ Observe a slightly different notation: $(2,4)$, not $2 \mid 4$.
} 
(2|2)-structure on Lie superalgebras. Lebedev observed that if $p=2$ and a Lie superalgebra $\mathfrak{g}$ possesses a $2 \mid 4$-structure, then the Lie algebra $F(\mathfrak{g})$ one gets from $\mathfrak{g}$ by forgetting the superstructure (this is possible since $[x, x]=2 x^{2}=0$ for any odd $x$ ) possesses a 2-structure given by

the "2" part of $2 \mid 4$-structure on the former $\mathfrak{g}_{0}$;

the squaring on $\mathfrak{g}_{\overline{1}}$;

the rule $(x+y)^{[2]}=x^{[2]}+y^{[2]}+[x, y]$ on the formerly inhomogeneous (with respect to parity) elements.

So one can say that if $p=2$, then any restricted Lie superalgebra $\mathfrak{g}$ (i.e., the one with a 2|4-structure) induces a $2 \mid 2$-structure on the Lie algebra $F(\mathfrak{g})$ which is defined even on inhomogeneous elements (unlike $p \mid 2 p$-structures defined on homogeneous elements only).

5.2.2.1. Remark. The restricted Lie superalgebra structures resemble (somehow) a hidden supersymmetry of the following well-known fact:

The product of two vector fields is not necessarily a vector field, whereas

their commutator always is a vector field.

This fact was not considered to be supersymmetric until recently: Dzhumadildaev investigated a similar phenomenon: For the general and divergence-free Lie algebras of polynomial vector fields in $n$ indeterminates over $\mathbb{C}$, he investigated for which $N=N(n)$ the anti-symmetrization of the map $D \longmapsto D^{N}$ (i.e., the expression $\left.\sum_{\sigma \in S_{N}} \operatorname{sign}(\sigma) X_{\sigma(1)} \ldots X_{\sigma(N)}\right)$ yields a vector field. For the answer for $n=2,3$ and a conjecture, see [16]. But the most remarkable is Dzhumadildaev's discovery of a hidden supersymmetry of the usual commutator described by a universal odd vector field. Dzhumadildaev deduced the above fact (5.8) from the following property of odd vector fields:

The product of two vector fields is not necessarily a vector field, whereas the square of any odd field always is a vector field.

\section{Ortho-orthogonal and periplectic Lie superalgebras}

In this section, $p=2$ and $\mathbb{K}$ is perfect. We also assume that $n_{\overline{0}}, n_{\overline{1}}>0$. Set $n:=n_{\overline{0}}+n_{\overline{1}}$.

\subsection{Non-degenerate even supersymmetric bilinear forms and ortho-orthogonal Lie superalgebras}

For $p=2$, there are, in general, four equivalence classes of inequivalent non-degenerate even supersymmetric bilinear forms on a given superspace. Any such form $B$ on a superspace $V$ of superdimension $n_{\overline{0}} \mid n_{\overline{1}}$ can be decomposed as follows:

$$
B=B_{\overline{0}} \oplus B_{\overline{1}},
$$

where $B_{\overline{0}}, B_{\overline{1}}$ are symmetric non-degenerate forms on $V_{\overline{0}}$ and $V_{\overline{1}}$, respectively. For $i=\overline{0}, \overline{1}$, the form $B_{i}$ is equivalent to $1_{n_{i}}$ if $n_{i}$ is odd, and equivalent to $1_{n_{i}}$ or $\Pi_{n_{i}}$ if $n_{i}$ is even. So every non-degenerate even symmetric bilinear form is equivalent to one of the following forms (some of them are defined not for all dimensions):

$$
\begin{array}{ll}
B_{\mathrm{II}}=1_{n_{\overline{0}}} \oplus 1_{n_{\overline{1}}} ; & B_{\mathrm{I \Pi}}=1_{n_{\overline{0}}} \oplus \Pi_{n_{\overline{1}}} \text { if } n_{\overline{1}} \text { is even; } \\
B_{\Pi \mathrm{I}}=\Pi_{n_{\overline{0}}} \oplus 1_{n_{\overline{1}}} \text { if } n_{\overline{0}} \text { is even; } & B_{\Pi \Pi}=\Pi_{n_{\overline{0}}} \oplus \Pi_{n_{\overline{1}}} \text { if } n_{\overline{0}}, n_{\overline{1}} \text { are even. }
\end{array}
$$

We denote the Lie superalgebras that preserve the respective forms by $\mathfrak{o o}_{\mathrm{II}}\left(n_{\overline{0}} \mid n_{\overline{1}}\right), \mathfrak{o o}_{\mathrm{I \Pi}}\left(n_{\overline{0}} \mid n_{\overline{1}}\right)$, $\mathfrak{o o}_{\Pi \mathrm{I}}\left(n_{\overline{0}} \mid n_{\overline{1}}\right), \mathfrak{o o}_{\Pi \Pi}\left(n_{\overline{0}} \mid n_{\overline{1}}\right)$, respectively. Now let us describe these algebras. 


\subsection{1 $\mathfrak{o o}_{\mathrm{II}}\left(n_{\overline{0}} \mid n_{\overline{1}}\right)$}

If $n:=n_{\overline{0}}+n_{\overline{1}} \geq 3$, then the Lie superalgebra $\mathfrak{o o}_{\mathrm{II}}^{(1)}\left(n_{\overline{0}} \mid n_{\overline{1}}\right)$ is simple. This Lie superalgebra has a $2 \mid 4$-structure; it has no Cartan matrix.

6.1.1.1. Remark. To prove that a given Lie (super)algebra $\mathfrak{g}$ has no Cartan matrix, we have to consider its maximal tori, and for each of them, take the corresponding root grading. Then, if the simple roots are impossible to define, or the elements of weight 0 do not commute, etc. - if any of the requirements needed to define the Lie (super)algebra with Cartan matrix is violated we are done. We skip such proofs in what follows.

\subsection{2 $\mathfrak{o o}_{\text {IП }}\left(n_{\overline{0}} \mid n_{\overline{1}}\right)\left(n_{\overline{1}}=2 k_{\overline{1}}\right)$}

The Lie superalgebra $\mathfrak{o o}_{\mathrm{I \Pi}}\left(2 k_{\overline{0}}+1 \mid 2 k_{\overline{1}}\right)$ possesses a $2 \mid 4$-structure.

The Lie superalgebra $\mathfrak{o o}_{\mathrm{I \Pi}}^{(1)}\left(n_{\overline{0}} \mid n_{\overline{1}}\right)$ is simple;

$$
\mathfrak{o o}_{\mathrm{II}}^{(1)}\left(2 k_{\overline{0}}+1 \mid 2 k_{\overline{1}}\right) \quad \text { possesses } \begin{cases}2 \mid 4 \text {-structure } & \text { if } n_{\overline{0}}=1\left(k_{\overline{0}}=0\right), \\ (2,4 \mid 2) \text {-structure } & \text { if } n_{\overline{0}}>1\left(k_{\overline{0}}>0\right) ;\end{cases}
$$

$\mathfrak{o o}_{\mathrm{II}}^{(1)}\left(n_{\overline{0}} \mid n_{\overline{1}}\right)$ has a Cartan matrix if and only if $n_{\overline{0}}$ is odd; this matrix has the following form (up to a format; all possible formats - corresponding to $*=0$ or $*=\overline{0}$ - are described in Table Section 14 below):

$$
\left(\begin{array}{cccc}
\ddots & \ddots & \ddots & \vdots \\
\ddots & * & 1 & 0 \\
\ddots & 1 & * & 1 \\
\cdots & 0 & 1 & 1
\end{array}\right) .
$$

\subsection{3 $\mathfrak{o o}_{\Pi \Pi}\left(n_{\overline{0}} \mid n_{\overline{1}}\right)\left(n_{\overline{0}}=2 k_{\overline{0}}, n_{\overline{1}}=2 k_{\overline{1}}\right)$}

If $n=n_{\overline{0}}+n_{\overline{1}} \geq 6$, then

if $k_{\overline{0}}+k_{\overline{1}}$ is odd, then the Lie superalgebra $\mathfrak{o o}_{\Pi \Pi}^{(2)}\left(n_{\overline{0}} \mid n_{\overline{1}}\right)$ is simple;

if $k_{\overline{0}}+k_{\overline{1}}$ is even, then the Lie superalgebra $\mathfrak{o o}_{\Pi \Pi}^{(2)}\left(n_{\overline{0}} \mid n_{\overline{1}}\right) / \mathbb{K} 1_{n_{\overline{0}} \mid n_{\overline{1}}}$ is simple.

Each of these simple Lie superalgebras has a 2|4-structure; it is also close to a Lie superalgebra with Cartan matrix. To describe this CM Lie superalgebra in most simple terms, we will choose a slightly different realization of $\mathfrak{o o}_{\Pi \Pi}\left(2 k_{\overline{0}} \mid 2 k_{\overline{1}}\right)$ : Let us consider it as the algebra of linear transformations that preserve the bilinear form $\Pi\left(2 k_{\overline{0}}+2 k_{\overline{1}}\right)$ in the format $k_{\overline{0}}\left|k_{\overline{1}}\right| k_{\overline{0}} \mid k_{\overline{1}}$. Then the algebra $\mathfrak{o o}_{\Pi \Pi}^{(i)}\left(2 k_{\overline{0}} \mid 2 k_{\overline{1}}\right)$ is spanned by supermatrices of format $k_{\overline{0}}\left|k_{\overline{1}}\right| k_{\overline{0}} \mid k_{\overline{1}}$ and the form

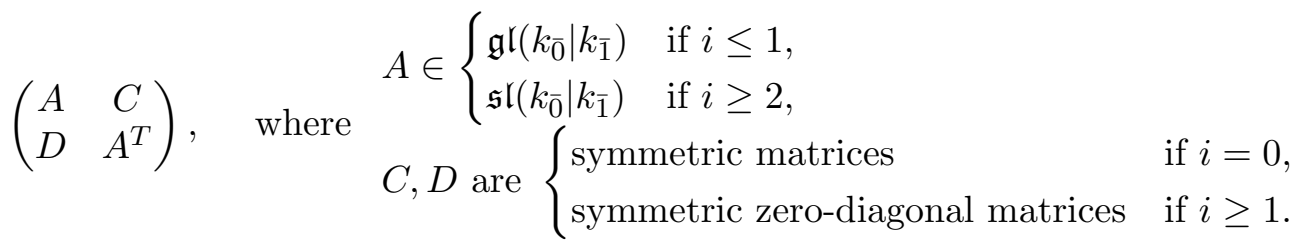

If $i \geq 1$, these derived algebras have a non-trivial central extension given by the following cocycle:

$$
F\left(\left(\begin{array}{cc}
A & C \\
D & A^{T}
\end{array}\right),\left(\begin{array}{cc}
A^{\prime} & C^{\prime} \\
D^{\prime} & A^{\prime T}
\end{array}\right)\right)=\sum_{1 \leq i<j \leq k_{\overline{0}}+k_{\overline{1}}}\left(C_{i j} D_{i j}^{\prime}+C_{i j}^{\prime} D_{i j}\right)
$$


(note that this expression resembles $\frac{1}{2} \operatorname{tr}\left(C D^{\prime}+C^{\prime} D\right)$ ). We will denote this central extension of $\mathfrak{o o}_{\Pi \Pi}^{(i)}\left(2 k_{\overline{0}} \mid 2 k_{\overline{1}}\right)$ by $\mathfrak{o o c}\left(i, 2 k_{\overline{0}} \mid 2 k_{\overline{1}}\right)$.

Let

$$
I_{0}:=\operatorname{diag}\left(1_{k_{\overline{0}} \mid k_{\overline{1}}}, 0_{k_{\overline{0}} \mid k_{\overline{1}}}\right)
$$

Then the corresponding CM Lie superalgebra is

$$
\begin{aligned}
& \mathfrak{o o c}\left(2,2 k_{\overline{0}} \mid 2 k_{\overline{1}}\right) \in \mathbb{K} I_{0} \text { if } k_{\overline{0}}+k_{\overline{1}} \text { is odd; } \\
& \mathfrak{o o c}\left(1,2 k_{\overline{0}} \mid 2 k_{\overline{1}}\right) \in \mathbb{K} I_{0} \text { if } k_{\overline{0}}+k_{\overline{1}} \text { is even. }
\end{aligned}
$$

The corresponding Cartan matrix has the form (up to format; all possible formats - corresponding to $*=0$ or $*=\overline{0}-$ are described in Table Section 14 below):

$$
\left(\begin{array}{lllll}
\ddots & \ddots & \ddots & \vdots & \vdots \\
\ddots & * & 1 & 0 & 0 \\
\ddots & 1 & * & 1 & 1 \\
\cdots & 0 & 1 & \overline{0} & 0 \\
\cdots & 0 & 1 & 0 & \overline{0}
\end{array}\right) .
$$

\subsection{The non-degenerate odd supersymmetric bilinear forms. Periplectic Lie superalgebras}

In this subsection, $m \geq 3$.

If $m$ is odd, then the Lie superalgebra $\mathfrak{p} \mathfrak{e}_{B}^{(2)}(m)$ is simple;

If $m$ is even, then the Lie superalgebra $\mathfrak{p e}_{B}^{(2)}(m) / \mathbb{K} 1_{m \mid m}$ is simple.

If we choose the form $B$ to be $\Pi_{m \mid m}$, then the algebras $\mathfrak{p} \mathfrak{e}_{B}^{(i)}(m)$ consist of matrices of the form (6.3); the only difference from $\mathfrak{o o}_{\Pi \Pi}^{(i)}$ is the format which in this case is $m \mid m$.

Each of these simple Lie superalgebras has a 2 -structure. Note that if $p \neq 2$, then the Lie superalgebra $\mathfrak{p e}_{B}(m)$ and its derived algebras are not close to CM Lie superalgebras (because, for example, their root system is not symmetric). If $p=2$ and $m \geq 3$, then they are close to CM Lie superalgebras; here we describe them.

The algebras $\mathfrak{p e}_{B}^{(i)}(m)$, where $i>0$, have non-trivial central extensions with cocycles (6.4); we denote these central extensions by $\mathfrak{p e c}(i, m)$. Let us introduce one more matrix

$$
I_{0}:=\operatorname{diag}\left(1_{m}, 0_{m}\right)
$$

Then the CM Lie superalgebras are

$$
\begin{aligned}
& \mathfrak{p e c}(2, m) \in \mathbb{K} I_{0} \text { if } m \text { is odd; } \\
& \mathfrak{p e c}(1, m) \in \mathbb{K} I_{0} \text { if } m \text { is even. }
\end{aligned}
$$

The corresponding Cartan matrix has the form (6.7); the only condition on its format is that the last two simple roots must have distinct parities. The corresponding Dynkin diagram is shown in Table Section 14; all its nodes, except for the "horns", may be both $\otimes$ or $\odot$, see (7.1). 


\subsection{Superdimensions}

The following expressions (with a + sign) are the superdimensions of the relatives of the orthoorthogonal and periplectic Lie superalgebras that possess Cartan matrices. To get the superdimensions of the simple relatives, one should replace +2 and +1 by -2 and -1 , respectively, in the two first lines and the four last ones:

$$
\begin{array}{ll}
\operatorname{dim} \mathfrak{o c}(1 ; 2 k) \in \mathbb{K} I_{0}=2 k^{2}-k \pm 2 & \text { if } k \text { is even; } \\
\operatorname{dim} \mathfrak{o c}(2 ; 2 k) \in \mathbb{K} I_{0}=2 k^{2}-k \pm 1 & \text { if } k \text { is odd; } \\
\operatorname{dim} \mathfrak{o}^{(1)}(2 k+1)=2 k^{2}+k ; & \\
\operatorname{sdim} \mathfrak{o o}^{(1)}\left(2 k_{\overline{0}}+1 \mid 2 k_{\overline{1}}\right)=2 k_{\overline{0}}^{2}+k_{\overline{0}}+2 k_{\overline{1}}^{2}+k_{\overline{1}} \mid 2 k_{\overline{1}}\left(2 k_{\overline{0}}+1\right) ; & \\
\operatorname{sdim} \mathfrak{o o c}\left(1 ; 2 k_{\overline{0}} \mid 2 k_{\overline{1}}\right) \in \mathbb{K} I_{0}=2 k_{\overline{0}}^{2}-k_{\overline{0}}+2 k_{\overline{1}}^{2}-k_{\overline{1}} \pm 2 \mid 4 k_{\overline{0}} k_{\overline{1}} & \text { if } k_{\overline{0}}+k_{\overline{1}} \text { is even; } \\
\operatorname{sdim} \mathfrak{o o c}\left(2 ; 2 k_{\overline{0}} \mid 2 k_{\overline{1}}\right) \in \mathbb{K} I_{0}=2 k_{\overline{0}}^{2}-k_{\overline{0}}+2 k_{\overline{1}}^{2}-k_{\overline{1}} \pm 1 \mid 4 k_{\overline{0}} k_{\overline{1}} & \text { if } k_{\overline{0}}+k_{\overline{1}} \text { is odd; } \\
\operatorname{sdim} \mathfrak{p e c}(1 ; m) \in \mathbb{K} I_{0}=m^{2} \pm 2 \mid m^{2}-m & \text { if } m \text { is even; } \\
\operatorname{sdim} \mathfrak{p e c}(2 ; m) \in \mathbb{K} I_{0}=m^{2} \pm 1 \mid m^{2}-m & \text { if } m \text { is odd. }
\end{array}
$$

\subsubsection{Summary: The types of Lie superalgebras preserving non-degenerate symmetric forms}

Let

$$
\widehat{\mathfrak{g}}:=\mathfrak{g} \in \mathbb{K} I_{0} .
$$

We have the following types of non-isomorphic Lie (super) algebras (except for an occasional isomorphism intermixing the types, e.g., $\left.\mathfrak{o o}_{\Pi \Pi}^{(1)}(6 \mid 2) \simeq \mathfrak{p} \mathfrak{e}^{(1)}(4)\right)$ :

\begin{tabular}{|l|l|}
\hline no relative has Cartan matrix & with Cartan matrix \\
\hline $\mathfrak{o O}_{\mathrm{II}}(2 n+1 \mid 2 m+1), \quad \mathfrak{o o}_{\mathrm{II}}(2 n+1 \mid 2 m)$ & $\left.\widehat{\mathfrak{o c}(i ; 2 n),} \mathfrak{o}^{(1)}(2 n+1) ; \widehat{\mathfrak{p e c}(i ; k}\right)$ \\
$\mathfrak{o o}_{\mathrm{II}}(2 n \mid 2 m), \quad \mathfrak{o o}_{\mathrm{I \Pi}}(2 n \mid 2 m) ; \quad \mathfrak{o}_{\mathrm{I}}(2 n) ;$ & $\mathfrak{o o v}(\widehat{i ; 2 n \mid} 2 m), \quad \mathfrak{o o}_{\mathrm{II}}^{(1)}(2 n+1 \mid 2 m)$ \\
\hline
\end{tabular}

The superdimensions are as follows (in the second and third column stand the additions to the superdimensions in the first column):

\begin{tabular}{|l|l|l|}
\hline $\operatorname{sdim} \mathfrak{o o}_{\mathrm{II}}(a \mid b)$ & $\operatorname{sdim} \mathfrak{o o}_{\mathrm{II}}^{(1)}(a \mid b)$ & $\operatorname{sdim} \mathfrak{o o}_{\mathrm{II}}^{(2)}(a \mid b)$ \\
\hline$\frac{1}{2} a(a+1)+\frac{1}{2} b(b+1) \mid a b$ & $-1 \mid 0$ & \\
\hline $\operatorname{sdim} \mathfrak{o o}_{\mathrm{I \Pi}}(a \mid b)$ & $\operatorname{sdim} \mathfrak{o o}_{\mathrm{II}}^{(1)}(a \mid b)$ & $\operatorname{sdim} \mathfrak{o o}_{\mathrm{II}}^{(2)}(a \mid b)$ \\
\hline$\frac{1}{2} a(a+1)+\frac{1}{2} b(b+1) \mid a b$ & $-a \mid 0$ & \\
\hline $\operatorname{sdim} \mathfrak{o o}_{\Pi \Pi}(a \mid b)$ & $\operatorname{sdim} \mathfrak{o o}_{\Pi \Pi}^{(1)}(a \mid b)$ & $\operatorname{sdim} \mathfrak{o o}_{\Pi \Pi}^{(2)}(a \mid b)$ \\
$\frac{1}{2} a(a+1)+\frac{1}{2} b(b+1) \mid a b$ & $-a-b \mid 0$ & $-1 \mid 0$ \\
\hline
\end{tabular}

\section{Dynkin diagrams}

A usual way to represent simple Lie algebras over $\mathbb{C}$ with integer Cartan matrices is via graphs called, in the finite dimensional case, Dynkin diagrams (DD). The Cartan matrices of certain interesting infinite dimensional simple Lie superalgebras $\mathfrak{g}$ (even over $\mathbb{C}$ ) can be non-symmetrizable or (for any $p$ in the super case and for $p>0$ in the non-super case) have entries belonging to the ground field $\mathbb{K}$. Still, it is always possible to assign an analog of the Dynkin diagram to each (modular) Lie (super)algebra (with Cartan matrix, of course) provided the edges and nodes of 
the graph (DD) are rigged with an extra information. Although these analogs of the Dynkin graphs are not uniquely recovered from the Cartan matrix (and the other way round), they give a graphic presentation of the Cartan matrices and help to observe some hidden symmetries.

Namely, the Dynkin diagram of a normalized $n \times n$ Cartan matrix $A$ is a set of $n$ nodes connected by multiple edges, perhaps endowed with an arrow, according to the usual rules [28] or their modification, most naturally formulated by Serganova: compare [47, 20] with [21]. In what follows, we recall these rules, and further improve them to fit the modular case.

\subsection{Nodes}

To every simple root there corresponds

$$
\begin{cases}\text { a node } \bigcirc & \text { if } p\left(\alpha_{i}\right)=\overline{0} \text { and } A_{i i}=2, \\ \text { a node } * & \text { if } p\left(\alpha_{i}\right)=\overline{0} \text { and } A_{i i}=\overline{1} \\ \text { a node } \odot & \text { if } p\left(\alpha_{i}\right)=\overline{1} \text { and } A_{i i}=1 \\ \text { a node } \otimes & \text { if } p\left(\alpha_{i}\right)=\overline{1} \text { and } A_{i i}=0 \\ \text { a node } \odot & \text { if } p\left(\alpha_{i}\right)=\overline{0} \text { and } A_{i i}=\overline{0}\end{cases}
$$

The Lie algebras $\mathfrak{s l}(2)$ and $\mathfrak{o}(3)^{(1)}$ with Cartan matrices $(2)$ and $(\overline{1})$, respectively, and the Lie superalgebra $\mathfrak{o s p}(1 \mid 2)$ (which is $\mathfrak{o o}_{\mathrm{I}}^{(1)}(1 \mid 2)$ if $p=2$ ) with Cartan matrix (1) are simple.

The Lie algebra with Cartan matrix $(\overline{0})$ and the Lie superalgebra with Cartan matrix (0) are solvable of $\operatorname{dim} 4$ and $\operatorname{sdim} 2 \mid 2$, respectively. Their derived algebras are Heisenberg algebra heil $(2) \simeq \mathfrak{h} \mathfrak{e i}(2 \mid 0)$ and Heisenberg superalgebra $\mathfrak{h} \mathfrak{e i}(0 \mid 2) \simeq \mathfrak{s l}(1 \mid 1)$, respectively; their (super)dimensions are 3 and $1 \mid 2$, respectively.

\subsubsection{Digression}

Let $\xi=\left(\xi_{1}, \ldots, \xi_{n}\right)$ and $\eta=\left(\eta_{1}, \ldots, \eta_{n}\right)$ be odd elements, $p=\left(p_{1}, \ldots, p_{m}\right), q=\left(q_{1}, \ldots, q_{m}\right)$ and $z$ even elements. $\mathfrak{h e i}(2 m \mid 2 n)=\operatorname{Span}(p, q, \xi, \eta, z)$, where the brackets are

$$
\left[p_{i}, q_{j}\right]=\delta_{i j} z, \quad\left[\xi_{i}, \eta_{j}\right]=\delta_{i j} z, \quad[z, \mathfrak{h} \mathfrak{e i}(2 m \mid 2 n)]=0 .
$$

In what follows we will need the Lie superalgebra $\mathfrak{h} \mathfrak{e i}(2 m \mid 2 n$ ) (for the cases where $m n=0$ ) and its only (up to the change of parity) non-trivial irreducible representation, called the Fock space, which in characteristic $p$ is $\mathbb{K}[q, \xi] /\left(q_{1}^{p}, \ldots, q_{n}^{p}\right)$ on which the elements $q_{i}$ and $\xi_{j}$ act as operators of left multiplication by $q_{i}$ and $\xi_{j}$, respectively, whereas $p_{i}$ and $\eta_{j}$ act as $h \partial_{q_{i}}$ and $h \partial_{\xi_{j}}$, where $h \in \mathbb{K} \backslash\{0\}$ can be fixed to be equal to 1 by a change of the basis.

7.1.2. Remark. A posteriori (from the classification of simple Lie superalgebras with Cartan matrix and of polynomial growth for $p=0$ ) we find out that the roots $\odot$ can only occur if $\mathfrak{g}(A, I)$ grows faster than polynomially. Thanks to classification again, if $\operatorname{dim} \mathfrak{g}<\infty$, the roots of type $\odot$ can not occur if $p>3$; whereas for $p=3$, the Brown Lie algebras are examples of $\mathfrak{g}(A)$ with a simple root of type $\odot$; for $p=2$, such roots are routine.

\subsection{Edges}

If $p=2$ and $\operatorname{dim} \mathfrak{g}(A)<\infty$, the Cartan matrices considered are symmetric. If $A_{i j}=a$, where $a \neq 0$ or 1 , then we rig the edge connecting the $i$ th and $j$ th nodes by a label $a$.

If $p>2$ and $\operatorname{dim} \mathfrak{g}(A)<\infty$, then $A$ is symmetrizable, so let us symmetrize it, i.e., consider $D A$ for an invertible diagonal matrix $D$. Then, if $(D A)_{i j}=a$, where $a \neq 0$ or -1 , we rig the edge connecting the $i$ th and $j$ th nodes by a label $a$. 
If all off-diagonal entries of $A$ belong to $\mathbb{Z} / p$ and their representatives are selected to be non-positive integers, we can draw the DD as for $p=0$, i.e., connect the $i$ th node with the $j$ th one by $\max \left(\left|A_{i j}\right|,\left|A_{j i}\right|\right)$ edges rigged with an arrow $>$ pointing from the $i$ th node to the $j$ th if $\left|A_{i j}\right|>\left|A_{j i}\right|$ or in the opposite direction if $\left|A_{i j}\right|<\left|A_{j i}\right|$.

\subsection{Reflections}

Let $R^{+}$be a system of positive roots of Lie superalgebra $\mathfrak{g}$, and let $B=\left\{\sigma_{1}, \ldots, \sigma_{n}\right\}$ be the corresponding system of simple roots with some corresponding pair $\left(A=A_{B}, I=I_{B}\right)$. Then for any $k \in\{1, \ldots, n\}$, the set $\left(R^{+} \backslash\left\{\sigma_{k}\right\}\right) \coprod\left\{-\sigma_{k}\right\}$ is a system of positive roots. This operation is called the reflection in $\sigma_{k}$; it changes the system of simple roots by the formulas

$$
r_{\sigma_{k}}\left(\sigma_{j}\right)= \begin{cases}-\sigma_{j} & \text { if } k=j, \\ \sigma_{j}+B_{k j} \sigma_{k} & \text { if } k \neq j,\end{cases}
$$

where

$$
B_{k j}= \begin{cases}-\frac{2 A_{k j}}{A_{k k}} & \text { if } i_{k}=\overline{0}, A_{k k} \neq 0, \text { and }-\frac{2 A_{k j}}{A_{k k}} \in \mathbb{Z} / p \mathbb{Z}, \\ p-1 & \text { if } i_{k}=\overline{0}, A_{k k} \neq 0 \text { and }-\frac{2 A_{k j}}{A_{k k}} \notin \mathbb{Z} / p \mathbb{Z}, \\ -\frac{A_{k j}}{A_{k k}} & \text { if } i_{k}=\overline{1}, A_{k k} \neq 0, \text { and }-\frac{A_{k j}}{A_{k k}} \in \mathbb{Z} / p \mathbb{Z}, \\ p-1 & \text { if } i_{k}=\overline{1}, A_{k k} \neq 0, \text { and }-\frac{A_{k j}}{A_{k k}} \notin \mathbb{Z} / p \mathbb{Z}, \\ 1 & \text { if } i_{k}=\overline{1}, A_{k k}=0, A_{k j} \neq 0, \\ 0 & \text { if } i_{k}=\overline{1}, A_{k k}=A_{k j}=0, \\ p-1 & \text { if } i_{k}=\overline{0}, A_{k k}=\overline{0}, A_{k j} \neq 0 \\ 0 & \text { if } i_{k}=\overline{0}, A_{k k}=\overline{0}, A_{k j}=0\end{cases}
$$

where we consider $\mathbb{Z} / p \mathbb{Z}$ as a subfield of $\mathbb{K}$.

7.3.1. Remark. In the second, fourth and penultimate cases, the matrix entries in (7.4) can, in principle, be equal to $k p-1$ for any $k \in \mathbb{N}$, and in the last case any element of $\mathbb{K}$ may occur. We may only hope at this stage that, at least for $\operatorname{dim} \mathfrak{g}<\infty$, this does not happen.

The values $-\frac{2 A_{k j}}{A_{k k}}$ and $-\frac{A_{k j}}{A_{k k}}$ are elements of $\mathbb{K}$, while the roots are elements of a vector space over $\mathbb{R}$. Therefore These expressions in the first and third cases in (7.4) should be understood as "the minimal non-negative integer congruent to $-\frac{2 A_{k j}}{A_{k k}}$ or $-\frac{A_{k j}}{A_{k k}}$, respectively". (If $\operatorname{dim} \mathfrak{g}<\infty$, these expressions are always congruent to integers.)

There is known just one exception: If $p=2$ and $A_{k k}=A_{j k}$, the expression $-\frac{2 A_{j k}}{A_{k k}}$ should be understood as 2 , not 0 .

The name "reflection" is used because in the case of (semi)simple finite-dimensional Lie algebras this action extended on the whole $R$ by linearity is a map from $R$ to $R$, and it does not depend on $R^{+}$, only on $\sigma_{k}$. This map is usually denoted by $r_{\sigma_{k}}$ or just $r_{k}$. The map $r_{\sigma_{i}}$ extended to the $\mathbb{R}$-span of $R$ is reflection in the hyperplane orthogonal to $\sigma_{i}$ relative the bilinear form dual to the Killing form.

The reflections in the even (odd) roots are referred to as even (odd) reflections. A simple root is called isotropic, if the corresponding row of the Cartan matrix has zero on the diagonal, and non-isotropic otherwise. The reflections that correspond to isotropic or non-isotropic roots will be referred to accordingly. 
If there are isotropic simple roots, the reflections $r_{\alpha}$ do not, as a rule, generate a version of the Weyl group because the product of two reflections in nodes not connected by one (perhaps, multiple) edge is not defined. These reflections just connect pair of "neighboring" systems of simple roots and there is no reason to expect that we can multiply two distinct such reflections. In the general case (of Lie superalgebras and $p>0$ ), the action of a given isotropic reflections (7.3) can not, generally, be extended to a linear map $R \longrightarrow R$. For Lie superalgebras over $\mathbb{C}$, one can extend the action of reflections by linearity to the root lattice but this extension preserves the root system only for $\mathfrak{s l}(m \mid n)$ and $\mathfrak{o s p}(2 m+1 \mid 2 n)$, cf. [48].

If $\sigma_{i}$ is an odd isotropic root, then the corresponding reflection sends one set of Chevalley generators into a new one:

$$
\tilde{X}_{i}^{ \pm}=X_{i}^{\mp} ; \quad \tilde{X}_{j}^{ \pm}= \begin{cases}{\left[X_{i}^{ \pm}, X_{j}^{ \pm}\right]} & \text {if } A_{i j} \neq 0, \overline{0} \\ X_{j}^{ \pm} & \text {otherwise. }\end{cases}
$$

\subsubsection{Lebedev's lemma}

Serganova [47] proved (for $p=0$ ) that there is always a chain of reflections connecting $B_{1}$ with some system of simple roots $B_{2}^{\prime}$ equivalent to $B_{2}$ in the sense of definition 4.6. Here is the modular version of Serganova's Lemma. Observe that Serganova's statement is not weaker: Serganova used only odd reflections.

Lemma ([37]). For any two systems of simple roots $B_{1}$ and $B_{2}$ of any finite dimensional Lie superalgebra with indecomposable Cartan matrix, there is always a chain of reflections connecting $B_{1}$ with $B_{2}$.

\section{A careful study of an example}

Now let $p=2$ and let us apply all the above to the Lie superalgebra $\mathfrak{p e}(k)$ (the situation with $\mathfrak{o}_{\Pi}(2 k)$ and $\mathfrak{o o}_{\Pi \Pi}\left(2 k_{\overline{0}} \mid 2 k_{\overline{1}}\right)$ is the same). For the Cartan matrix (all possible formats corresponding to $*=0$ or $*=\overline{0}$ - are listed in Table Section 14) we take

$$
A=\left(\begin{array}{cccc}
\ddots & \ddots & \ddots & \ddots \\
\cdots & * & 1 & 1 \\
\cdots & 1 & 0 & 0 \\
\cdots & 1 & 0 & 0
\end{array}\right)
$$

The Lie superalgebra $\mathfrak{p e}^{(i)}(k)$ consists of supermatrices of the form

$$
\left(\begin{array}{cc}
B & C \\
D & B^{T}
\end{array}\right)
$$

where

$$
\begin{array}{ll}
\text { for } i=0, \text { we have } & B \in \mathfrak{g l}(k), C, D \text { are symmetric; } \\
\text { for } i=1 \text {, we have } & B \in \mathfrak{g l}(k), C, D \text { are symmetric zero-diagonal; } \\
\text { for } i=2, \text { we have } & B \in \mathfrak{s l}(k), C, D \text { are symmetric zero-diagonal. }
\end{array}
$$

We expect (by analogy with the orthogonal Lie algebras in characteristic $\neq 2$ ) that

$$
\begin{aligned}
& e_{i}^{+}=E_{i, i+1}+E_{k+i+1, k+i} ; \quad e_{i}^{-}=E_{i+1, i}+E_{k+i, k+i+1} \\
& e_{k}^{+}=E_{k-1,2 k}+E_{k, 2 k-1} ; \quad e_{k}^{-}=E_{2 k-1, k}+E_{2 k, k-1} .
\end{aligned}
$$


Let us first consider the (simpler) case of $k$ odd. Then $\operatorname{rk} A=k-1$ since the sum of the last two rows is zero. Let us start with the simple algebra $\mathfrak{p e}^{(2)}(k)$. The Cartan subalgebra (i.e., the subalgebra of diagonal matrices) is $(k-1)$-dimensional because the elements $\left[e_{1}^{+}, e_{1}^{-}\right], \ldots,\left[e_{k-1}^{+}, e_{k-1}^{-}\right]$are linearly independent, whereas $\left[e_{k}^{+}, e_{k}^{-}\right]=\left[e_{k-1}^{+}, e_{k-1}^{-}\right]$. Thus, we should first find a non-trivial central extension, spanned by $z$ satisfying the condition

$$
z=\left[e_{k}^{+}, e_{k}^{-}\right]+\left[e_{k-1}^{+}, e_{k-1}^{-}\right] .
$$

Elucidation: The values of $e_{i}^{ \pm}$in (8.3) are what we expect them to be from their $p=0$ analogs. But from the definition of CM Lie superalgebra we see that the algebra must have a center $z$, see (8.4). Thus, the CM Lie superalgebra is not $\mathfrak{p e}^{(2)}(k)$ but is spanned by the central extension of $\mathfrak{p} \mathfrak{e}^{(2)}(k)$ plus the grading operator defined from (4.6). The extension $\mathfrak{p e c}(2, k)$ described in (6.4) satisfies this condition.

Now let us choose $B$ to be $(0, \ldots, 0,1)$. Then we need to add to the algebra a grading operator $d$ such that

$$
\begin{aligned}
& {\left[d, e_{i}^{ \pm}\right]=0 \text { for all } i=1, \ldots, k-1 ;} \\
& {\left[d, e_{k}^{ \pm}\right]=e_{k}^{ \pm} ;} \\
& d \text { commutes with all diagonal matrices. }
\end{aligned}
$$

The matrix $I_{0}=\operatorname{diag}\left(1_{k}, 0_{k}\right)$ satisfies all these conditions. Thus, the corresponding CM Lie superalgebra is

$$
\mathfrak{p e c}(2, k) \in \mathbb{K} I_{0} .
$$

Remark. Rather often we need ideals of CM Lie (super)algebras that do not contain the outer grading operator(s), cf. Section 4.1. These ideals, such as $\mathfrak{p e c}(2, k)$ or $\mathfrak{s l}(n \mid n)$, do not have Cartan matrix.

Now let us consider the case of $k$ even. Then the simple algebra is $\mathfrak{p e}{ }^{(2)}(k) /\left(\mathbb{K} 1_{2 k}\right)$. The Cartan matrix is of rank $k-2$ :

(a) the sum of the last two rows is zero;

(b) the sum of all the rows with odd numbers is zero.

The condition (8.7a) gives us the same central extension and the same grading operator an in the previous case.

To satisfy condition (8.7b), we should find a non-trivial central extension such that

$$
z=\sum_{i \text { is odd }}\left[e_{i}^{+}, e_{i}^{-}\right]
$$

(This formula follows from (4.5) and the 2nd equality in (8.7).) But we can see that, in $\mathfrak{p e}^{(2)}(k)$, we have

$$
\sum_{i \text { is odd }}\left[e_{i}^{+}, e_{i}^{-}\right]=\sum_{i \text { is odd }}\left(E_{i, i}+E_{i+1, i+1}+E_{k+i, k+i}+E_{k+i+1, k+i+1}\right)=1_{2 k} .
$$

It means that the corresponding central extension of $\mathfrak{p} \mathfrak{e}^{(2)}(k) /\left(\mathbb{K} 1_{2 k}\right)$ is just $\mathfrak{p} \mathfrak{e}^{(2)}(k)$.

Now, concerning the grading operator: Let the second row of $B$ be $(1,0, \ldots, 0)$ (the first row is, as in the previous case, $(0, \ldots, 0,1))$. Then we need a grading operator $d_{2}$ such that

$$
\begin{aligned}
& {\left[d_{2}, e_{1}^{ \pm}\right]=e_{1}^{ \pm}} \\
& {\left[d_{2}, e_{i}^{ \pm}\right]=0 \text { for all } i>1 ;} \\
& d_{2} \text { commutes with all diagonal matrices. }
\end{aligned}
$$

The matrix $d_{2}:=E_{1,1}+E_{k+1, k+1}$ satisfies these conditions. But $\mathfrak{p e}^{(2)}(k) \in \mathbb{K}\left(E_{1,1}+E_{k+1, k+1}\right)$ is just $\mathfrak{p e}^{(1)}(k)$. So, the resulting CM Lie superalgebra is

$$
\mathfrak{p e c}(1, k) \in \mathbb{K} I_{0} .
$$




\section{Main steps of our classification}

In this section we deal with Lie (super)algebras of the form $\mathfrak{g}(A)$ or their simple subquotients $\mathfrak{g}(A)^{(i)} / \mathfrak{c}$, where $i=1$ or 2 .

\subsection{Step 1: An overview of known results}

Lie algebras (nothing super). There are known the two methods of classification:

1) Over $\mathbb{C}$, Cartan [10] did not use any roots, instead he used what is nowadays called in his honor Cartan prolongations and a generalization (which he never formulated explicitly) of this procedure which we call CTS-ing (Cartan-Tanaka-Shchepochkina prolonging).

2) Nowadays, to get the shortest classification of the simple finite dimensional Lie algebras, everybody (e.g. $[8,41]$ ) uses root technique and the non-degenerate invariant symmetric bilinear form (the Killing form).

In the modular case, as well as in the super case, and in the mixture of these cases we consider here, the Killing form might be identically zero. However, if the Cartan matrix $A$ is symmetrizable (and indecomposable), on the Lie (super)algebra $\mathfrak{g}(A)$ if $\mathfrak{g}(A)$ is simple (or on $\mathfrak{g}(A)^{(i)} / \mathfrak{c}$ if $\mathfrak{g}(A)$ is not simple), there is a non-degenerate replacement of the Killing form. (Astonishingly, this replacement might sometimes be not coming from any representation, see [46]. Much earlier Kaplansky observed a similar phenomenon in the modular case and associated the non-degenerate bilinear form with a projective representation. Kaplansky pointed at this phenomenon in his wonderful preprints [31] which he modestly did not publish.)

In the modular case, and in the super case for $p=0$, this approach - to use a non-degenerate even invariant symmetric form in order to classify the simple algebras - was pursued by Kaplansky [31].

For $p>0$, Weisfeiler and Kac [54] gave a classification, but although the idea of their proof is $\overline{\mathrm{OK}}$, the paper has several gaps and vague notions (the Brown algebra br( $(3)$ was missed, whereas Brown [9] who discovered it did not write that it possesses Cartan matrix, actually two inequivalent matrices first observed by Skryabin [50, 30]; the notion of the Lie algebra with Cartan matrix nicely formulated in [28] was not properly developed at the time [54] was written; the Dynkin diagrams mentioned there were not defined at all in the modular case; the algebras $\mathfrak{g}(A)$ and $\mathfrak{g}(A)^{(i)} / \mathfrak{c}$ were sometimes identified). The case $p>3$ being completely investigated by Block, Wilson, Premet and Strade [42, 52] (see also [1]), we double-checked the cases where $p<5$. The answer of $[54] \cup[50]$ is correct.

Lie superalgebras.

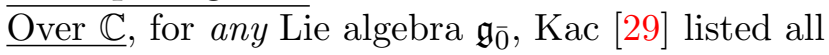

$\mathfrak{g}_{\overline{0}}$-modules $\mathfrak{g}_{\overline{1}}$ such that the Lie superalgebra $\mathfrak{g}=\mathfrak{g}_{\overline{0}} \oplus \mathfrak{g}_{\overline{1}}$ is simple.

Kaplansky [31, 22, 32], Djoković and Hochschild [15], and also Scheunert, Nahm and Rittenberg [45] had their own approaches to the problem (9.1) and solved it without gaps for various particular cases, but they did not investigate which of the simple finite dimensional Lie superalgebras possess Cartan matrix.

Kac observed that (a) some of the simple Lie superalgebras (9.1) possess analogs of Cartan matrix, (b) one Lie superalgebra may have several inequivalent Cartan matrices. His first list of inequivalent Cartan matrices (in other words, distinct $\mathbb{Z}$-gradings) for finite dimensional Lie superalgebras $\mathfrak{g}(A)$ in [29] had gaps; Serganova [47] and (by a different method and only for symmetrizable matrices) van de Leur [53] fixed the gaps and even classified Lie superalgebras of polynomial growth (for the proof in the non-symmetrizable case, announced 20 years earlier, see [26]). Kac also suggested analogs of Dynkin diagrams to graphically encode the Cartan matrices. 
Kaplansky was the first (see his newsletters in [31]) to discover the exceptional algebras $\mathfrak{a g}(2)$ and $\mathfrak{a b}(3)$ (he dubbed them $\Gamma_{2}$ and $\Gamma_{3}$, respectively) and a parametric family $\mathfrak{o s p}(4 \mid 2 ; \alpha$ ) (he dubbed it $\Gamma(A, B, C))$ ); our notations reflect the fact that $\mathfrak{a g}(2)_{\overline{0}}=\mathfrak{s l}(2) \oplus \mathfrak{g}(2)$ and $\mathfrak{a} \mathfrak{b}(3)_{\overline{0}}=$ $\mathfrak{s l}(2) \oplus \mathfrak{o}(7)\left(\mathfrak{o}(7)\right.$ is $B_{3}$ in Cartan's nomenclature). Kaplansky's description (irrelevant to us at the moment except for the fact that $A, B$ and $C$ are on equal footing) of what we now identify as $\mathfrak{o s p}(4 \mid 2 ; \alpha)$, a parametric family of deforms of $\mathfrak{o s p}(4 \mid 2)$, made an $S_{3}$-symmetry of the parameter manifest (to A. A. Kirillov, and he informed us, in 1976). Indeed, since $A+B+C=0$, and $\alpha \in \mathbb{C} \cup \infty$ is the ratio of the two remaining parameters, we get an $S_{3}$-action on the plane $A+B+C=0$ which in terms of $\alpha$ is generated by the transformations:

$$
\alpha \longmapsto-1-\alpha, \quad \alpha \longmapsto \frac{1}{\alpha} .
$$

This symmetry should have immediately sprang to mind since $\mathfrak{o} \mathfrak{p}(4 \mid 2 ; \alpha)$ is strikingly similar to $\mathfrak{w k}(3 ; a)$ found 5 years earlier, cf. (9.5), and since $S_{3} \simeq \mathrm{SL}(2 ; \mathbb{Z} / 2)$.

The following figure depicts the fundamental domains of the $S_{3}$-action. The other transformations generated by (9.2) are

$$
\alpha \longmapsto-\frac{1+\alpha}{\alpha}, \quad \alpha \longmapsto-\frac{1}{\alpha+1}, \quad \alpha \longmapsto-\frac{\alpha}{\alpha+1} .
$$

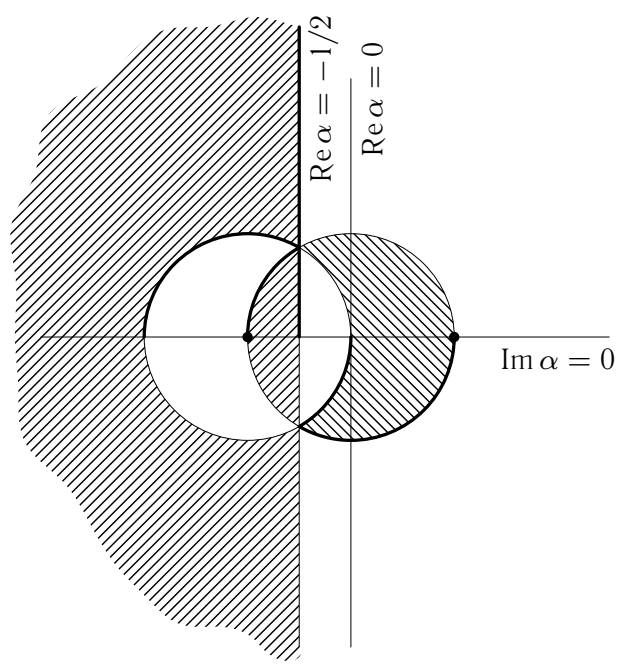

\subsubsection{Notation: On matrices with a "_" sign and other notations in the lists of inequivalent Cartan matrices}

The rectangular matrix at the beginning of each list of inequivalent Cartan matrices for each Lie superalgebra shows the result of odd reflections (the number of the row is the number of the Cartan matrix in the list below, the number of the column is the number of the root (given by small boxed number) in which the reflection is made; the cells contain the results of reflections (the number of the Cartan matrix obtained) or a "-" if the reflection is not appropriate because $A_{i i} \neq 0$. Some of the Cartan matrices thus obtained are equivalent, as indicated.

The number of the matrix $A$ such that $\mathfrak{g}(A)$ has only one odd simple root is boxed, that with all simple roots odd is underlined. The nodes are numbered by small boxed numbers; the curly lines with arrows depict odd reflections.

Recall that $\mathfrak{a g}(2)$ of sdim $=17 \mid 14$ has the following Cartan matrices 


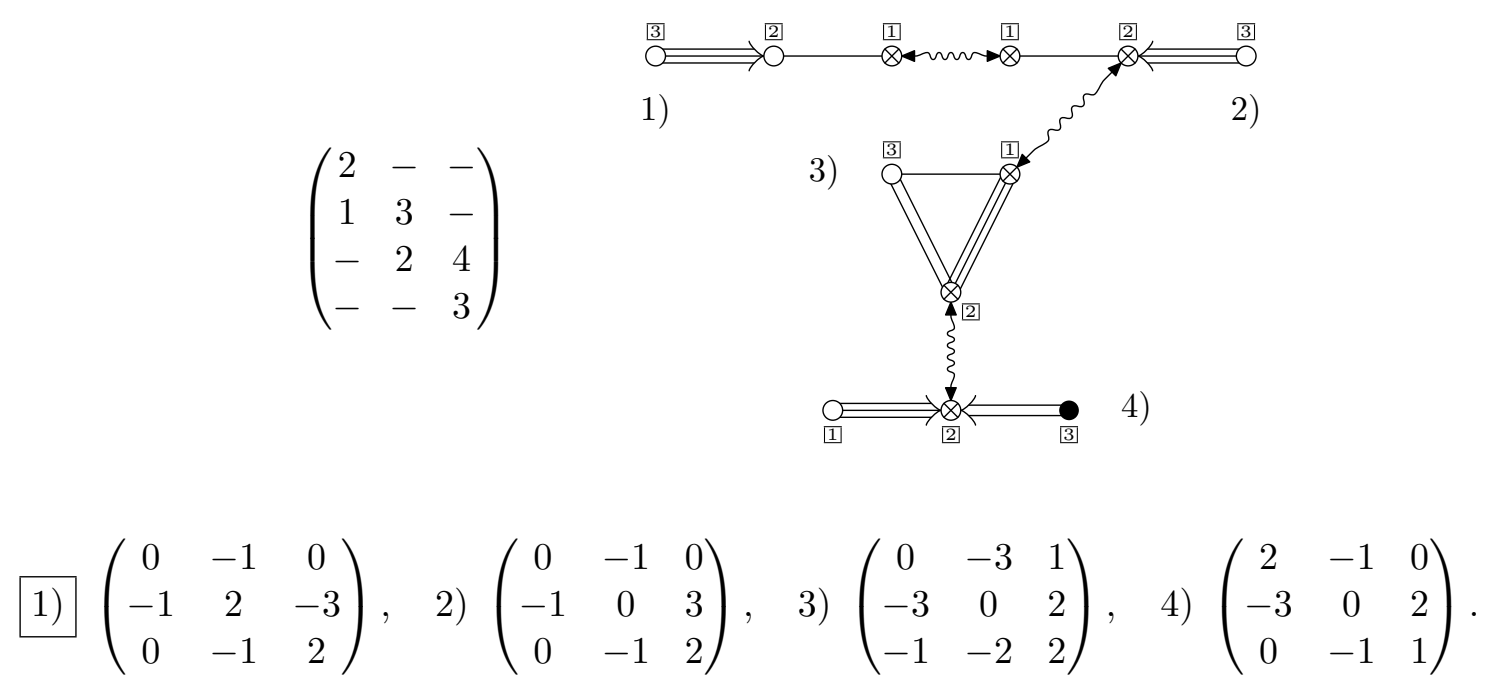

Recall that $\mathfrak{a} \mathfrak{b}(3)$ of sdim $=24 \mid 16$ has the following Cartan matrices

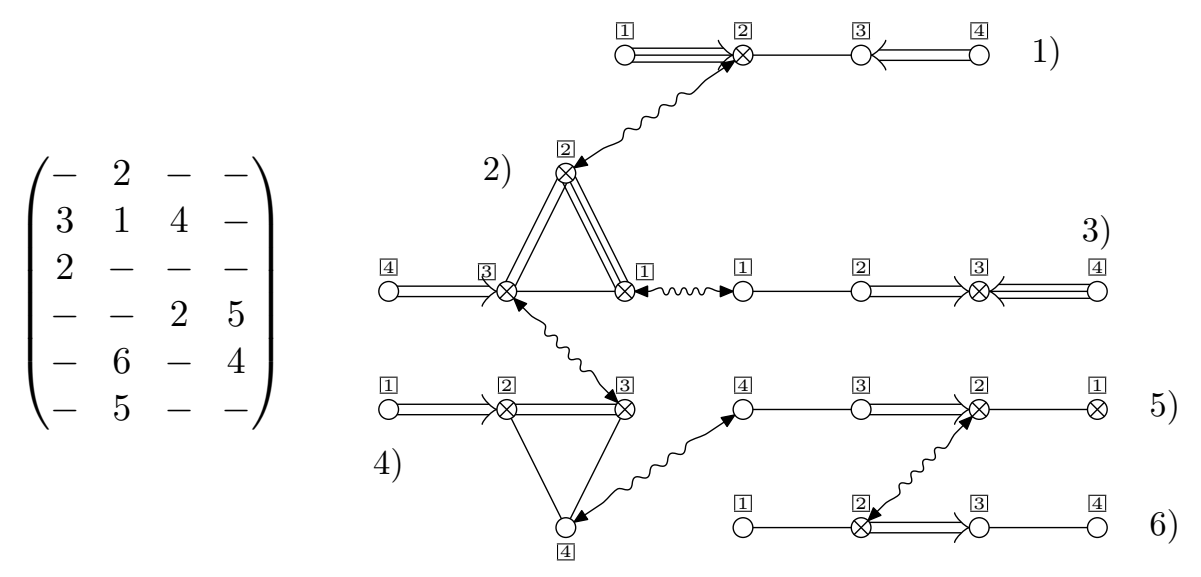
1) $\left(\begin{array}{cccc}2 & -1 & 0 & 0 \\ -3 & 0 & 1 & 0 \\ 0 & -1 & 2 & -2 \\ 0 & 0 & -1 & 2\end{array}\right)$
2) $\left(\begin{array}{cccc}0 & -3 & 1 & 0 \\ -3 & 0 & 2 & 0 \\ 1 & 2 & 0 & -2 \\ 0 & 0 & -1 & 2\end{array}\right)$
3) $\left(\begin{array}{cccc}2 & -1 & 0 & 0 \\ -1 & 2 & -1 & 0 \\ 0 & -2 & 0 & 3 \\ 0 & 0 & -1 & 2\end{array}\right)$,
4) $\left(\begin{array}{cccc}2 & -1 & 0 & 0 \\ -2 & 0 & 2 & -1 \\ 0 & 2 & 0 & -1 \\ 0 & -1 & -1 & 2\end{array}\right)$,
5) $\left(\begin{array}{cccc}0 & 1 & 0 & 0 \\ -1 & 0 & 2 & 0 \\ 0 & -1 & 2 & -1 \\ 0 & 0 & -1 & 2\end{array}\right)$
6) $\left(\begin{array}{cccc}2 & -1 & 0 & 0 \\ -1 & 2 & -1 & 0 \\ 0 & -2 & 2 & -1 \\ 0 & 0 & -1 & 0\end{array}\right)$.

Modular Lie algebras and Lie superalgebras.

$\underline{p=2 \text {, Lie algebras. Weisfeiler and Kac [54] }}$ discovered two new parametric families that we denote $\mathfrak{w} \mathfrak{k}(3 ; a)$ and $\mathfrak{w} \mathfrak{k}(4 ; a)$ (Weisfeiler and Kac algebras).

$\mathfrak{w} \mathfrak{k}(3 ; a)$, where $a \neq 0,-1$, of $\operatorname{dim} 18$ is a non-super version of $\mathfrak{o s p}(4 \mid 2 ; a)$ (although no osp exists for $p=2$ ); the dimension of its simple subquotient $\mathfrak{w k}(3 ; a)^{(1)} / \mathfrak{c}$ is equal to 16 ; the inequivalent Cartan matrices are:
1) $\left(\begin{array}{lll}\overline{0} & a & 0 \\ a & \overline{0} & 1 \\ 0 & 1 & \overline{0}\end{array}\right)$,
2) $\left(\begin{array}{ccc}\overline{0} & 1+a & a \\ 1+a & \overline{0} & 1 \\ a & 1 & \overline{0}\end{array}\right)$. 
$\mathfrak{w k}(4 ; a)$, where $a \neq 0,-1$, of $\operatorname{dim}=34$; the inequivalent Cartan matrices are:
1) $\left(\begin{array}{llll}\overline{0} & a & 0 & 0 \\ a & \overline{0} & 1 & 0 \\ 0 & 1 & \overline{0} & 1 \\ 0 & 0 & 1 & \overline{0}\end{array}\right)$,
2) $\left(\begin{array}{cccc}\overline{0} & 1 & 1+a & 0 \\ 1 & \overline{0} & a & 0 \\ a+1 & a & \overline{0} & a \\ 0 & 0 & a & \overline{0}\end{array}\right)$,
3) $\left(\begin{array}{cccc}\overline{0} & a & 0 & 0 \\ a & \overline{0} & a+1 & 0 \\ 0 & a+1 & \overline{0} & 1 \\ 0 & 0 & 1 & \overline{0}\end{array}\right)$.

Weisfeiler and Kac investigated also which of these algebras are isomorphic and the answer is as follows:

$$
\begin{aligned}
& \mathfrak{w k}(3 ; a) \simeq \mathfrak{w k}\left(3 ; a^{\prime}\right) \Longleftrightarrow a^{\prime}=\frac{\alpha a+\beta}{\gamma a+\delta}, \quad \text { where }\left(\begin{array}{ll}
\alpha & \beta \\
\gamma & \delta
\end{array}\right) \in \mathrm{SL}(2 ; \mathbb{Z} / 2), \\
& \mathfrak{w} \mathfrak{k}(4 ; a) \simeq \mathfrak{w k}\left(4 ; a^{\prime}\right) \Longleftrightarrow a^{\prime}=\frac{1}{a} .
\end{aligned}
$$

\subsubsection{2-structures on $\mathfrak{w k}$ algebras}

1) Observe that the center $\mathfrak{c}$ of $\mathfrak{w k}(3 ; a)$ is spanned by $a h_{1}+h_{3}$. The 2 -structure on $\mathfrak{w} \mathfrak{k}(3 ; a)$ is given by the conditions $\left(e_{\alpha}^{ \pm}\right)^{[2]}=0$ for all root vectors and the following ones:

a) For the matrix $B=(0,0,1)$ in (4.7) for the grading operator $d$, set:

$$
\begin{aligned}
& \left(\operatorname{ad}_{h_{1}}\right)^{[2]}=(1+a t) h_{1}+t h_{3} \equiv h_{1} \quad(\bmod \mathfrak{c}), \\
& \left(\operatorname{ad}_{h_{2}}\right)^{[2]}=a t h_{1}+h_{2}+t h_{3}+a(1+a) d \equiv h_{2}+a(1+a) d \quad(\bmod \mathfrak{c}), \\
& \left(\operatorname{ad}_{h_{3}}\right)^{[2]}=\left(a t+a^{2}\right) h_{1}+t h_{3} \equiv a^{2} h_{1} \quad(\bmod \mathfrak{c}), \\
& \left(\operatorname{ad}_{d}\right)^{[2]}=a t h_{1}+t h_{3}+d \equiv d \quad(\bmod \mathfrak{c})
\end{aligned}
$$

where $t$ is a parameter.

b) Taking $B=(1,0,0)$ in $(4.7)$ we get a more symmetric answer:

$$
\begin{aligned}
& \left(\operatorname{ad}_{h_{1}}\right)^{[2]}=(1+a t) h_{1}+t h_{3} \equiv h_{1} \quad(\bmod \mathfrak{c}), \\
& \left(\operatorname{ad}_{h_{2}}\right)^{[2]}=a t h_{1}+a h_{2}+t h_{3}+(1+a) d \equiv a h_{2}+(1+a) d \quad(\bmod \mathfrak{c}), \\
& \left(\operatorname{ad}_{h_{3}}\right)^{[2]}=\left(a t+a^{2}\right) h_{1}+t h_{3} \equiv a^{2} h_{1} \quad(\bmod \mathfrak{c}), \\
& \left(\operatorname{ad}_{d}\right)^{[2]}=a t h_{1}+t h_{3}+d \equiv d \quad(\bmod \mathfrak{c})
\end{aligned}
$$

(The expressions are somewhat different since we have chosen a different basis but on this simple Lie algebra the 2 -structure is unique.)

2) The 2 -structure on $\mathfrak{w k}(4 ; a)$ is given by the conditions $\left(e_{\alpha}^{ \pm}\right)^{[2]}=0$ for all root vectors and

$$
\begin{aligned}
& \left(\operatorname{ad}_{h_{1}}\right)^{[2]}=a h_{1}+(1+a) h_{4}, \\
& \left(\operatorname{ad}_{h_{2}}\right)^{[2]}=a h_{2}, \\
& \left(\operatorname{ad}_{h_{3}}\right)^{[2]}=h_{3}, \\
& \left(\operatorname{ad}_{h_{4}}\right)^{[2]}=h_{4} .
\end{aligned}
$$

$\underline{p=3, \text { Lie algebras. Brown }} 5$ algebras:

$$
\mathfrak{b r}(2, a) \text { with CM }\left(\begin{array}{cc}
2 & -1 \\
a & 2
\end{array}\right) \text { and } \mathfrak{b r}(2)=\lim _{-\frac{2}{a} \longrightarrow 0} " \mathfrak{b} \mathfrak{r}(2, a) \text { with } \mathrm{CM}\left(\begin{array}{cc}
2 & -1 \\
-1 & 0
\end{array}\right)
$$

The reflections change the value of the parameter, so

$$
\mathfrak{b r}(2, a) \simeq \mathfrak{b r}\left(2, a^{\prime}\right) \Longleftrightarrow a^{\prime}=-(1+a) .
$$

\footnotetext{
${ }^{5}$ To interpret the limit in $(9.9)$, set $\varepsilon=1+\frac{1}{\alpha}$, and $\mathfrak{b r}(2):=\mathfrak{b} \mathfrak{r}(2 ; \varepsilon)$ for $\varepsilon=1$.
} 


$$
1 \mathfrak{b r}(3) \text { with CM }\left(\begin{array}{ccc}
2 & -1 & 0 \\
-1 & 2 & -1 \\
0 & -1 & \overline{0}
\end{array}\right) \text { and } 2 \mathfrak{b} \mathfrak{r}(3) \text { with CM }\left(\begin{array}{ccc}
2 & -1 & 0 \\
-2 & 2 & -1 \\
0 & -1 & 0
\end{array}\right) \text {. }
$$

$\underline{p=3, \text { Lie superalgebras. }}$

Brown superalgebra $\mathfrak{b r j}(2 ; 3)$ of $\operatorname{sdim}=10 \mid 8$ (recently discovered in [17, Theorem 3.2(i)]; its Cartan matrices are first listed in [5]) has the following Cartan matrices
1) $\left(\begin{array}{cc}0 & -1 \\ -2 & 1\end{array}\right)$,
2) $\left(\begin{array}{cc}0 & -1 \\ -1 & \overline{0}\end{array}\right)$
3) $\left(\begin{array}{cc}1 & -1 \\ -1 & \overline{0}\end{array}\right)$

The Lie superalgebra $\mathfrak{b r j}(2 ; 3)$ is a super analog of the Brown algebra $\mathfrak{b r}(2)=\mathfrak{b r j}(2 ; 3)_{\overline{0}}$, its even part; $\mathfrak{b r j}(2 ; 3) \overline{1}=R\left(2 \pi_{1}\right)$ is irreducible $\mathfrak{b r j}(2 ; 3) \overline{0}^{-}$-module.

Elduque [17, 18, 13, 14] considered a particular case of the problem (9.1) and arranged the Lie (super)algebras he discovered in a Supermagic Square all its entries being of the form $\mathfrak{g}(A)$. These Elduque and Cunha superalgebras are, indeed, exceptional ones. For the complete list of their inequivalent Cartan matrices, reproduced here, see [3], where their presentation are also given; we also reproduce the description of the even and odd parts of these Lie superalgebras (all but one discovered by Elduque and whose description in terms of symmetric composition algebras is due to Elduque and Cunha), see Section 12.1.

$p=5$, Lie superalgebras. Brown superalgebra $\mathfrak{b r j}(2 ; 5)$ of $\operatorname{sdim}=10 \mid 12$, recently discovered in $\left[\overline{5]}\right.$, such that $\mathfrak{b r j}(2 ; 5)_{\overline{0}}=\mathfrak{s p}(4)$ and $\mathfrak{b r j}(2 ; 5)_{\overline{1}}=R\left(\pi_{1}+\pi_{2}\right)$ is an irreducible $\mathfrak{b r j}(2 ; 5) \overline{0}^{- \text {module }}{ }^{6}$. The Lie superalgebra $\mathfrak{b} \mathfrak{r j}(2 ; 5)$ has the following Cartan matrices:

$$
\left.\left(\begin{array}{ll}
2 & - \\
1 & -
\end{array}\right) \quad \text { 1) }\left(\begin{array}{cc}
0 & -1 \\
-2 & 1
\end{array}\right), \quad 2\right)\left(\begin{array}{cc}
0 & -1 \\
-3 & 2
\end{array}\right) .
$$

Elduque superalgebra $\mathfrak{e l}(5 ; 5)$ of $\operatorname{sdim}=55 \mid 32$, where $\mathfrak{e l}(5 ; 5)_{\overline{0}}=\mathfrak{o}(11)$ and $\mathfrak{e l}(5 ; 5)_{\overline{1}}=\operatorname{spin}_{11}$. Its inequivalent Cartan matrices, first described in [4], are as follows:

Instead of joining nodes by four segments in the cases where $A_{i j}=A_{j i}=1 \equiv-4 \bmod 5$ we use one dotted segment.

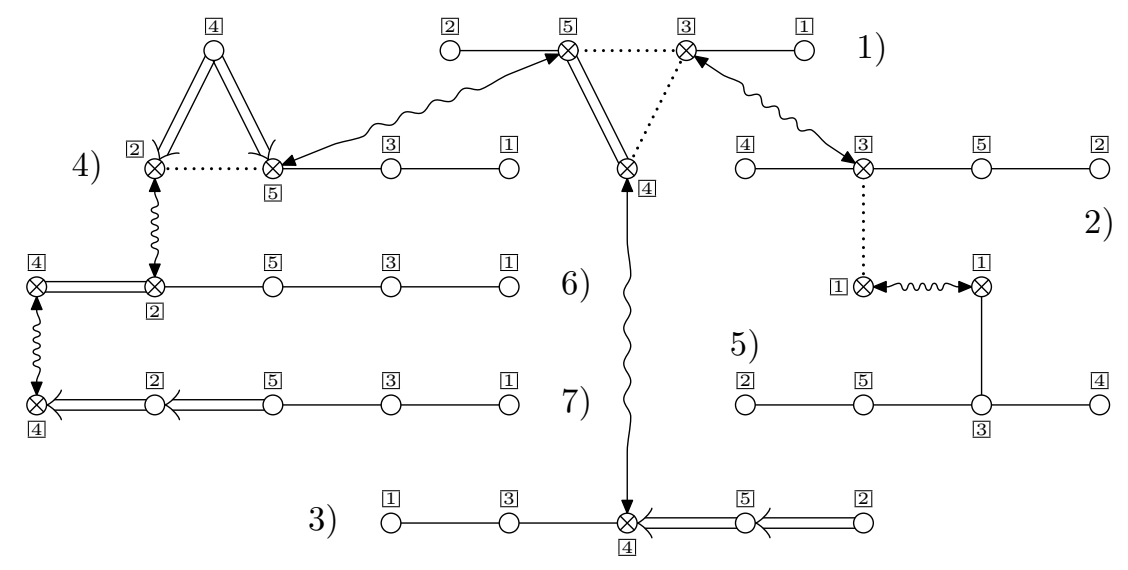

1) $\left.\left(\begin{array}{ccccc}2 & 0 & -1 & 0 & 0 \\ 0 & 2 & 0 & 0 & -1 \\ -1 & 0 & 0 & -4 & -4 \\ 0 & 0 & -4 & 0 & -2 \\ 0 & -1 & -4 & -2 & 0\end{array}\right), \quad 2\right)\left(\begin{array}{ccccc}0 & 0 & -4 & 0 & 0 \\ 0 & 2 & 0 & 0 & -1 \\ -4 & 0 & 0 & -1 & -1 \\ 0 & 0 & -1 & 2 & 0 \\ 0 & -1 & -1 & 0 & 2\end{array}\right)$,

\footnotetext{
${ }^{6}$ To the incredulous reader: The Cartan subalgebra of $\mathfrak{s p}(4)$ is generated by $h_{2}$ and $2 h_{1}+h_{2}$. The highest weight vector is $x_{10}=\left[\left[x_{2},\left[x_{2},\left[x_{1}, x_{2}\right]\right]\right],\left[\left[x_{1}, x_{2}\right],\left[x_{1}, x_{2}\right]\right]\right]$ and its weight is not a multiple of a fundamental weight, but $(1,1)$. We encounter several more instances of non-fundamental weights in descriptions of exceptions for $p=2$.
} 


$$
\begin{aligned}
& \left.3)\left(\begin{array}{ccccc}
2 & 0 & -1 & 0 & 0 \\
0 & 2 & 0 & 0 & -1 \\
-1 & 0 & 2 & -1 & 0 \\
0 & 0 & -1 & 0 & 2 \\
0 & -2 & 0 & -1 & 2
\end{array}\right), \quad 4\right)\left(\begin{array}{ccccc}
2 & 0 & -1 & 0 & 0 \\
0 & 0 & 0 & 2 & -4 \\
-1 & 0 & 2 & 0 & -1 \\
0 & -1 & 0 & 2 & -1 \\
0 & -4 & -1 & 2 & 0
\end{array}\right) \text {, } \\
& \left.5)\left(\begin{array}{ccccc}
0 & 0 & -1 & 0 & 0 \\
0 & 2 & 0 & 0 & -1 \\
-1 & 0 & 2 & -1 & -1 \\
0 & 0 & -1 & 2 & 0 \\
0 & -1 & -1 & 0 & 2
\end{array}\right), \quad 6\right)\left(\begin{array}{ccccc}
2 & 0 & -1 & 0 & 0 \\
0 & 0 & 0 & -2 & -1 \\
-1 & 0 & 2 & 0 & -1 \\
0 & -2 & 0 & 0 & 0 \\
0 & -1 & -1 & 0 & 2
\end{array}\right) \\
& \left.7)\left(\begin{array}{ccccc}
2 & 0 & -1 & 0 & 0 \\
0 & 2 & 0 & -1 & -2 \\
-1 & 0 & 2 & 0 & -1 \\
0 & 2 & 0 & 0 & 0 \\
0 & -1 & -1 & 0 & 2
\end{array}\right), \quad 8\right)\left(\begin{array}{ccccc}
- & - & 2 & 3 & 4 \\
5 & - & 1 & - & - \\
- & - & - & 1 & - \\
- & 6 & - & - & 1 \\
2 & - & - & - & - \\
- & 4 & - & 7 & - \\
- & - & - & 6 & -
\end{array}\right) .
\end{aligned}
$$

\subsection{Step 2: Studying $2 \times 2$ and $3 \times 3$ Cartan matrices}

1) We ask Mathematica to construct all possible matrices of a specific size. The matrices are not normalized and they must not be symmetrizable: we can not eliminate non-symmetrizable matrices at this stage. Fortunately, all $2 \times 2$ matrices are symmetrizable.

2) We ask Mathematica to eliminate the matrices with the following properties:

a) Matrices $A$ for whose submatrix $B$ we know that $\operatorname{dim} \mathfrak{g}(B)=\infty$;

b) decomposable matrices.

3) Matrices with a row in each that differ from each other by a nonzero factor are counted once, e.g.,

$$
\left(\begin{array}{ll}
1 & 1 \\
3 & 2
\end{array}\right) \cong\left(\begin{array}{ll}
2 & 2 \\
3 & 2
\end{array}\right) \cong\left(\begin{array}{ll}
6 & 6 \\
6 & 4
\end{array}\right)
$$

4) Equivalent matrices are counted once, where equivalence means that one matrix can be obtained from the other one by simultaneous transposition of rows and columns with the same numbers and the same parity. For example,

$$
\left(\begin{array}{llll}
0 & \alpha & 0 & 0 \\
\alpha & \overline{0} & 0 & 1 \\
0 & 0 & \overline{0} & 1 \\
0 & 1 & 1 & \overline{0}
\end{array}\right) \sim\left(\begin{array}{llll}
0 & \alpha & 1 & 0 \\
\alpha & \overline{0} & 0 & 0 \\
1 & 0 & \overline{0} & 1 \\
0 & 0 & 1 & \overline{0}
\end{array}\right) \sim\left(\begin{array}{cccc}
0 & 0 & 0 & 1 \\
0 & \overline{0} & \alpha & 1 \\
0 & \alpha & \overline{0} & 0 \\
1 & 1 & 0 & \overline{0}
\end{array}\right)
$$

At the substeps 1.1)-1.4) we thus get a store of Cartan matrices to be tested further.

$5)$ Now, we ask SuperLie, see [25], to construct the Lie superalgebras $\mathfrak{g}(A)$ up to certain dimension (say, 256). Having stored the Lie superalgebras $\mathfrak{g}(A)$ of dimension $<256$ we increase the range again if there are any algebras left (say, to 1024 or 2048). At this step, we conjecture that the dimension of any finite dimensional simple Lie (super)algebra of the form $\mathfrak{g}(A)$, where $A$ is of size $n \times n$, does not grow too rapidly with $n$. Say, at least, not as fast as $n^{10}$.

If the dimension of $\mathfrak{g}(A)$ increases accordingly, then we conjecture that $\mathfrak{g}(A)$ is infinite dimensional and this Lie superalgebra is put away for a while (but not completely eliminated as 
decomposable matrices that correspond to non-simple algebras: The progress of science might require soon to investigate how fast the dimension grows with $n$ : polynomially or faster).

6) For the stored Cartan matrices $A$, we have $\operatorname{dim} \mathfrak{g}(A)<\infty$. Once we get the full list all of such Cartan matrices of a given size, we have to check if $\mathfrak{g}(A)$ is simple, one by one.

7) The vectors of parities of the generators Pty $=\left(p_{1}, \ldots, p_{n}\right)$ are only considered of the form $(\overline{1}, \ldots, \overline{1}, \overline{0}, \ldots, \overline{0})$.

\subsubsection{The case of $2 \times 2$ Cartan matrices}

On the diagonal we may have $2, \overline{1}$ or $\overline{0}$, if the corresponding root is even; 0 or 1 if the root is odd. To be on the safe side, we redid the purely even case. We have the following options to consider:

$$
\begin{aligned}
& \operatorname{Pty}=(\overline{0}, \overline{0}) \quad \mathfrak{a}_{1} \quad\left(\begin{array}{cc}
2 & 2 a \\
2 b & 2
\end{array}\right) \simeq\left(\begin{array}{cc}
2 & 2 a \\
b & \overline{1}
\end{array}\right) \simeq\left(\begin{array}{cc}
\overline{1} & a \\
2 b & 2
\end{array}\right) \simeq\left(\begin{array}{cc}
\overline{1} & a \\
b & \overline{1}
\end{array}\right) \simeq\left(\begin{array}{cc}
b & a b \\
a b & a
\end{array}\right), \\
& \mathfrak{a}_{2} \quad\left(\begin{array}{cc}
\overline{1} & 2 a \\
-1 & \overline{0}
\end{array}\right), \quad \mathfrak{a}_{3} \quad\left(\begin{array}{cc}
\overline{0} & -1 \\
-1 & \overline{0}
\end{array}\right) \text {; } \\
& \operatorname{Pty}=(\overline{1}, \overline{0}) \quad \mathfrak{a}_{4} \quad\left(\begin{array}{cc}
0 & -1 \\
2 a & 2
\end{array}\right) \simeq\left(\begin{array}{cc}
0 & -1 \\
a & \overline{1}
\end{array}\right) \text {, } \\
& \mathfrak{a}_{5} \quad\left(\begin{array}{cc}
\overline{0} & -1 \\
-1 & 0
\end{array}\right), \quad \mathfrak{a}_{6} \quad\left(\begin{array}{cc}
1 & a \\
-1 & \overline{0}
\end{array}\right), \\
& \mathfrak{a}_{7} \quad\left(\begin{array}{cc}
1 & a \\
2 b & 2
\end{array}\right) \simeq\left(\begin{array}{cc}
1 & a \\
b & \frac{1}{1}
\end{array}\right) \\
& \operatorname{Pty}=(\overline{1}, \overline{1}) \quad \mathfrak{a}_{8} \quad\left(\begin{array}{cc}
1 & a \\
b & 1
\end{array}\right) \simeq\left(\begin{array}{cc}
b & a b \\
a b & a
\end{array}\right) \text {, } \\
& \mathfrak{a}_{9} \quad\left(\begin{array}{cc}
0 & -1 \\
-1 & 0
\end{array}\right), \quad \mathfrak{a}_{10} \quad\left(\begin{array}{cc}
0 & -1 \\
b & 1
\end{array}\right) \text {. }
\end{aligned}
$$

Obviously, some of these CMs had appeared in the study of (twisted) loops and the corresponding Kac-Moody Lie (super)algebras. One could expect that the reduction of the entries of $A$ modulo $p$ might yield a finite dimensional algebra, but this does not happen.

9.2.2. Lemma. If $A$ is non-symmetrizable, then $\operatorname{dim} \mathfrak{g}(A)=\infty$.

Proof. We prove this by inspection for $3 \times 3$ matrices, but the general case does not follow by reduction and induction: For example, for $p=2$ and the normalized non-symmetrizable matrix (here the value of $*$ is irrelevant)

$$
\left(\begin{array}{llll}
* & 1 & 1 & 0 \\
1 & * & 0 & 1 \\
1 & 0 & * & 1 \\
0 & 1 & a & *
\end{array}\right)
$$

where $a \neq 0,1$, or analogous $n \times n$ matrix whose Dynkin diagram is a loop, any $3 \times 3$ submatrix is symmetrizable.

To eliminate non-symmetrizable Cartan matrices, and any loops of length $>3$ in Dynkin diagrams, is, nevertheless, possible using Lemmas 3.1, 3.3, and 3.10, 3.11 of [54]. (Van de Leur [53] used these Lemmas for $p=0$.)

That was the idea of the proof. Now we pass to the case-by-case study. 


\subsection{Step 3: Studying $n \times n$ Cartan matrices for $n>3$}

By Lemma 9.2.2 we will assume that $A$ is symmetrizable. The idea is to use induction and the information found at each step.

9.3.1. Hypothesis. Each finite dimensional Lie superalgebra of the form $\mathfrak{g}(A)$ possesses a "simplest" Dynkin diagram - the one with only one odd node.

Therefore passing from $n \times n$ Cartan matrices to $(n+1) \times(n+1)$ Cartan matrices it suffices to consider just two types of $n \times n$ Cartan matrices: Purely even ones and the "simplest" ones with only one odd node on their Dynkin diagrams. To the latter ones only even node should be added.

\subsubsection{Further simplification of the algorithm}

Enlarging Cartan matrices by adding new row and column, we let, for $n>4$, its only non-zero elements occupy at most four slots (apart from the diagonal). Justification: Lemmas from $\S 3$ in [54] and Lemma 9.2.2.

Even this simplification still leaves lots of cases: To the 5 cases to be enlarged for Cartan matrices of size $\leq 8$ that we encounter for $p=0$, we have to add 16 super cases, each producing tens of possibilities in each of the major cases $p=2,3$ and 5 . To save several pages per each $n$ for each $p$, we have omitted the results of enlargements of each Cartan matrix and give only the final summary.

\subsection{On a quest for parametric families}

Even for $2 \times 2$ Cartan matrices we could have proceeded by "enlarging" but to be on the safe side we performed the selection independently. We considered only one or two parameters using the function called ParamSolve (of SuperLie, see [25]). It shows all cases where the division by an expression possibly equal to zero occurred. Every time SuperLie shows such a possibility we check it by hand; these possibility are algebraic equations of the form $\beta=f(\alpha)$, where $\alpha$ and $\beta$ are the parameters of the CM. We saw that whenever $\alpha$ and $\beta$ are generic $\operatorname{dim} \mathfrak{g}(A)$ grows too fast as compared with the height of the element (i.e., the number of brackets in expressions like $[a,[b,[c, d]]])$ that SuperLie should not exceed constructing a Lie (super)algebra. We did not investigate if the growth is polynomial or exponential, but definitely $\operatorname{dim} \mathfrak{g}(A)=\infty$. For each pair of singular values of parameters $\beta=f(\alpha)$, we repeat the computations again. In most cases, the algebra is infinite-dimensional, the exceptions being $\beta=\alpha+1$ that nicely correspond to some of CMs we already know, like $\mathfrak{w k}$ algebras.

For three parameters, we have equations of the form $\gamma=f(\alpha, \beta)$. For generic $\alpha$ and $\beta$. the Lie superalgebra $\mathfrak{g}(A)$ is infinite-dimensional. For the singular cases given by SuperLie, the constraints are of the form $\beta=g(\alpha)$. Now we face two possibilities: If $\gamma$ is a constant, then we just use the result of the previous step, when we dealt with two parameters. In the rare cases where $\gamma$ is not a constant and depends on the parameter $\alpha$, we have to recompute again and again the $\operatorname{dim} \mathfrak{g}(A)$ is infinite in these cases.

We find Cartan matrices of size $4 \times 4$ and larger by "enlarging". For $p=2$, we see that $3 \times 3$ $\mathrm{CMs}$ with parameters can be extended to $4 \times 4 \mathrm{CMs}$. However, $4 \times 4 \mathrm{CMs}$ cannot be extended to $5 \times 5 \mathrm{CMs}$ whose Lie (super)algebras are of finite dimension. For $p>2$, even $3 \times 3 \mathrm{CMs}$ cannot be extended.

\subsection{Super and modular cases: Summary of new features (as compared with simple Lie algebras over $\mathbb{C}$ )}

The super case, $p=0$. 
1) There are three types of nodes $(\bullet, \otimes$ and $\bigcirc)$,

2) there may occur a loop but only of length 3 ;

3) there is at most 1 parameter, but 1 parameter may occur;

4) to one algebra several inequivalent Cartan matrices can correspond.

The modular case. For Lie algebras, new features are the same as in the $p=0$ super case; additionally there appear new types of nodes $(\odot$ and $*)$.

\section{The answer: The case where $p>5$}

This case is the simplest one since it does not differ much from the $p=0$ case, where the answer is known.

Simple Lie algebras:

1) Lie algebras obtained from their $p=0$ analogs by reducing modulo $p$. We thus get

the CM versions of $\mathfrak{s l}$, namely: either simple $\mathfrak{s l}(n)$ or $\mathfrak{g l}(p n)$ whose "simple core" is $\mathfrak{p s l}(p n)$;

the orthogonal algebras $\mathfrak{o}(2 n+1)$ and $\mathfrak{o}(2 n)$;

the symplectic algebras $\mathfrak{s p}(2 n)$;

the exceptional algebras are $\mathfrak{g}(2), \mathfrak{f}(4), \mathfrak{e}(6), \mathfrak{e}(7), \mathfrak{e}(8)$.

Simple Lie superalgebras

Lie superalgebras obtained from their $p=0$ analogs by reducing modulo $p$. We thus get

1) the CM versions of $\mathfrak{s l}$, namely: either simple $\mathfrak{s l}(m \mid n)$ or $\mathfrak{g l}(a \mid p k+a)$ whose "simple core" is $\mathfrak{p s l}(a \mid p k+a)$ and $\mathfrak{p s l}^{(1)}(a \mid p k+a)$ if $a=k n$;

2) the ortho-symplectic algebras osp $(m \mid 2 n)$;

3) a parametric family $\mathfrak{o s p}(4 \mid 2 ; a)$;

4) the exceptional algebras are $\mathfrak{a} \mathfrak{g}(2)$ and $\mathfrak{a} \mathfrak{b}(3)$.

\section{The answer: The case where $p=5$}

Simple Lie algebras:

1) same as in Section 10 for $p=5$.

Simple Lie superalgebras

1) same as in Section 10 for $p=5$ and several new exceptions:

2) The Brown superalgebras $[5]: \mathfrak{b r j}(2 ; 5)$ such that $\mathfrak{b r j}(2 ; 5)_{\overline{0}}=\mathfrak{s p}(4)$ and the $\mathfrak{b r j}(2 ; 5)_{\overline{0}^{-}}$ module $\mathfrak{b r j}(2 ; 5)_{\overline{1}}=R\left(\pi_{1}+\pi_{2}\right)$ is irreducible with the highest weight vector

$$
x_{10}=\left[\left[x_{2},\left[x_{2},\left[x_{1}, x_{2}\right]\right]\right],\left[\left[x_{1}, x_{2}\right],\left[x_{1}, x_{2}\right]\right]\right]
$$

(for the CM 2): with the two Cartan matrices

$$
\left.\left(\begin{array}{ll}
2 & - \\
1 & -
\end{array}\right) \quad \text { 1) }\left(\begin{array}{cc}
0 & -1 \\
-2 & 1
\end{array}\right), \quad 2\right)\left(\begin{array}{cc}
0 & -1 \\
-3 & 2
\end{array}\right) \text {. }
$$

3) The Elduque superalgebra ell $(5 ; 5)$. Having found out one Cartan matrix of $\mathfrak{e l}(5 ; 5)$, we have listed them all, see 9.1.2. 


\section{The answer: The case where $p=3$}

Simple Lie algebras:

1) same as in Section 10 for $p=3$, except $\mathfrak{g}(2)$ which is not simple but contains a unique minimal ideal isomorphic to $\mathfrak{p s l}(3)$, and the following additional exceptions:

2) the Brown algebras $\mathfrak{b r}(2 ; a)$ and $\mathfrak{b r}(2)$ as well as $\mathfrak{b r}(3)$, see Section 9.1.

$\underline{\text { Simple Lie superalgebras }}$

1) same as in Section 10 for $p=3$ and $\mathfrak{e}(6)$ (with $\mathrm{CM}$ ) which is not simple but has a "simple core" $\mathfrak{e}(6) / \mathfrak{c}$;

2) the Brown superalgebras, see Section 9.1;

$3)$ the Elduque and Cunha superalgebras, see $[14,3]$. They are respective "enlargements" of the following Lie algebras (but can be also obtained by enlarging certain Lie superalgebras):

$\mathfrak{g}(2,3)(\mathfrak{g l}(3)$ yields $2 \mathfrak{g}(1,6)$ and $1 \mathfrak{g}(2,3))$ (with CM) has a simple core $\mathfrak{b} \mathfrak{j}:=\mathfrak{g}(2,3)^{(1)} / \mathfrak{c}$

$\mathfrak{g}(3,6)(\mathfrak{s l}(4)$ yields $7 \mathfrak{g}(3,6))$;

$\mathfrak{g}(3,3)(\mathfrak{s p}(6)$ yields $1 \mathfrak{g}(3,3)$ and $10 \mathfrak{g}(3,3))$;

$\mathfrak{g}(4,3)(\mathfrak{o}(7)$ yields $1 \mathfrak{g}(4,3))$

$\mathfrak{g}(8,3)(\mathfrak{f}(4)$ yields $1 \mathfrak{g}(8,3))$;

$\mathfrak{g}(2,6)(\mathfrak{s l}(5)$ yields $3 \mathfrak{g}(2,6))$ (with CM) has a simple core $\mathfrak{g}(2,6)^{(1)} / \mathfrak{c}$;

$\mathfrak{g}(4,6)(\mathfrak{g l}(6)$ yields $3 \mathfrak{g}(4,6)$ and $\mathfrak{o}(10)$ yields $7 \mathfrak{g}(4,6))$;

$\mathfrak{g}(6,6)(\mathfrak{o}(11)$ yields $21 \mathfrak{g}(6,6))$;

$\mathfrak{g}(8,6)(\mathfrak{s l}(7)$ yields $8 \mathfrak{g}(8,6)$ and $\mathfrak{e}(6)$ yields $3 \mathfrak{g}(8,6))$;

4) the Lie superalgebra $\mathfrak{e l}(5 ; 3)$ we have discovered is a $p=3$ version of the Elduque superalgebra el $(5 ; 5)$ : Their Cartan matrices (whose elements are represented by non-positive integers) 7) for $\mathfrak{e l}(5 ; 5)$ and 1) for $\mathfrak{e l}(5 ; 3)$ are identical after a permutation of indices (that is why we baptized $\mathfrak{e l}(5 ; 3)$ so). It can be obtained as an "enlargement" of any of the following Lie (super)algebras: $\mathfrak{s p}(8), \mathfrak{s l}(1 \mid 4), \mathfrak{s l}(2 \mid 3), \mathfrak{o s p}(4 \mid 4), \mathfrak{o s p}(6 \mid 2), \mathfrak{g}(3,3)$.

\subsection{Elduque and Cunha superalgebras: Systems of simple roots}

For details of description of Elduque and Cunha superalgebras in terms of symmetric composition algebras, see $[17,13,14]$. Here we consider the simple Elduque and Cunha superalgebras with Cartan matrix for $p=3$. In what follows, we list them using somewhat shorter notations as compared with the original ones: Hereafter $\mathfrak{g}(A, B)$ denotes the superalgebra occupying $(A, B)$ th slot in the Elduque Supermagic Square; the first Cartan matrix is usually the one given in [13], where only one Cartan matrix is given; the other matrices are obtained from the first one by means of reflections. Accordingly, $i \mathfrak{g}(A, B)$ is the shorthand for the realization of $\mathfrak{g}(A, B)$ by means of the $i$ th Cartan matrix. There are no instances of isotropic even reflections. On notation in the following tables, see Section 9.1.1.

\subsection{1 $\mathfrak{g}(1,6)$ of $\operatorname{sdim}=21 \mid 14$}

We have $\mathfrak{g}(1,6)_{\overline{0}}=\mathfrak{s p}(6)$ and $\mathfrak{g}(1,6)_{\overline{1}}=R\left(\pi_{3}\right)$.

$$
\left(\begin{array}{lll}
- & - & 2 \\
- & - & 1
\end{array}\right)
$$

1)

2)

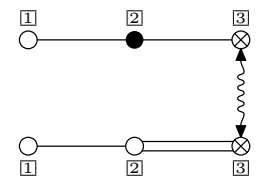


1) $\left.\left(\begin{array}{ccc}2 & -1 & 0 \\ -1 & 1 & -1 \\ 0 & -1 & 0\end{array}\right), \quad 2\right)\left(\begin{array}{ccc}2 & -1 & 0 \\ -1 & 2 & -2 \\ 0 & -2 & 0\end{array}\right)$.

12.1.2 $\mathfrak{g}(2,3)$ of $\operatorname{sdim}=12 / 10 \mid 14$

We have $\mathfrak{g}(2,3)_{\overline{0}}=\mathfrak{g l}(3) \oplus \mathfrak{s l}(2)$ and $\mathfrak{g}(2,3)_{\overline{1}}=\mathfrak{p s l}(3) \otimes \mathrm{id}$.

$$
\left(\begin{array}{ccc}
- & - & 2 \\
3 & 4 & 1 \\
2 & 5 & - \\
5 & 2 & - \\
4 & 3 & -
\end{array}\right)
$$

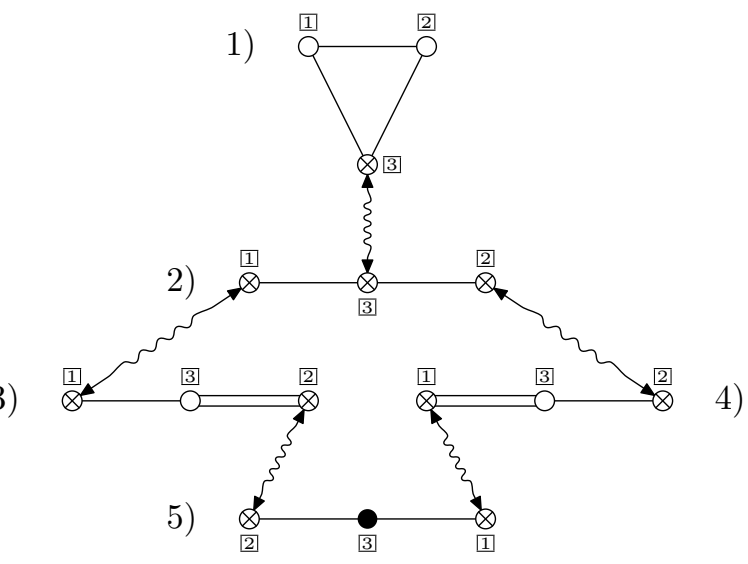

$$
\begin{aligned}
& \text { 1) } \left.\left(\begin{array}{ccc}
2 & -1 & -1 \\
-1 & 2 & -1 \\
-1 & -1 & 0
\end{array}\right), \quad \underline{2}\left(\begin{array}{ccc}
0 & 0 & -1 \\
0 & 0 & -1 \\
-1 & -1 & 0
\end{array}\right), \quad 3\right)\left(\begin{array}{ccc}
0 & 0 & -1 \\
0 & 0 & -2 \\
-1 & -2 & 2
\end{array}\right) \text {, } \\
& \text { 4) }\left(\begin{array}{ccc}
0 & 0 & -2 \\
0 & 0 & -1 \\
-2 & -1 & 2
\end{array}\right), \quad \underline{5}\left(\begin{array}{ccc}
0 & 0 & -1 \\
0 & 0 & -1 \\
-1 & -1 & 1
\end{array}\right) \text {. }
\end{aligned}
$$

\subsection{3 $\mathfrak{g}(3,6)$ of $\operatorname{sdim}=36 \mid 40$}

We have $\mathfrak{g}(3,6)_{\overline{0}}=\mathfrak{s p}(8)$ and $\mathfrak{g}(3,6)_{\overline{1}}=R\left(\pi_{3}\right)$.

$$
\begin{aligned}
& \left(\begin{array}{cccc}
2 & - & - & 3 \\
1 & 4 & - & 5 \\
5 & - & - & 1 \\
- & 2 & - & 6 \\
3 & 6 & - & 2 \\
- & 5 & 7 & 4 \\
- & - & 6 & -
\end{array}\right) \\
& \text { 1) }\left(\begin{array}{cccc}
0 & -1 & 0 & 0 \\
-1 & 2 & -1 & 0 \\
0 & -1 & 1 & -1 \\
0 & 0 & -1 & 0
\end{array}\right), \quad \underline{2}\left(\begin{array}{cccc}
0 & -1 & 0 & 0 \\
-1 & 0 & -1 & 0 \\
0 & -1 & 1 & -1 \\
0 & 0 & -1 & 0
\end{array}\right) \text {, } \\
& \text { 3) }\left(\begin{array}{cccc}
0 & -1 & 0 & 0 \\
-1 & 2 & -1 & 0 \\
0 & -1 & 2 & -2 \\
0 & 0 & -1 & 0
\end{array}\right) \\
& \left(\begin{array}{cccc}
2 & -1 & 0 & 0 \\
-1 & 0 & -2 & 0 \\
0 & -2 & 2 & -1 \\
0 & 0 & -1 & 0
\end{array}\right) \\
& \text { 5) }\left(\begin{array}{cccc}
0 & -1 & 0 & 0 \\
-2 & 0 & -1 & 0 \\
0 & -1 & 2 & -2 \\
0 & 0 & -1 & 0
\end{array}\right) \\
& \text { 6) } \left.\left(\begin{array}{cccc}
2 & -1 & 0 & 0 \\
-1 & 0 & -2 & 0 \\
0 & -2 & 0 & -2 \\
0 & 0 & -1 & 0
\end{array}\right), \quad 7\right)\left(\begin{array}{cccc}
2 & -1 & 0 & 0 \\
-1 & 2 & -1 & -1 \\
0 & -1 & 0 & -1 \\
0 & -1 & -1 & 2
\end{array}\right) \text {. }
\end{aligned}
$$


2)

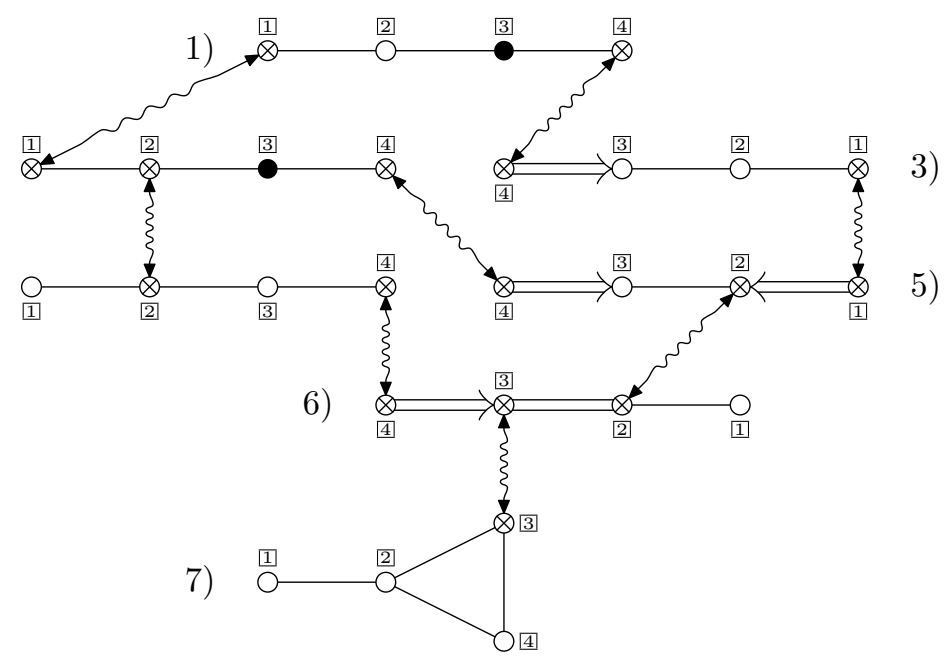

\subsection{4 $\mathfrak{g}(3,3)$ of $\operatorname{sdim}=23 / 21 \mid 16$}

We have $\mathfrak{g}(3,3)_{\overline{0}}=(\mathfrak{o}(7) \oplus \mathbb{K} z) \oplus \mathbb{K} d$ and $\mathfrak{g}(3,3)_{\overline{1}}=\left(\operatorname{spin}_{7}\right)_{+} \oplus\left(\operatorname{spin}_{7}\right)_{-} ;$the action of $d$ separates the summands - identical $\mathfrak{o}(7)$-modules $\operatorname{spin}_{7}$ - acting on one as the scalar multiplication by 1 , on the other one by -1 .

1)

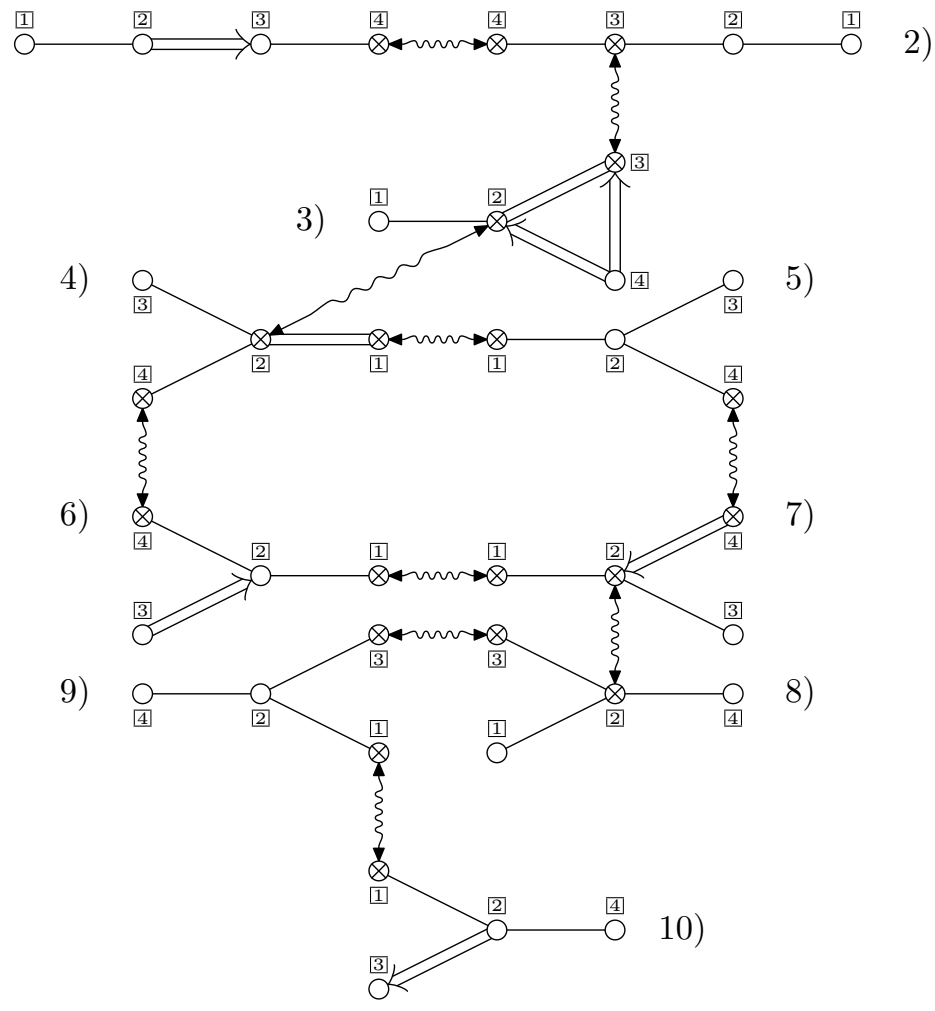




$$
\begin{aligned}
& \left.\left.\left(\begin{array}{cccc}
- & - & - & 2 \\
- & - & 3 & 1 \\
- & 4 & 2 & - \\
5 & 3 & - & 6 \\
4 & - & - & 7 \\
7 & - & - & 4
\end{array}\right), \quad 1\right)\left(\begin{array}{cccc}
2 & -1 & 0 & 0 \\
-1 & 2 & -1 & 0 \\
0 & -2 & 2 & -1 \\
0 & 0 & -1 & 0
\end{array}\right), \quad 2\right)\left(\begin{array}{cccc}
2 & -1 & 0 & 0 \\
-1 & 2 & -1 & 0 \\
0 & -1 & 0 & -1 \\
0 & 0 & -1 & 0
\end{array}\right), \\
& \left.\left.\left(\begin{array}{cccc}
7 & - & - & 4 \\
6 & 8 & - & 5 \\
- & 7 & 9 & - \\
10 & - & 8 & - \\
9 & - & - & -
\end{array}\right), \quad 3\right)\left(\begin{array}{cccc}
2 & -1 & 0 & 0 \\
-1 & 0 & -2 & -2 \\
0 & -2 & 0 & -2 \\
0 & -1 & -1 & 2
\end{array}\right), \quad 4\right)\left(\begin{array}{cccc}
0 & -1 & 0 & 0 \\
-2 & 0 & -1 & -1 \\
0 & -1 & 2 & 0 \\
0 & -1 & 0 & 0
\end{array}\right) \text {, } \\
& \text { 5) } \left.\left.\left(\begin{array}{cccc}
0 & -1 & 0 & 0 \\
-1 & 2 & -1 & -1 \\
0 & -1 & 2 & 0 \\
0 & -1 & 0 & 0
\end{array}\right), \quad 6\right)\left(\begin{array}{cccc}
0 & -1 & 0 & 0 \\
-1 & 2 & -2 & -1 \\
0 & -1 & 2 & 0 \\
0 & -1 & 0 & 0
\end{array}\right), \quad 7\right)\left(\begin{array}{cccc}
0 & -1 & 0 & 0 \\
-1 & 0 & -1 & -2 \\
0 & -1 & 2 & 0 \\
0 & -1 & 0 & 0
\end{array}\right) \text {, } \\
& \text { 8) } \left.\left.\left(\begin{array}{cccc}
2 & -1 & -1 & 0 \\
-2 & 0 & -2 & -1 \\
-1 & -1 & 0 & 0 \\
0 & -1 & 0 & 2
\end{array}\right), \quad 9\right)\left(\begin{array}{cccc}
0 & 0 & -1 & 0 \\
0 & 2 & -1 & -1 \\
-1 & -1 & 0 & 0 \\
0 & -1 & 0 & 2
\end{array}\right), \quad 10\right)\left(\begin{array}{cccc}
0 & 0 & -1 & 0 \\
0 & 2 & -1 & -1 \\
-1 & -2 & 2 & 0 \\
0 & -1 & 0 & 2
\end{array}\right) \text {. }
\end{aligned}
$$

\subsection{5 $\mathfrak{g}(4,3)$ of $\operatorname{sdim}=24 \mid 26$}

We have $\mathfrak{g}(4,3)_{\overline{0}}=\mathfrak{s p}(6) \oplus \mathfrak{s l}(2)$ and $\mathfrak{g}(4,3)_{\overline{1}}=R\left(\pi_{2}\right) \otimes \mathrm{id}$.

6)

1)

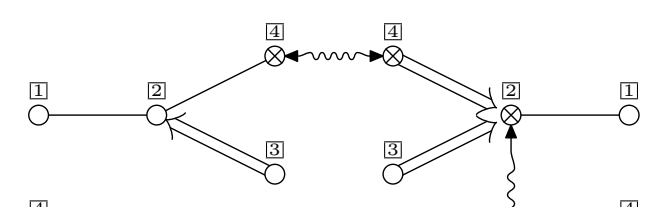

2)

8)

4)

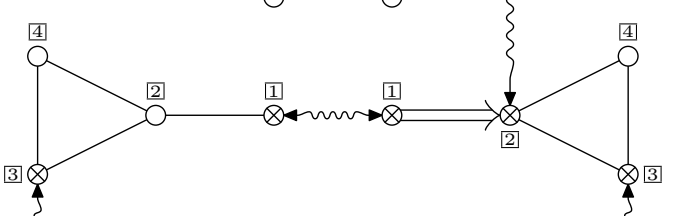

3)

9)

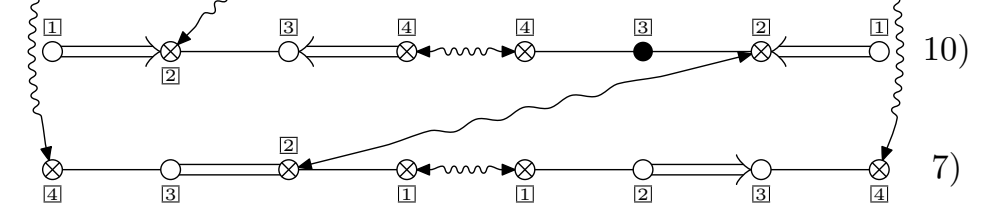

$$
\left(\begin{array}{cccc}
- & - & - & 2 \\
- & 3 & - & 1 \\
4 & 2 & 5 & - \\
3 & - & 6 & - \\
6 & - & 3 & 7 \\
5 & 8 & 4 & 9 \\
9 & - & - & 5 \\
- & 6 & - & 10 \\
7 & 10 & - & 6 \\
- & 9 & - & 8
\end{array}\right),
$$

1) $\left(\begin{array}{cccc}2 & -1 & 0 & 0 \\ -1 & 2 & -2 & -1 \\ 0 & -1 & 2 & 0 \\ 0 & -1 & 0 & 0\end{array}\right)$,

2) $\left(\begin{array}{cccc}2 & -1 & 0 & 0 \\ -1 & 0 & -2 & -2 \\ 0 & -1 & 2 & 0 \\ 0 & -1 & 0 & 0\end{array}\right)$,

3) $\left(\begin{array}{cccc}0 & -1 & 0 & 0 \\ -2 & 0 & -1 & -1 \\ 0 & -1 & 0 & -1 \\ 0 & -1 & -1 & 2\end{array}\right)$,

4) $\left(\begin{array}{cccc}0 & -1 & 0 & 0 \\ -1 & 2 & -1 & -1 \\ 0 & -1 & 0 & -1 \\ 0 & -1 & -1 & 2\end{array}\right)$, 

5) $\left(\begin{array}{cccc}0 & -1 & 0 & 0 \\ -1 & 2 & -1 & 0 \\ 0 & -1 & 0 & -1 \\ 0 & 0 & -1 & 0\end{array}\right), \quad \underline{6}\left(\begin{array}{cccc}0 & -1 & 0 & 0 \\ -1 & 0 & -2 & 0 \\ 0 & -1 & 0 & -1 \\ 0 & 0 & -1 & 0\end{array}\right)$,
7) $\left(\begin{array}{cccc}0 & -1 & 0 & 0 \\ -1 & 2 & -1 & 0 \\ 0 & -2 & 2 & -1 \\ 0 & 0 & -1 & 0\end{array}\right)$
8) $\left(\begin{array}{cccc}2 & -1 & 0 & 0 \\ -2 & 0 & -1 & 0 \\ 0 & -1 & 2 & -2 \\ 0 & 0 & -1 & 0\end{array}\right)$,
9) $\left(\begin{array}{cccc}0 & -1 & 0 & 0 \\ -1 & 0 & -2 & 0 \\ 0 & -2 & 2 & -1 \\ 0 & 0 & -1 & 0\end{array}\right)$,
10) $\left(\begin{array}{cccc}2 & -1 & 0 & 0 \\ -2 & 0 & -1 & 0 \\ 0 & -1 & 1 & -1 \\ 0 & 0 & -1 & 0\end{array}\right)$

\subsection{6 $\mathfrak{g}(2,6)$ of $\operatorname{sdim}=36 / 34 \mid 20$}

We have $\mathfrak{g}(2,6)_{\overline{0}}=\mathfrak{g l}(6)$ and $\mathfrak{g}(2,6)_{\overline{1}}=R\left(\pi_{3}\right)$.

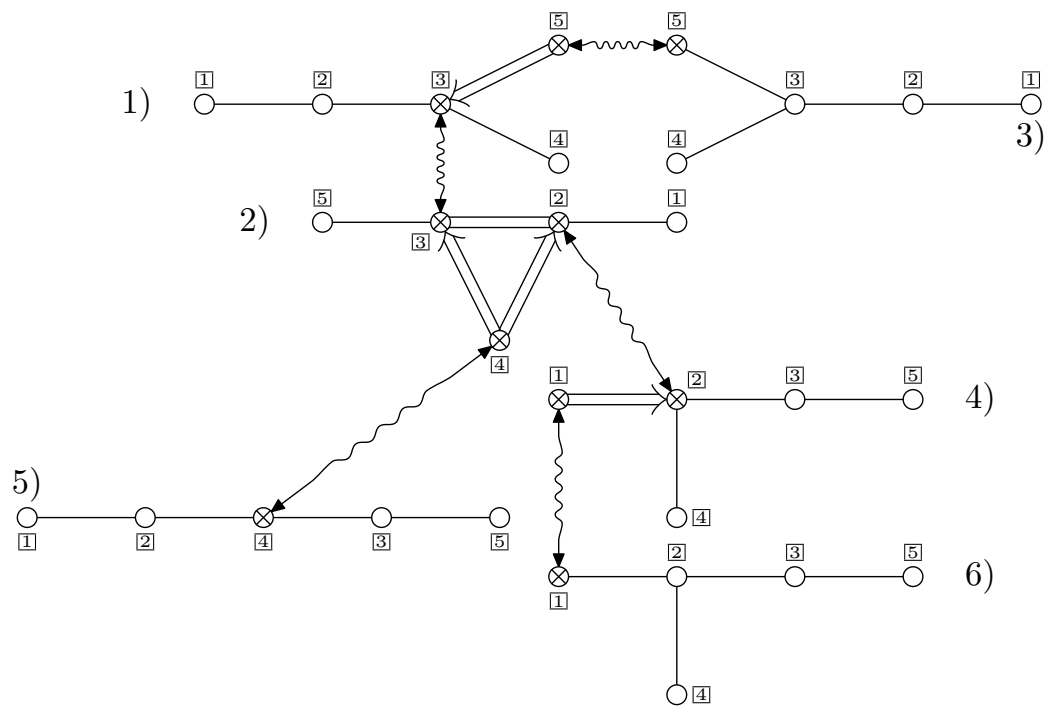

$$
\begin{aligned}
& \left.\left.\left(\begin{array}{ccccc}
- & - & 2 & - & 3 \\
- & 4 & 1 & 5 & - \\
- & - & - & - & 1 \\
6 & 2 & - & - & - \\
- & - & - & 2 & - \\
4 & - & - & - & -
\end{array}\right), \quad 1\right)\left(\begin{array}{ccccc}
2 & -1 & 0 & 0 & 0 \\
-1 & 2 & -1 & 0 & 0 \\
0 & -1 & 0 & -1 & -2 \\
0 & 0 & -1 & 2 & 0 \\
0 & 0 & -1 & 0 & 0
\end{array}\right), \quad 2\right)\left(\begin{array}{ccccc}
2 & -1 & 0 & 0 & 0 \\
-1 & 0 & -2 & -2 & 0 \\
0 & -2 & 0 & -2 & -1 \\
0 & -1 & -1 & 0 & 0 \\
0 & 0 & -1 & 0 & 2
\end{array}\right), \\
& \left.3)\left(\begin{array}{ccccc}
2 & -1 & 0 & 0 & 0 \\
-1 & 2 & -1 & 0 & 0 \\
0 & -1 & 2 & -1 & -1 \\
0 & 0 & -1 & 2 & 0 \\
0 & 0 & -1 & 0 & 0
\end{array}\right) \sim 6\right)\left(\begin{array}{ccccc}
0 & -1 & 0 & 0 & 0 \\
-1 & 2 & -1 & -1 & 0 \\
0 & -1 & 2 & 0 & -1 \\
0 & -1 & 0 & 2 & 0 \\
0 & 0 & -1 & 0 & 2
\end{array}\right), \\
& \text { 4) } \left.\left(\begin{array}{ccccc}
0 & -1 & 0 & 0 & 0 \\
-2 & 0 & -1 & -1 & 0 \\
0 & -1 & 2 & 0 & -1 \\
0 & -1 & 0 & 2 & 0 \\
0 & 0 & -1 & 0 & 2
\end{array}\right), \quad 5\right)\left(\begin{array}{ccccc}
2 & -1 & 0 & 0 & 0 \\
-1 & 2 & 0 & -1 & 0 \\
0 & 0 & 2 & -1 & -1 \\
0 & -1 & -1 & 0 & 0 \\
0 & 0 & -1 & 0 & 2
\end{array}\right) \text {. }
\end{aligned}
$$




\subsection{7 $\mathfrak{g}(8,3)$ of $\operatorname{sdim}=55 \mid 50$}

We have $\mathfrak{g}(8,3)_{\overline{0}}=\mathfrak{f}(4) \oplus \mathfrak{s l}(2)$ and $\mathfrak{g}(8,3)_{\overline{1}}=R\left(\pi_{4}\right) \otimes \mathfrak{i d}$.

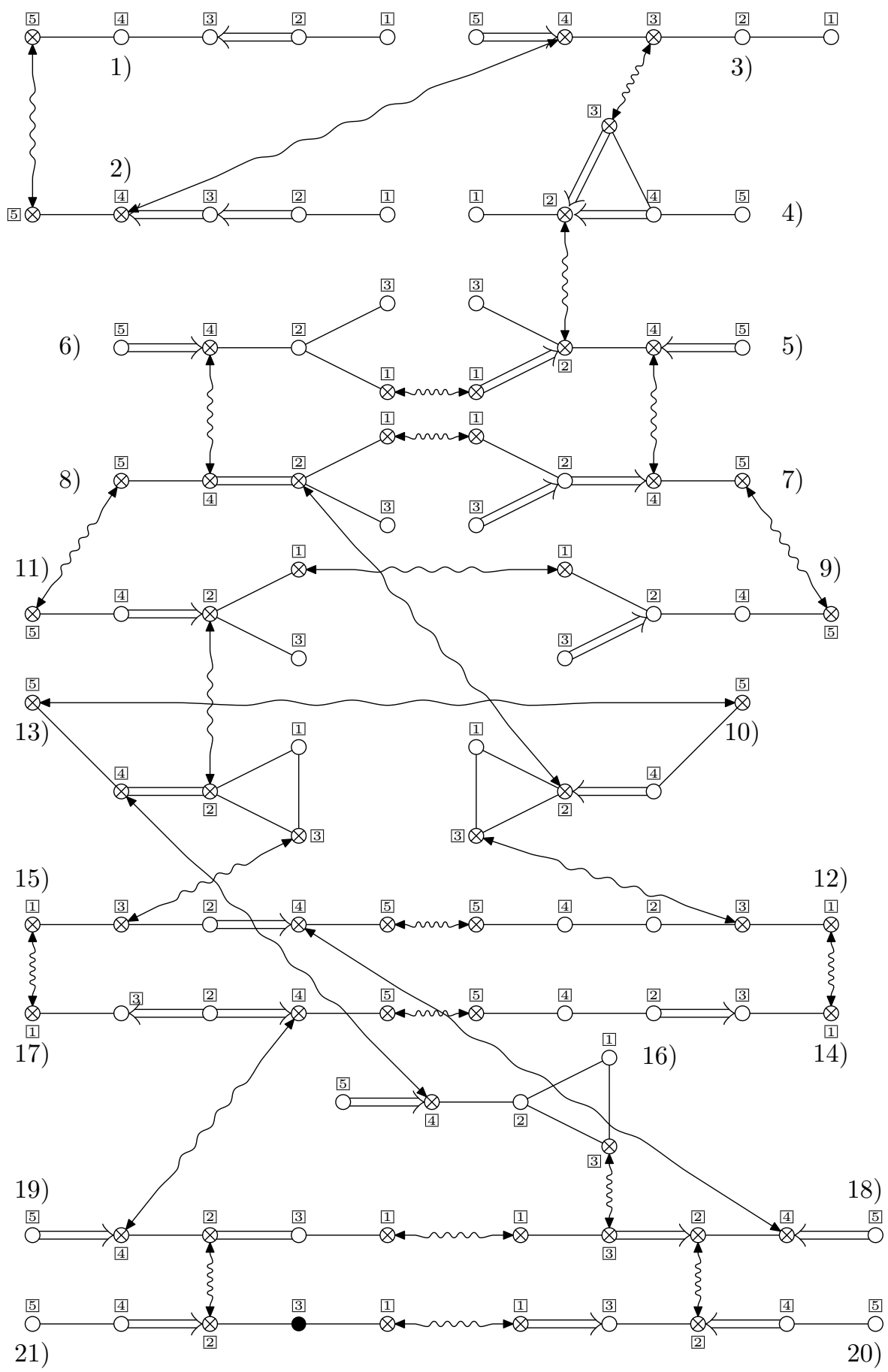




$$
\begin{aligned}
& \left(\begin{array}{ccccc}
- & - & - & - & 2 \\
- & - & - & 3 & 1 \\
- & - & 4 & 2 & - \\
- & 5 & 3 & - & - \\
6 & 4 & - & 7 & - \\
5 & - & - & 8 & - \\
8 & - & - & 5 & 9 \\
7 & 10 & - & 6 & 11 \\
11 & - & - & - & 7 \\
- & 8 & 12 & - & 13 \\
9 & 13 & - & - & 8 \\
14 & - & 10 & - & 15 \\
- & 11 & 15 & 16 & 10 \\
12 & - & - & - & 17 \\
17 & - & 13 & 18 & 12 \\
- & - & 18 & 13 & - \\
15 & - & - & 19 & 14 \\
19 & 20 & 16 & 15 & - \\
18 & 21 & - & 17 & - \\
21 & 18 & - & - & - \\
20 & 19 & - & - & -
\end{array}\right), \\
& \text { 1) }\left(\begin{array}{ccccc}
2 & -1 & 0 & 0 & 0 \\
-1 & 2 & -1 & 0 & 0 \\
0 & -2 & 2 & -1 & 0 \\
0 & 0 & -1 & 2 & -1 \\
0 & 0 & 0 & 1 & 0
\end{array}\right) \\
& \text { 5) } \\
& \left(\begin{array}{ccccc}
0 & -1 & 0 & 0 & 0 \\
-2 & 0 & -1 & -1 & 0 \\
0 & -1 & 2 & 0 & 0 \\
0 & -1 & 0 & 0 & -2 \\
0 & 0 & 0 & -1 & 2
\end{array}\right) \\
& \text { 2) }\left(\begin{array}{ccccc}
2 & -1 & 0 & 0 & 0 \\
-1 & 2 & -1 & 0 & 0 \\
0 & -2 & 2 & -1 & 0 \\
0 & 0 & -2 & 0 & -1 \\
0 & 0 & 0 & -1 & 0
\end{array}\right) \text {, 6) }\left(\begin{array}{ccccc}
0 & -1 & 0 & 0 & 0 \\
-1 & 2 & -1 & -1 & 0 \\
0 & -1 & 2 & 0 & 0 \\
0 & -1 & 0 & 0 & -2 \\
0 & 0 & 0 & -1 & 2
\end{array}\right) \text {, } \\
& 3)\left(\begin{array}{ccccc}
2 & -1 & 0 & 0 & 0 \\
-1 & 2 & -1 & 0 & 0 \\
0 & -1 & 0 & -1 & 0 \\
0 & 0 & -1 & 0 & -2 \\
0 & 0 & 0 & -1 & 2
\end{array}\right) \text {, 7) }\left(\begin{array}{ccccc}
0 & -1 & 0 & 0 & 0 \\
-1 & 2 & -2 & -1 & 0 \\
0 & -1 & 2 & 0 & 0 \\
0 & -2 & 0 & 0 & -1 \\
0 & 0 & 0 & -1 & 0
\end{array}\right) \text {, } \\
& \text { 4) }\left(\begin{array}{ccccc}
2 & -1 & 0 & 0 & 0 \\
-1 & 0 & -2 & -2 & 0 \\
0 & -1 & 0 & -1 & 0 \\
0 & -1 & -1 & 2 & -1 \\
0 & 0 & 0 & -1 & 2
\end{array}\right) \text {, 8) }\left(\begin{array}{ccccc}
0 & -1 & 0 & 0 & 0 \\
-1 & 0 & -1 & -2 & 0 \\
0 & -1 & 2 & 0 & 0 \\
0 & -2 & 0 & 0 & -1 \\
0 & 0 & 0 & -1 & 0
\end{array}\right) \text {, } \\
& \left.9)\left(\begin{array}{ccccc}
0 & -1 & 0 & 0 & 0 \\
-1 & 2 & -2 & -1 & 0 \\
0 & -1 & 2 & 0 & 0 \\
0 & -1 & 0 & 2 & -1 \\
0 & 0 & 0 & -1 & 0
\end{array}\right), \quad \text { 10) }\left(\begin{array}{ccccc}
2 & -1 & -1 & 0 & 0 \\
1 & 0 & 1 & 2 & 0 \\
1 & 1 & 0 & 0 & 0 \\
0 & -1 & 0 & 2 & -1 \\
0 & 0 & 0 & -1 & 0
\end{array}\right), \quad 11\right)\left(\begin{array}{ccccc}
0 & -1 & 0 & 0 & 0 \\
-1 & 0 & -1 & -2 & 0 \\
0 & -1 & 2 & 0 & 0 \\
0 & -1 & 0 & 2 & -1 \\
0 & 0 & 0 & -1 & 0
\end{array}\right) \text {, } \\
& \left.\left.12)\left(\begin{array}{ccccc}
0 & 0 & -1 & 0 & 0 \\
0 & 2 & -1 & -1 & 0 \\
-1 & -1 & 0 & 0 & 0 \\
0 & -1 & 0 & 2 & -1 \\
0 & 0 & 0 & -1 & 0
\end{array}\right), \quad 13\right)\left(\begin{array}{ccccc}
2 & -1 & -1 & 0 & 0 \\
-1 & 0 & -1 & -2 & 0 \\
-1 & -1 & 0 & 0 & 0 \\
0 & -2 & 0 & 0 & -1 \\
0 & 0 & 0 & -1 & 0
\end{array}\right), \quad 14\right)\left(\begin{array}{ccccc}
0 & 0 & -1 & 0 & 0 \\
0 & 2 & -1 & -1 & 0 \\
-1 & -2 & 2 & 0 & 0 \\
0 & -1 & 0 & 2 & -1 \\
0 & 0 & 0 & -1 & 0
\end{array}\right) \text {, } \\
& \text { 15) } \left.\left.\left(\begin{array}{ccccc}
0 & 0 & -1 & 0 & 0 \\
0 & 2 & -1 & -1 & 0 \\
-1 & -1 & 0 & 0 & 0 \\
0 & -2 & 0 & 0 & -1 \\
0 & 0 & 0 & -1 & 0
\end{array}\right), \quad 16\right)\left(\begin{array}{ccccc}
2 & -1 & -1 & 0 & 0 \\
-1 & 2 & -1 & -1 & 0 \\
-1 & -1 & 0 & 0 & 0 \\
0 & -1 & 0 & 0 & -2 \\
0 & 0 & 0 & -1 & 2
\end{array}\right), \quad 17\right)\left(\begin{array}{ccccc}
0 & 0 & -1 & 0 & 0 \\
0 & 2 & -1 & -1 & 0 \\
-1 & -2 & 2 & 0 & 0 \\
0 & -2 & 0 & 0 & -1 \\
0 & 0 & 0 & -1 & 0
\end{array}\right) \text {, } \\
& \left.\left.18)\left(\begin{array}{ccccc}
0 & 0 & -1 & 0 & 0 \\
0 & 0 & -2 & -1 & 0 \\
-1 & -1 & 0 & 0 & 0 \\
0 & -1 & 0 & 0 & -2 \\
0 & 0 & 0 & -1 & 2
\end{array}\right), \quad 19\right)\left(\begin{array}{ccccc}
0 & 0 & -1 & 0 & 0 \\
0 & 0 & -2 & -1 & 0 \\
-1 & -2 & 2 & 0 & 0 \\
0 & -1 & 0 & 0 & -2 \\
0 & 0 & 0 & -1 & 2
\end{array}\right), \quad 20\right)\left(\begin{array}{ccccc}
0 & 0 & -1 & 0 & 0 \\
0 & 0 & -1 & -2 & 0 \\
-2 & -1 & 2 & 0 & 0 \\
0 & -1 & 0 & 2 & -1 \\
0 & 0 & 0 & -1 & 2
\end{array}\right) \text {, } \\
& \text { 21) }\left(\begin{array}{ccccc}
0 & 0 & -1 & 0 & 0 \\
0 & 0 & -1 & -2 & 0 \\
-1 & -1 & 1 & 0 & 0 \\
0 & -1 & 0 & 2 & -1 \\
0 & 0 & 0 & -1 & 2
\end{array}\right) \text {. }
\end{aligned}
$$




\subsection{8 $\mathfrak{g}(4,6)$ of $\operatorname{sdim}=66 \mid 32$}

We have $\mathfrak{g}(4,6)_{\overline{0}}=\mathfrak{o}(12)$ and $\mathfrak{g}(4,6)_{\overline{1}}=R\left(\pi_{5}\right)$.

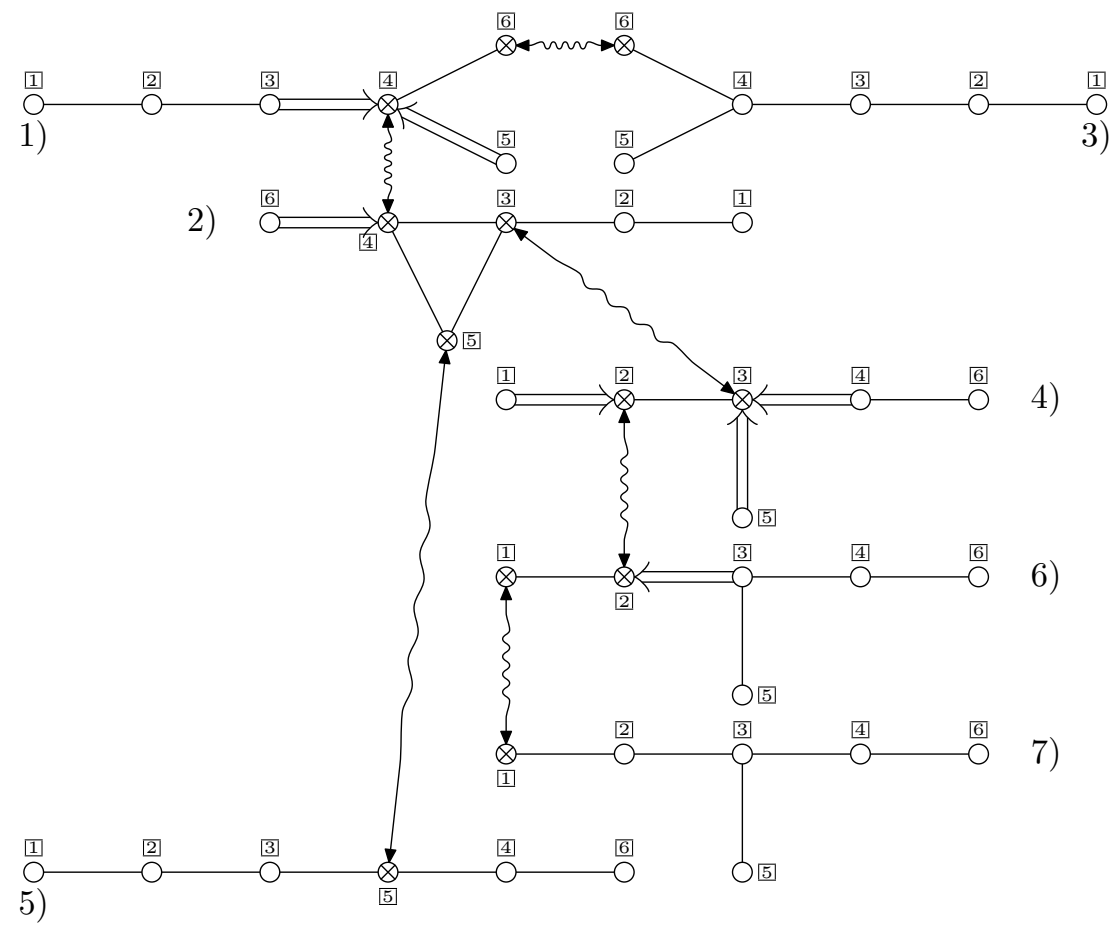

$$
\begin{aligned}
& \left.\left(\begin{array}{cccccc}
- & - & - & 2 & - & 3 \\
- & - & 4 & 1 & 5 & - \\
- & - & - & - & - & 1 \\
- & 6 & 2 & - & - & - \\
- & - & - & - & 2 & - \\
7 & 4 & - & - & - & - \\
6 & - & - & - & - & -
\end{array}\right), \quad 1\right)\left(\begin{array}{cccccc}
2 & -1 & 0 & 0 & 0 & 0 \\
-1 & 2 & -1 & 0 & 0 & 0 \\
0 & -1 & 2 & -1 & 0 & 0 \\
0 & 0 & -2 & 0 & -2 & -1 \\
0 & 0 & 0 & -1 & 2 & 0 \\
0 & 0 & 0 & -1 & 0 & 0
\end{array}\right), \\
& \left.2)\left(\begin{array}{cccccc}
2 & -1 & 0 & 0 & 0 & 0 \\
-1 & 2 & -1 & 0 & 0 & 0 \\
0 & -2 & 0 & -1 & -1 & 0 \\
0 & 0 & -1 & 0 & -1 & -2 \\
0 & 0 & -1 & -1 & 0 & 0 \\
0 & 0 & 0 & -1 & 0 & 2
\end{array}\right), \quad 3\right)\left(\begin{array}{cccccc}
2 & -1 & 0 & 0 & 0 & 0 \\
-1 & 2 & -1 & 0 & 0 & 0 \\
0 & -1 & 2 & -1 & 0 & 0 \\
0 & 0 & -1 & 2 & -1 & -1 \\
0 & 0 & 0 & -1 & 2 & 0 \\
0 & 0 & 0 & -1 & 0 & 0
\end{array}\right) \text {, } \\
& \left.4)\left(\begin{array}{cccccc}
2 & -1 & 0 & 0 & 0 & 0 \\
-2 & 0 & -1 & 0 & 0 & 0 \\
0 & -1 & 0 & -2 & -2 & 0 \\
0 & 0 & -1 & 2 & 0 & -1 \\
0 & 0 & -1 & 0 & 2 & 0 \\
0 & 0 & 0 & -1 & 0 & 2
\end{array}\right), 5\right)\left(\begin{array}{cccccc}
2 & -1 & 0 & 0 & 0 & 0 \\
-1 & 2 & -1 & 0 & 0 & 0 \\
0 & -1 & 2 & 0 & -1 & 0 \\
0 & 0 & 0 & 2 & -1 & -1 \\
0 & 0 & -1 & -1 & 0 & 0 \\
0 & 0 & 0 & -1 & 0 & 2
\end{array}\right) \text {, } \\
& \left.6)\left(\begin{array}{cccccc}
0 & -1 & 0 & 0 & 0 & 0 \\
-1 & 0 & -2 & 0 & 0 & 0 \\
0 & -1 & 2 & -1 & -1 & 0 \\
0 & 0 & -1 & 2 & 0 & -1 \\
0 & 0 & -1 & 0 & 2 & 0 \\
0 & 0 & 0 & -1 & 0 & 2
\end{array}\right), \quad 7\right)\left(\begin{array}{cccccc}
0 & -1 & 0 & 0 & 0 & 0 \\
-1 & 2 & -1 & 0 & 0 & 0 \\
0 & -1 & 2 & -1 & -1 & 0 \\
0 & 0 & -1 & 2 & 0 & -1 \\
0 & 0 & -1 & 0 & 2 & 0 \\
0 & 0 & 0 & -1 & 0 & 2
\end{array}\right) .
\end{aligned}
$$




\subsection{9 $\mathfrak{g}(6,6)$ of $\operatorname{sdim}=78 \mid 64$}

We have $\mathfrak{g}(6,6)_{\overline{0}}=\mathfrak{o}(13)$ and $\mathfrak{g}(6,6)_{\overline{1}}=\operatorname{spin}_{13}$.

$$
\begin{aligned}
& \left(\begin{array}{cccccc}
2 & 3 & - & 4 & - & 5 \\
1 & - & - & 6 & - & 7 \\
- & 1 & 8 & 9 & - & 10 \\
6 & 9 & 11 & 1 & 12 & - \\
7 & 10 & - & - & - & 1 \\
4 & - & 13 & 2 & 14 & - \\
5 & - & - & - & - & 2 \\
- & - & 3 & - & - & 15 \\
- & 4 & - & 3 & 16 & - \\
- & 5 & 15 & - & - & 3 \\
13 & - & 4 & - & - & - \\
14 & 16 & - & - & 4 & - \\
11 & 17 & 6 & - & - & - \\
12 & - & - & - & 6 & - \\
- & - & 10 & 18 & - & 8 \\
- & 12 & 19 & - & 9 & - \\
- & 13 & - & - & - & - \\
- & - & - & 15 & 20 & - \\
- & - & 16 & - & - & - \\
- & - & - & - & 18 & 21 \\
- & - & - & - & - & 20
\end{array}\right) \\
& \text { 1) }\left(\begin{array}{cccccc}
0 & -1 & 0 & 0 & 0 & 0 \\
-1 & 0 & -2 & 0 & 0 & 0 \\
0 & -1 & 2 & -1 & 0 & 0 \\
0 & 0 & -2 & 0 & -2 & -1 \\
0 & 0 & 0 & -1 & 2 & 0 \\
0 & 0 & 0 & -1 & 0 & 0
\end{array}\right) \\
& \text { 3) } \\
& \left(\begin{array}{cccccc}
2 & -1 & 0 & 0 & 0 & 0 \\
-2 & 0 & -1 & 0 & 0 & 0 \\
0 & -1 & 0 & -2 & 0 & 0 \\
0 & 0 & -2 & 0 & -2 & -1 \\
0 & 0 & 0 & -1 & 2 & 0 \\
0 & 0 & 0 & -1 & 0 & 0
\end{array}\right) \\
& \text { 4) } \\
& \left.\left(\begin{array}{cccccc}
0 & -1 & 0 & 0 & 0 & 0 \\
-1 & 0 & -2 & 0 & 0 & 0 \\
0 & -2 & 0 & -1 & -1 & 0 \\
0 & 0 & -1 & 0 & -1 & -2 \\
0 & 0 & -1 & -1 & 0 & 0 \\
0 & 0 & 0 & -1 & 0 & 2
\end{array}\right), \quad 5\right)\left(\begin{array}{cccccc}
0 & -1 & 0 & 0 & 0 & 0 \\
-1 & 0 & -2 & 0 & 0 & 0 \\
0 & -1 & 2 & -1 & 0 & 0 \\
0 & 0 & -1 & 2 & -1 & -1 \\
0 & 0 & 0 & -1 & 2 & 0 \\
0 & 0 & 0 & -1 & 0 & 0
\end{array}\right) \\
& \text { 6) } \\
& \left(\begin{array}{cccccc}
0 & -1 & 0 & 0 & 0 & 0 \\
-1 & 2 & -1 & 0 & 0 & 0 \\
0 & -2 & 0 & -1 & -1 & 0 \\
0 & 0 & -1 & 0 & -1 & -2 \\
0 & 0 & -1 & -1 & 0 & 0 \\
0 & 0 & 0 & -1 & 0 & 2
\end{array}\right) \\
& \left(\begin{array}{cccccc}
0 & -1 & 0 & 0 & 0 & 0 \\
-1 & 2 & -1 & 0 & 0 & 0 \\
0 & -1 & 2 & -1 & 0 & 0 \\
0 & 0 & -1 & 2 & -1 & -1 \\
0 & 0 & 0 & -1 & 2 & 0 \\
0 & 0 & 0 & -1 & 0 & 0
\end{array}\right) \\
& \text { 8) }\left(\begin{array}{cccccc}
2 & -1 & 0 & 0 & 0 & 0 \\
-1 & 2 & -1 & 0 & 0 & 0 \\
0 & -2 & 0 & -1 & 0 & 0 \\
0 & 0 & -1 & 2 & -2 & -1 \\
0 & 0 & 0 & -1 & 2 & 0 \\
0 & 0 & 0 & -1 & 0 & 0
\end{array}\right) \text {, } \\
& \left(\begin{array}{cccccc}
2 & -1 & 0 & 0 & 0 & 0 \\
-2 & 0 & -1 & 0 & 0 & 0 \\
0 & -1 & 2 & -1 & -1 & 0 \\
0 & 0 & -1 & 0 & -1 & -2 \\
0 & 0 & -1 & -1 & 0 & 0 \\
0 & 0 & 0 & -1 & 0 & 2
\end{array}\right) \\
& \text { 10) }\left(\begin{array}{cccccc}
2 & -1 & 0 & 0 & 0 & 0 \\
-2 & 0 & -1 & 0 & 0 & 0 \\
0 & -1 & 0 & -2 & 0 & 0 \\
0 & 0 & -1 & 2 & -1 & -1 \\
0 & 0 & 0 & -1 & 2 & 0 \\
0 & 0 & 0 & -1 & 0 & 0
\end{array}\right) \text {, } \\
& \text { 11) }\left(\begin{array}{cccccc}
0 & -1 & 0 & 0 & 0 & 0 \\
-1 & 2 & -1 & 0 & 0 & 0 \\
0 & -1 & 0 & -2 & -2 & 0 \\
0 & 0 & -1 & 2 & 0 & -1 \\
0 & 0 & -1 & 0 & 2 & 0 \\
0 & 0 & 0 & -1 & 0 & 2
\end{array}\right) \text {, } \\
& \text { 12) } \\
& \left(\begin{array}{cccccc}
0 & -1 & 0 & 0 & 0 & 0 \\
-1 & 0 & -2 & 0 & 0 & 0 \\
0 & -1 & 2 & 0 & -1 & 0 \\
0 & 0 & 0 & 2 & -1 & -1 \\
0 & 0 & -1 & -1 & 0 & 0 \\
0 & 0 & 0 & -1 & 0 & 2
\end{array}\right) \\
& \text { 13) }\left(\begin{array}{cccccc}
0 & -1 & 0 & 0 & 0 & 0 \\
-2 & 0 & -1 & 0 & 0 & 0 \\
0 & -1 & 0 & -2 & -2 & 0 \\
0 & 0 & -1 & 2 & 0 & -1 \\
0 & 0 & -1 & 0 & 2 & 0 \\
0 & 0 & 0 & -1 & 0 & 2
\end{array}\right) \text {, } \\
& \text { 14) }\left(\begin{array}{cccccc}
0 & -1 & 0 & 0 & 0 & 0 \\
-1 & 2 & -1 & 0 & 0 & 0 \\
0 & -1 & 2 & 0 & -1 & 0 \\
0 & 0 & 0 & 2 & -1 & -1 \\
0 & 0 & -1 & -1 & 0 & 0 \\
0 & 0 & 0 & -1 & 0 & 2
\end{array}\right) \text {, } \\
& \left(\begin{array}{cccccc}
2 & -1 & 0 & 0 & 0 & 0 \\
-1 & 2 & -1 & 0 & 0 & 0 \\
0 & -2 & 0 & -1 & 0 & 0 \\
0 & 0 & -1 & 0 & -2 & -2 \\
0 & 0 & 0 & -1 & 2 & 0 \\
0 & 0 & 0 & -1 & 0 & 0
\end{array}\right),
\end{aligned}
$$


$\left.\left.16)\left(\begin{array}{cccccc}2 & -1 & 0 & 0 & 0 & 0 \\ -2 & 0 & -1 & 0 & 0 & 0 \\ 0 & -1 & 0 & 0 & -2 & 0 \\ 0 & 0 & 0 & 2 & -1 & -1 \\ 0 & 0 & -1 & -1 & 0 & 0 \\ 0 & 0 & 0 & -1 & 0 & 2\end{array}\right), 17\right)\left(\begin{array}{cccccc}2 & -1 & 0 & 0 & 0 & 0 \\ -1 & 0 & -2 & 0 & 0 & 0 \\ 0 & -1 & 2 & -1 & -1 & 0 \\ 0 & 0 & -1 & 2 & 0 & -1 \\ 0 & 0 & -1 & 0 & 2 & 0 \\ 0 & 0 & 0 & -1 & 0 & 2\end{array}\right), 18\right)\left(\begin{array}{cccccc}2 & -1 & 0 & 0 & 0 & 0 \\ -1 & 2 & -1 & 0 & 0 & 0 \\ 0 & -1 & 2 & -1 & 0 & 0 \\ 0 & 0 & -2 & 0 & -1 & -1 \\ 0 & 0 & 0 & -1 & 0 & -1 \\ 0 & 0 & 0 & -1 & -1 & 2\end{array}\right)$,

$\left.\left.19)\left(\begin{array}{cccccc}2 & -1 & 0 & 0 & 0 & 0 \\ -1 & 2 & -1 & 0 & 0 & 0 \\ 0 & -2 & 0 & 0 & -1 & 0 \\ 0 & 0 & 0 & 2 & -1 & -1 \\ 0 & 0 & -1 & -2 & 2 & 0 \\ 0 & 0 & 0 & -1 & 0 & 2\end{array}\right), 20\right)\left(\begin{array}{cccccc}2 & -1 & 0 & 0 & 0 & 0 \\ -1 & 2 & -1 & 0 & 0 & 0 \\ 0 & -1 & 2 & -1 & 0 & 0 \\ 0 & 0 & -1 & 2 & -1 & 0 \\ 0 & 0 & 0 & -1 & 0 & -1 \\ 0 & 0 & 0 & 0 & -1 & 0\end{array}\right), 21\right)\left(\begin{array}{cccccc}2 & -1 & 0 & 0 & 0 & 0 \\ -1 & 2 & -1 & 0 & 0 & 0 \\ 0 & -1 & 2 & -1 & 0 & 0 \\ 0 & 0 & -1 & 2 & -1 & 0 \\ 0 & 0 & 0 & -2 & 2 & -1 \\ 0 & 0 & 0 & 0 & -1 & 0\end{array}\right)$.

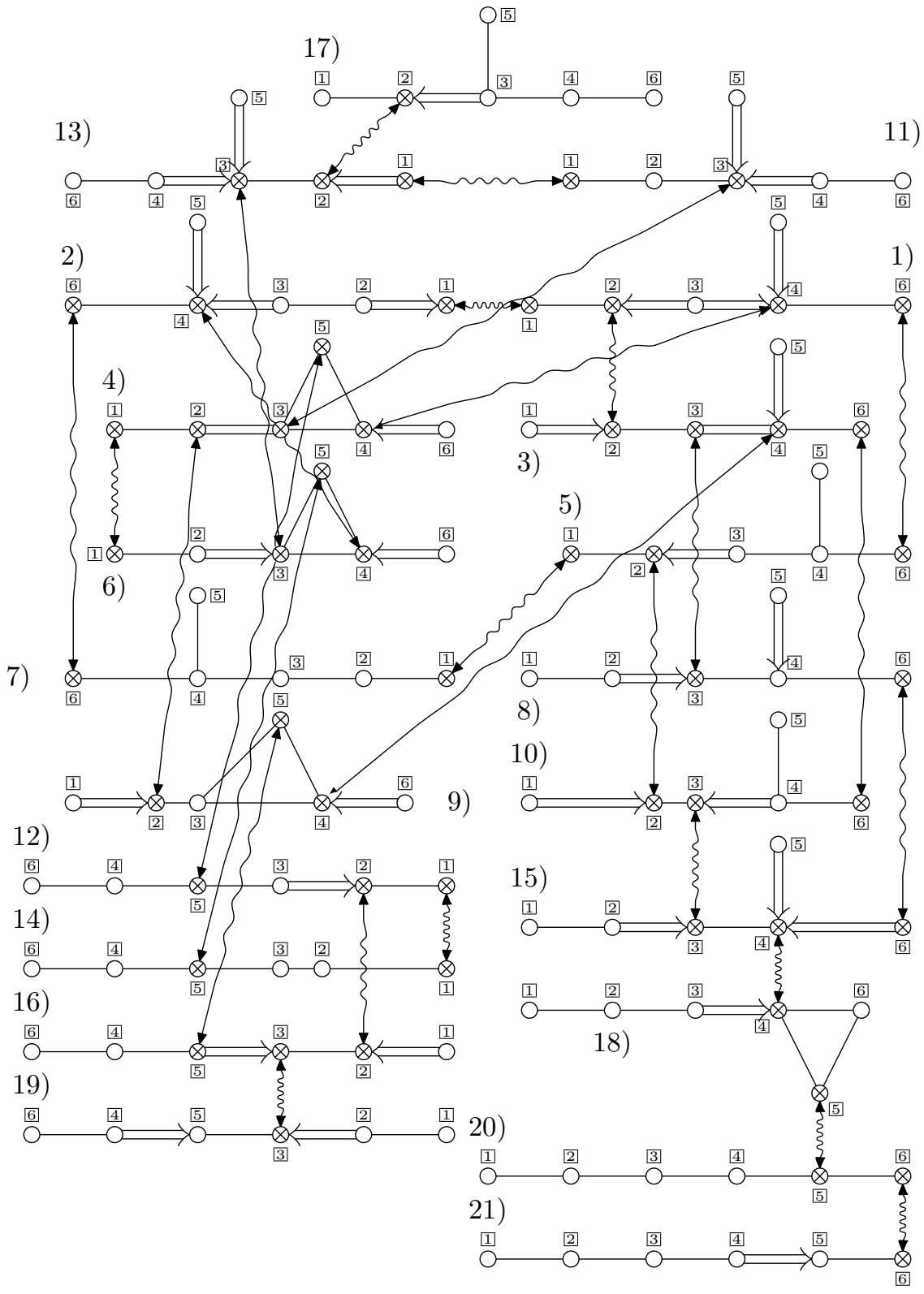


12.1.10 $\mathfrak{g}(8,6)$ of $\operatorname{sdim}=133 \mid 56$

We have $\mathfrak{g}(8,6)_{\overline{0}}=\mathfrak{e}(7)$ and $\mathfrak{g}(8,6)_{\overline{1}}=R\left(\pi_{1}\right)$.

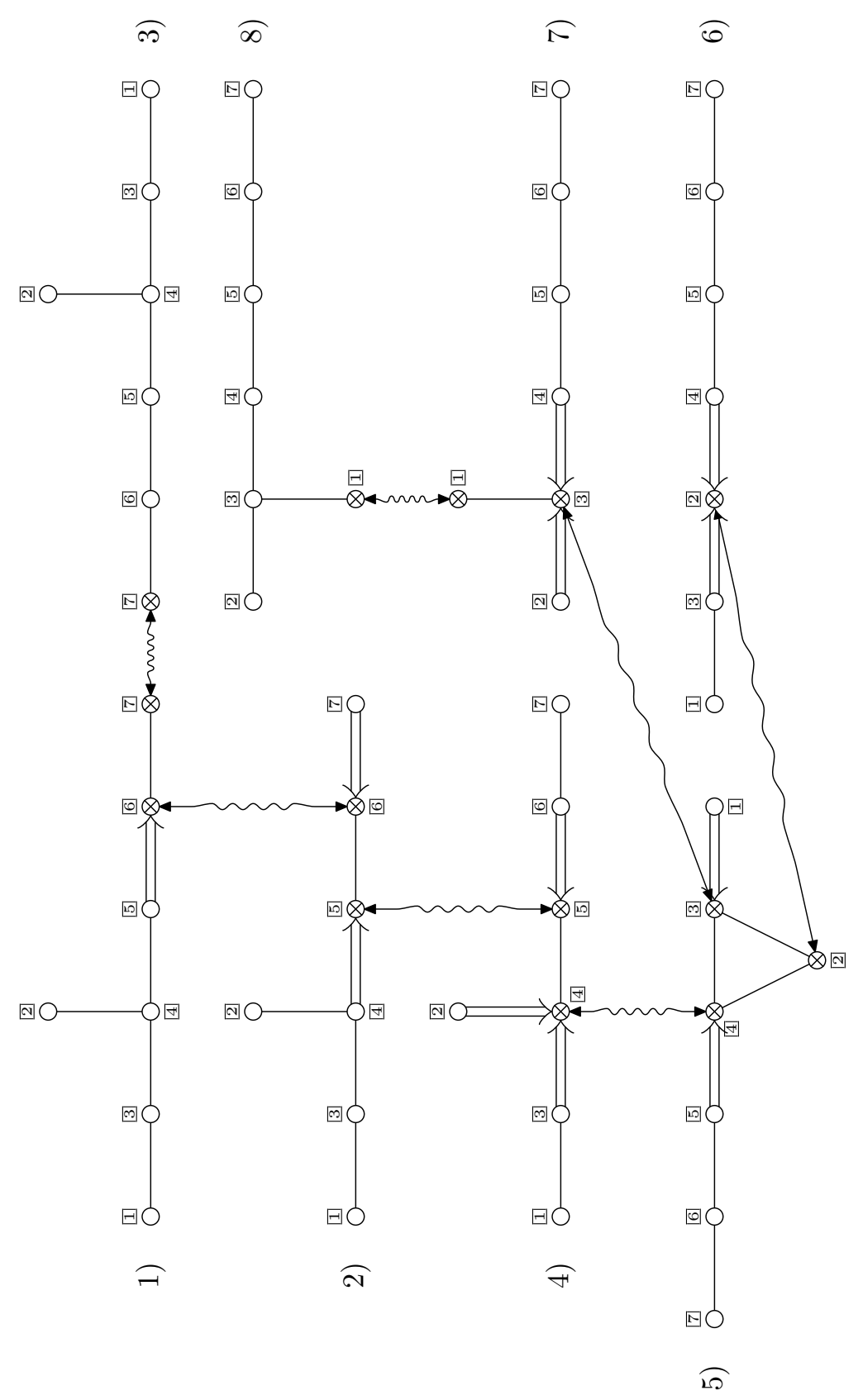

$$
\left(\begin{array}{ccccccc}
- & - & - & - & - & 2 & 3 \\
- & - & - & - & 4 & 1 & - \\
- & - & - & - & - & - & 1 \\
- & - & - & 5 & 2 & - & - \\
- & 6 & 7 & 4 & - & - & - \\
- & 5 & - & - & - & - & - \\
8 & - & 5 & - & - & - & - \\
7 & - & - & - & - & - & -
\end{array}\right),
$$




$$
\begin{aligned}
& \left.1)\left(\begin{array}{ccccccc}
2 & 0 & -1 & 0 & 0 & 0 & 0 \\
0 & 2 & 0 & -1 & 0 & 0 & 0 \\
-1 & 0 & 2 & -1 & 0 & 0 & 0 \\
0 & -1 & -1 & 2 & -1 & 0 & 0 \\
0 & 0 & 0 & -1 & 2 & -1 & 0 \\
0 & 0 & 0 & 0 & -2 & 0 & -1 \\
0 & 0 & 0 & 0 & 0 & -1 & 0
\end{array}\right), \quad 2\right)\left(\begin{array}{ccccccc}
2 & 0 & -1 & 0 & 0 & 0 & 0 \\
0 & 2 & 0 & -1 & 0 & 0 & 0 \\
-1 & 0 & 2 & -1 & 0 & 0 & 0 \\
0 & -1 & -1 & 2 & -1 & 0 & 0 \\
0 & 0 & 0 & -2 & 0 & -1 & 0 \\
0 & 0 & 0 & 0 & -1 & 0 & -2 \\
0 & 0 & 0 & 0 & 0 & -1 & 2
\end{array}\right) \text {, } \\
& \left.3)\left(\begin{array}{ccccccc}
2 & 0 & -1 & 0 & 0 & 0 & 0 \\
0 & 2 & 0 & -1 & 0 & 0 & 0 \\
-1 & 0 & 2 & -1 & 0 & 0 & 0 \\
0 & -1 & -1 & 2 & -1 & 0 & 0 \\
0 & 0 & 0 & -1 & 2 & -1 & 0 \\
0 & 0 & 0 & 0 & -1 & 2 & -1 \\
0 & 0 & 0 & 0 & 0 & -1 & 0
\end{array}\right), \quad 4\right)\left(\begin{array}{ccccccc}
2 & 0 & -1 & 0 & 0 & 0 & 0 \\
0 & 2 & 0 & -1 & 0 & 0 & 0 \\
-1 & 0 & 2 & -1 & 0 & 0 & 0 \\
0 & -2 & -2 & 0 & -1 & 0 & 0 \\
0 & 0 & 0 & -1 & 0 & -2 & 0 \\
0 & 0 & 0 & 0 & -1 & 2 & -1 \\
0 & 0 & 0 & 0 & 0 & -1 & 2
\end{array}\right), \\
& \left.5)\left(\begin{array}{ccccccc}
2 & 0 & -1 & 0 & 0 & 0 & 0 \\
0 & 0 & -1 & -1 & 0 & 0 & 0 \\
-2 & -1 & 0 & -1 & 0 & 0 & 0 \\
0 & -1 & -1 & 0 & -2 & 0 & 0 \\
0 & 0 & 0 & -1 & 2 & -1 & 0 \\
0 & 0 & 0 & 0 & -1 & 2 & -1 \\
0 & 0 & 0 & 0 & 0 & -1 & 2
\end{array}\right), \quad 6\right)\left(\begin{array}{ccccccc}
2 & 0 & -1 & 0 & 0 & 0 & 0 \\
0 & 0 & -2 & -2 & 0 & 0 & 0 \\
-1 & -1 & 2 & 0 & 0 & 0 & 0 \\
0 & -1 & 0 & 2 & -1 & 0 & 0 \\
0 & 0 & 0 & -1 & 2 & -1 & 0 \\
0 & 0 & 0 & 0 & -1 & 2 & -1 \\
0 & 0 & 0 & 0 & 0 & -1 & 2
\end{array}\right) \text {, } \\
& \left.7)\left(\begin{array}{ccccccc}
0 & 0 & -1 & 0 & 0 & 0 & 0 \\
0 & 2 & -1 & 0 & 0 & 0 & 0 \\
-1 & -2 & 0 & -2 & 0 & 0 & 0 \\
0 & 0 & -1 & 2 & -1 & 0 & 0 \\
0 & 0 & 0 & -1 & 2 & -1 & 0 \\
0 & 0 & 0 & 0 & -1 & 2 & -1 \\
0 & 0 & 0 & 0 & 0 & -1 & 2
\end{array}\right), \quad 8\right)\left(\begin{array}{ccccccc}
0 & 0 & -1 & 0 & 0 & 0 & 0 \\
0 & 2 & -1 & 0 & 0 & 0 & 0 \\
-1 & -1 & 2 & -1 & 0 & 0 & 0 \\
0 & 0 & -1 & 2 & -1 & 0 & 0 \\
0 & 0 & 0 & -1 & 2 & -1 & 0 \\
0 & 0 & 0 & 0 & -1 & 2 & -1 \\
0 & 0 & 0 & 0 & 0 & -1 & 2
\end{array}\right) .
\end{aligned}
$$

\subsection{The Elduque superalgebra $\mathfrak{e l}(5 ; 3)$ : Systems of simple roots}

Its superdimension is $39 \mid 32$; the even part is $\mathfrak{e l}(5 ; 3)_{\overline{0}}=\mathfrak{o}(9) \oplus \mathfrak{s l}(2)$ and its odd part is irreducible: $\mathfrak{e l}(5 ; 3)_{\overline{1}}=R\left(\pi_{4}\right) \otimes \mathrm{id}$.

The following are all its Cartan matrices:

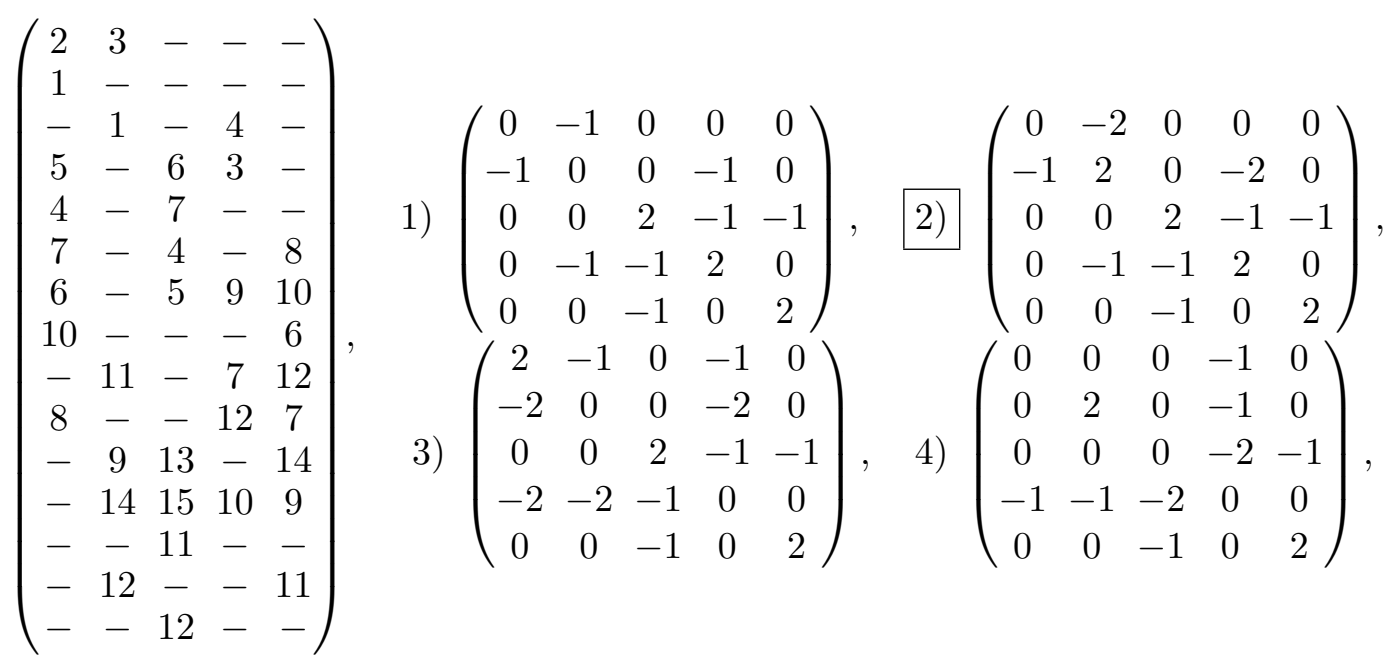




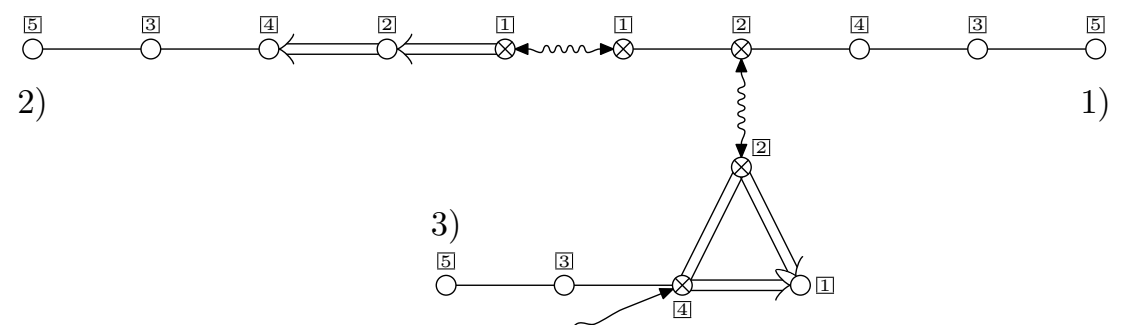

5)

4)

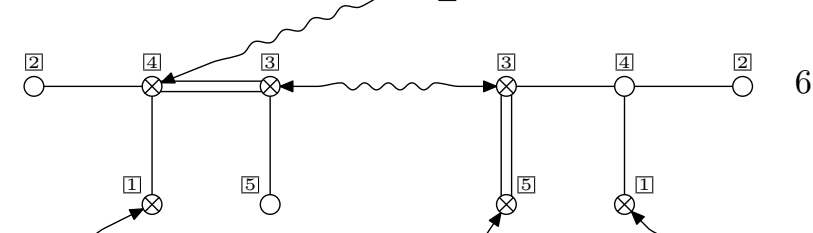

8)

$\frac{8}{11}$

6)

12)

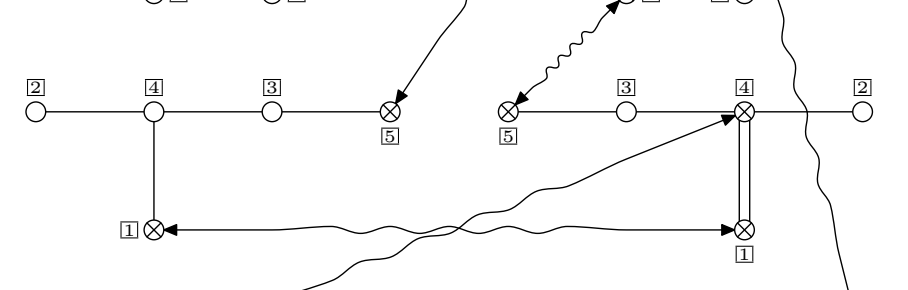

10)

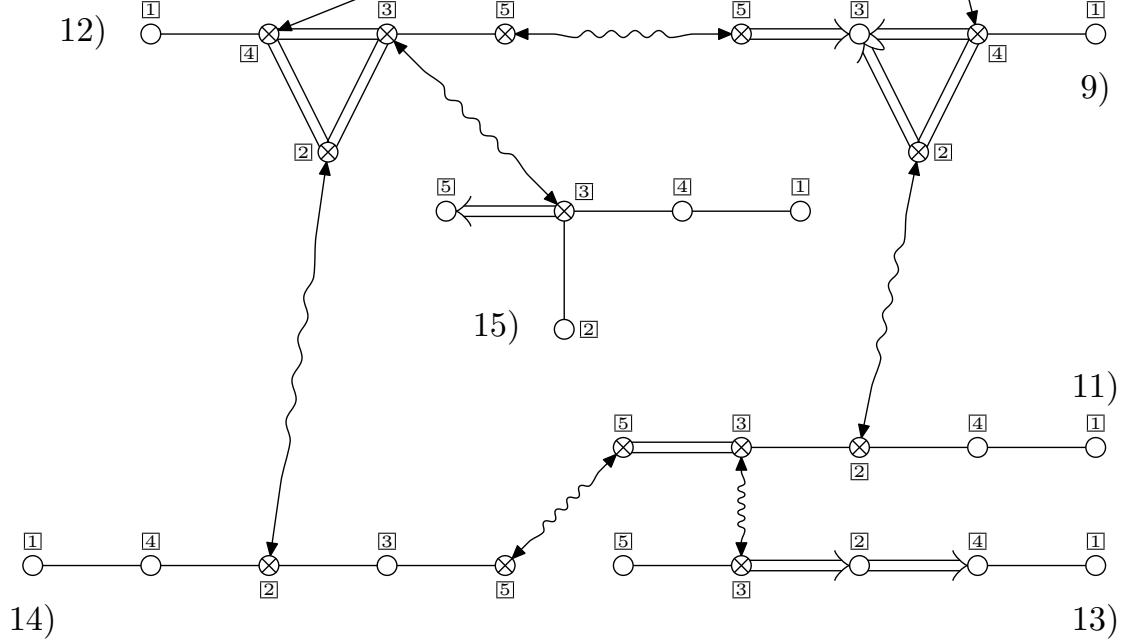

$\left.\left.5)\left(\begin{array}{ccccc}0 & 0 & 0 & -2 & 0 \\ 0 & 2 & 0 & -1 & 0 \\ 0 & 0 & 0 & -2 & -1 \\ -1 & -2 & -1 & 2 & 0 \\ 0 & 0 & -1 & 0 & 2\end{array}\right), \quad 6\right)\left(\begin{array}{ccccc}0 & 0 & 0 & -1 & 0 \\ 0 & 2 & 0 & -1 & 0 \\ 0 & 0 & 0 & -1 & -2 \\ -1 & -1 & -1 & 2 & 0 \\ 0 & 0 & -2 & 0 & 0\end{array}\right), \quad 7\right)\left(\begin{array}{ccccc}0 & 0 & 0 & -2 & 0 \\ 0 & 2 & 0 & -1 & 0 \\ 0 & 0 & 0 & -1 & -2 \\ -2 & -1 & -1 & 0 & 0 \\ 0 & 0 & -2 & 0 & 0\end{array}\right)$,

$\left.\left.8)\left(\begin{array}{ccccc}0 & 0 & 0 & -1 & 0 \\ 0 & 2 & 0 & -1 & 0 \\ 0 & 0 & 2 & -1 & -1 \\ -1 & -1 & -1 & 2 & 0 \\ 0 & 0 & -1 & 0 & 0\end{array}\right), \quad 9\right)\left(\begin{array}{ccccc}2 & 0 & 0 & -1 & 0 \\ 0 & 0 & -2 & -2 & 0 \\ 0 & -1 & 2 & -1 & -1 \\ -1 & -2 & -2 & 0 & 0 \\ 0 & 0 & -2 & 0 & 0\end{array}\right), \quad 10\right)\left(\begin{array}{ccccc}0 & 0 & 0 & -2 & 0 \\ 0 & 2 & 0 & -1 & 0 \\ 0 & 0 & 2 & -1 & -1 \\ -2 & -1 & -1 & 0 & 0 \\ 0 & 0 & -1 & 0 & 0\end{array}\right)$, 

11) $\left(\begin{array}{ccccc}2 & 0 & 0 & -1 & 0 \\ 0 & 0 & -1 & -1 & 0 \\ 0 & -1 & 0 & 0 & -2 \\ -1 & -1 & 0 & 2 & 0 \\ 0 & 0 & -2 & 0 & 0\end{array}\right)$,
12) $\left(\begin{array}{ccccc}2 & 0 & 0 & -1 & 0 \\ 0 & 0 & -2 & -2 & 0 \\ 0 & -2 & 0 & -2 & -1 \\ -1 & -2 & -2 & 0 & 0 \\ 0 & 0 & -1 & 0 & 0\end{array}\right)$,
$13)\left(\begin{array}{ccccc}2 & 0 & 0 & -1 & 0 \\ 0 & 2 & -1 & -2 & 0 \\ 0 & -2 & 0 & 0 & -1 \\ -1 & -1 & 0 & 2 & 0 \\ 0 & 0 & -1 & 0 & 2\end{array}\right)$
14) $\left(\begin{array}{ccccc}2 & 0 & 0 & -1 & 0 \\ 0 & 0 & -1 & -1 & 0 \\ 0 & -1 & 2 & 0 & -1 \\ -1 & -1 & 0 & 2 & 0 \\ 0 & 0 & -1 & 0 & 0\end{array}\right)$,
$15)\left(\begin{array}{ccccc}2 & 0 & 0 & -1 & 0 \\ 0 & 2 & -1 & 0 & 0 \\ 0 & -1 & 0 & -1 & -2 \\ -1 & 0 & -1 & 2 & 0 \\ 0 & 0 & -1 & 0 & 2\end{array}\right)$.

\section{The answer: The case where $p=2$}

Simple Lie algebras:

1) The Lie algebras obtained from their Cartan matrices by reducing modulo 2 (for $\mathfrak{o}(2 n+1)$ one has, first of all, to divide the last row by 2 in order to adequately normalize CM). We thus get:

the CM versions of $\mathfrak{s l}$, namely: $\mathfrak{s l}(2 n+1)$, and $\mathfrak{g l}(2 n)$ whose "simple core" is $\mathfrak{p s l}(2 n)$; in the "second" integer basis of $\mathfrak{g}(2)$ given in [24, p. 346], all structure constants are integer and $\mathfrak{g}(2)$ becomes, after reduction modulo 2, a simple Lie algebra $\mathfrak{p s l}(4)$ (without Cartan matrix, as we know);

the "simple cores" of the orthogonal algebras, namely, of $\mathfrak{o}^{(1)}(2 n+1)$ and $\mathfrak{o c}(2 n)$; $\mathfrak{e}(6), \mathfrak{e}(7)^{(1)} / \mathfrak{c}, \mathfrak{e}(8) ;$

2) the Weisfeiler and Kac algebras $\mathfrak{w} \mathfrak{k}(3 ; a)^{(1)} / \mathfrak{c}$ and $\mathfrak{w} \mathfrak{k}(4 ; a)$.

Simple Lie superalgebras

In the list below the term "super version" of a Lie algebra $\mathfrak{g}(A)$ stands for a Lie superalgebra with the "same" root system as that of $\mathfrak{g}(A)$ but with some of the simple roots considered odd.

1) The Lie superalgebras obtained from their $p=0$ analogs that have no -2 in off-diagonal slots of the Cartan matrix by reducing the structure constants modulo 2 (for $\mathfrak{o s p}(2 n+1 \mid 2 m$ ) one has, first of all, to divide the last row by 2 in order to normalize $\mathrm{CM}$ ), we thus get

the CM versions of $\mathfrak{s l}$, namely: either simple $\mathfrak{s l}(a \mid a+2 k+1)$ or $\mathfrak{g l}(a \mid 2 k+a)$ whose "simple core" is $\mathfrak{p s l}(a \mid a+2 k)$ for $a$ odd or $\mathfrak{p s l}(a \mid a+2 k)^{(1)}$ for $a$ even;

2) the ortho-orthogonal algebras, namely: $\mathfrak{o o}^{(1)}$ and ooc whose "simple cores" are described in Section 6;

3) $\mathfrak{b} \mathfrak{g l}(3 ; a)^{(1)} / \mathfrak{c}$ which is an analog of $\mathfrak{w} \mathfrak{k}(3 ; a)^{(1)} / \mathfrak{c}$ with the "same" Cartan matrices but different root systems;

4) the CM versions of periplectic algebras, namely: $\mathfrak{p e c}$; these are at the same time super versions of oc; their "simple cores" are described in Section 6;

5) a super version of $\mathfrak{w k}(4 ; a)$, namely: $\mathfrak{b g l}(4 ; a)$;

6) the super versions of $\mathfrak{e}(6)$, namely: $\mathfrak{e}(6,1), \mathfrak{e}(6,6)$;

7) the super versions of $\mathfrak{e}(7)$, namely: $\mathfrak{e}(7,1), \mathfrak{e}(7,6), \mathfrak{e}(7,7)$ whose "simple cores" are described in Section 13.1.5;

8) the super versions of $\mathfrak{e}(8)$, namely: $\mathfrak{e}(8,1), \mathfrak{e}(8,8)$. 


\subsection{On the structure of $\mathfrak{b} \mathfrak{g l}(3 ; \alpha), \mathfrak{b} \mathfrak{g l}(4 ; \alpha)$, and $\mathfrak{e}(a, b)$}

In this section we describe the even parts $\mathfrak{g}_{0}$ of the new Lie superalgebras $\mathfrak{g}=\mathfrak{g}(A)$ and their odd parts $\mathfrak{g}_{\overline{1}}$ as $\mathfrak{g}_{0}$-modules. SuperLie enumerates the elements of the Chevalley basis the $x_{i}$ (positive), starting with the generators, then their brackets, etc., and the $y_{i}$ are negative root vectors opposite to the $x_{i}$. Since the irreducible representations of the Lie algebras may have neither highest nor lowest weight, observe that the $\mathfrak{g}_{\overline{0}}$-modules $\mathfrak{g}_{\overline{1}}$ always have both highest and lowest weights.

\subsubsection{Notation $\mathfrak{A} \oplus_{\mathfrak{c}} \mathfrak{B}$ needed to describe $\mathfrak{b} \mathfrak{g l}(4 ; \alpha), \mathfrak{e}(6,6), \mathfrak{e}(7,6)$, and $\mathfrak{e}(8,1)$}

This notation describes the case where $\mathfrak{A}$ and $\mathfrak{B}$ are nontrivial central extensions of the Lie algebras $\mathfrak{a}$ and $\mathfrak{b}$, respectively, and $\mathfrak{A} \oplus_{c} \mathfrak{B}$ - a nontrivial central extension of $\mathfrak{a} \oplus \mathfrak{b}$ (or, perhaps, a more complicated $\mathfrak{a} \in \mathfrak{b}$ ) with 1-dimensional center spanned by $c$ - is such that the restriction of the extension of $\mathfrak{a} \oplus \mathfrak{b}$ to $\mathfrak{a}$ gives $\mathfrak{A}$ and that to $\mathfrak{b}$ gives $\mathfrak{B}$. (In other words, the situation resembles the (nontrivial) central extension of the Lie algebra of derivations of the loop algebra, namely, $\mathfrak{g} \otimes \mathbb{C}\left[t^{-1}, t\right] \notin \mathfrak{d} \mathfrak{e r}\left(\mathbb{C}\left[t^{-1}, t\right]\right)$, where one central element serves both central extensions: That of $\mathfrak{g} \otimes \mathbb{C}\left[t^{-1}, t\right]$ and of $\mathfrak{d e r}\left(\mathbb{C}\left[t^{-1}, t\right]\right)$.)

In these four cases, $\mathfrak{g}(A)_{\overline{0}}$ is of the form

$$
\mathfrak{g}(B) \oplus_{c} \mathfrak{h} \mathfrak{e i}(2) \simeq \mathfrak{g}(B) \oplus \operatorname{Span}\left(X^{+}, X^{-}\right),
$$

where the matrix $B$ is not invertible (so $\mathfrak{g}(B)$ has a grading element $d$ and a central element $c$ ), and where $X^{+}, X^{-}$and $c$ span the Heisenberg Lie algebra $\mathfrak{h e i}(2)$. The brackets are:

$$
\begin{aligned}
& {\left[\mathfrak{g}^{(1)}(B), X^{ \pm}\right]=0,} \\
& {\left[d, X^{ \pm}\right]=X^{ \pm}, \quad\left(\left[d, X^{ \pm}\right]=\alpha X^{ \pm} \text {for } \mathfrak{b} \mathfrak{g l}(4 ; \alpha)\right),} \\
& {\left[X^{+}, X^{-}\right]=c .}
\end{aligned}
$$

The odd part of $\mathfrak{g}(A)$ (at least in two of the four cases) consists of two copies of the same $\mathfrak{g}(B)$ module $N$, the operators $\operatorname{ad}_{X^{ \pm}}$permute these copies, and $\operatorname{ad}_{X^{ \pm}}^{2}=0$, so each of the operators maps one of the copies to the other, and this other copy to zero.

\subsection{2 $\mathfrak{b} \mathfrak{g l}(3 ; \alpha)$, where $\alpha \neq 0,1 ; \operatorname{sdim}=10 / 8 \mid 8$}

We consider the following Cartan matrix and the corresponding positive root vectors (odd | even)

$$
\left(\begin{array}{ccc}
0 & 1 & 0 \\
1 & \overline{0} & \alpha \\
0 & \alpha & \overline{0}
\end{array}\right) \quad \begin{aligned}
& x_{1} \mid x_{2}, \quad x_{3}, \\
& x_{4}=\left[x_{1}, x_{2}\right]\left|x_{5}=\left[x_{2}, x_{3}\right], \quad x_{6}=\left[x_{3},\left[x_{1}, x_{2}\right]\right],\right| \\
& x_{7}=\left[\left[x_{1}, x_{2}\right],\left[x_{2}, x_{3}\right]\right] \mid .
\end{aligned}
$$

Then $\mathfrak{g}_{\overline{0}} \simeq \mathfrak{g l}(3) \oplus \mathbb{K} Z$. The $\mathfrak{g}_{\overline{0}}$-module $\mathfrak{g}_{\overline{1}}$ is reducible, with the two highest weight vectors, $x_{7}$ and $y_{1}$. The Cartan subalgebra of $\mathfrak{g l}(3) \oplus \mathbb{K} Z$ is spanned by $\alpha h_{1}+h_{3}, h_{2}, h_{3}$ and $Z$. In this basis, the weight of $x_{7}$ is $(0,1+\alpha, 0,1)$. The weight of $y_{1}$ is $(0,1,0,1)$, if for the grading operator we take $(1,0,0) \in \mathfrak{g l}(3)$.

The lowest weight vectors of these modules are $x_{1}$ and $y_{7}$ and their weights are $(0,1,0,1)$ and $(0,1+\alpha, 0,1)$. The module generated by $x_{7}$ is $\operatorname{Span}\left\{x_{1}, x_{4}, x_{6}, x_{7}\right\}$. The module generated by $y_{1}$ is $\operatorname{Span}\left\{y_{1}, y_{4}, y_{6}, y_{7}\right\}$.

All inequivalent Cartan matrices are

$$
\left(\begin{array}{ccc}
d_{1} & \alpha & 1 \\
\alpha & d_{2} & 0 \\
1 & 0 & d_{3}
\end{array}\right), \quad\left(\begin{array}{ccc}
d_{1} & \alpha & 1+\alpha \\
\alpha & d_{2} & 1 \\
1+\alpha & 1 & d_{3}
\end{array}\right),
$$

where $\left(d_{1}, d_{2}, d_{3}\right)$ is any distribution of 0 's and $\overline{0}$ 's, except $(\overline{0}, \overline{0}, \overline{0})$. 


\subsection{3 $\mathfrak{b g l}(4 ; \alpha)$, where $\alpha \neq 0,1$, of $\operatorname{sdim}=18 \mid 16$}

We consider the following Cartan matrix and the corresponding positive root vectors (odd even)

$$
\begin{aligned}
& x_{1} \mid x_{2}, \quad x_{3}, \quad x_{4}, \\
& x_{5}=\left[x_{1}, x_{2}\right], \quad x_{6}=\left[x_{1}, x_{3}\right] \mid x_{7}=\left[x_{3}, x_{4}\right] \text {, } \\
& \left(\begin{array}{llll}
0 & \alpha & 1 & 0 \\
\alpha & 0 & 0 & 0
\end{array}\right) \quad x_{8}=\left[x_{3},\left[x_{1}, x_{2}\right]\right], \quad x_{9}=\left[x_{4},\left[x_{1}, x_{3}\right]\right] \\
& \begin{array}{cccc}
\alpha & \overline{0} & 0 & 0
\end{array} \quad x_{11}=\left[\left[x_{1}, x_{2}\right],\left[x_{3}, x_{4}\right]\right] \mid x_{10}=\left[\left[x_{1}, x_{2}\right],\left[x_{1}, x_{3}\right]\right] \\
& \begin{array}{llll}
1 & 0 & \overline{0} & 1
\end{array} \quad \mid x_{12}=\left[\left[x_{1}, x_{2}\right],\left[x_{4},\left[x_{1}, x_{3}\right]\right]\right] \text {, } \\
& \begin{array}{llll}
0 & 0 & 1 & \overline{0}
\end{array} \quad \mid x_{13}=\left[\left[x_{3},\left[x_{1}, x_{2}\right]\right],\left[x_{4},\left[x_{1}, x_{3}\right]\right]\right] \text {, } \\
& x_{14}=\left[\left[x_{4},\left[x_{1}, x_{3}\right]\right],\left[\left[x_{1}, x_{2}\right],\left[x_{1}, x_{3}\right]\right]\right] \mid \\
& x_{15}=\left[\left[\left[x_{1}, x_{2}\right],\left[x_{1}, x_{3}\right]\right],\left[\left[x_{1}, x_{2}\right],\left[x_{3}, x_{4}\right]\right]\right] \mid \text {. }
\end{aligned}
$$

In this case $\mathfrak{g}_{\overline{0}} \simeq \mathfrak{g l}(4) \oplus_{c} \mathfrak{h} \mathfrak{e i}(2)$ see Section 13.1 .1 with commutation relations (13.1). The $\mathfrak{g}_{\overline{0}}$-module $\mathfrak{g}_{\overline{1}}$ is irreducible: $\mathfrak{g}_{\overline{1}} \simeq N \otimes \mathrm{id}$, where id is the standard 2-dimensional $\mathfrak{h} \mathfrak{e i}(2)$-module and $N$ is an 8-dimensional $\mathfrak{g l}(4)$-module.

The highest weight vector $x_{15}$ has weight $(\alpha, 0,0,0, \alpha)$ with respect to

$$
c=h_{2}, \quad d=h_{1}, \quad H_{1}=h_{3}, \quad H_{2}=h_{3}, \quad H_{3}=h_{2}+h_{3},
$$

where the $h_{i}$ 's are the Chevalley generators of the Cartan subalgebra of $\mathfrak{b g r}(4 ; \alpha)$. The lowest weight vector is $y_{15}$ of the same weight as $x_{15}$.

All inequivalent Cartan matrices of $\mathfrak{b} \mathfrak{g l}(4 ; \alpha)$ are

$$
\left(\begin{array}{cccc}
d_{1} & \alpha & 0 & 0 \\
\alpha & d_{2} & 1 & 0 \\
0 & 1 & d_{3} & 1 \\
0 & 0 & 1 & d_{4}
\end{array}\right), \quad\left(\begin{array}{cccc}
d_{1} & 1 & 1+\alpha & 0 \\
1 & d_{2} & \alpha & 0 \\
\alpha+1 & \alpha & d_{3} & \alpha \\
0 & 0 & \alpha & d_{4}
\end{array}\right), \quad\left(\begin{array}{cccc}
d_{1} & \alpha & 0 & 0 \\
\alpha & d_{2} & \alpha+1 & 0 \\
0 & \alpha+1 & d_{3} & 1 \\
0 & 0 & 1 & d_{4}
\end{array}\right)
$$

where $\left\{d_{1}, d_{2}, d_{3}, d_{4}\right\}$ is any distribution of 0 's and $\overline{0}$ 's, except $\{\overline{0}, \overline{0}, \overline{0}, \overline{0}\}$.

13.1.4. Proposition (cf. (9.5) and (9.2)).

1) We have

$$
\begin{aligned}
& \mathfrak{b g h}(3 ; a) \simeq \mathfrak{b} \mathfrak{g l}\left(3 ; a^{\prime}\right) \Longleftrightarrow a^{\prime}=\frac{\alpha a+\beta}{\gamma a+\delta}, \quad \text { where }\left(\begin{array}{ll}
\alpha & \beta \\
\gamma & \delta
\end{array}\right) \in \operatorname{SL}(2 ; \mathbb{Z} / 2) \\
& \mathfrak{b g l}(4 ; a) \simeq \mathfrak{b} \mathfrak{g l}\left(4 ; a^{\prime}\right) \Longleftrightarrow a^{\prime}=\frac{1}{a} .
\end{aligned}
$$

2) The 2|4-structures on $\mathfrak{b g l}(3 ; a)$ and $\mathfrak{b} \mathfrak{g l}(4 ; a)$ are given by the same formulas (9.6), (9.7), (9.8) as for $\mathfrak{w k}(3 ; a)$ and $\mathfrak{w k}(4 ; a)$ with the following amendment:

$$
\begin{array}{ll}
\left(e_{\alpha}^{ \pm}\right)^{[2]}=0 & \text { for any root vector } e_{\alpha} \text { even } \\
\left(e_{\alpha}^{ \pm}\right)^{[4]}=\left(\left(e_{\alpha}^{ \pm}\right)^{2}\right)^{[2]} & \text { for any root vector } e_{\alpha} \text { odd } .
\end{array}
$$

\subsubsection{The e-type superalgebras}

Notation. The $\mathfrak{e}$-type superalgebras will be denoted by (one of) their simplest Dynkin diagrams, i.e., $\mathfrak{e}(n, i)$ denotes the Lie superalgebra whose diagram is of the same shape as that of the Lie algebra $\mathfrak{e}(n)$ but with the only - $i$ th - node $\otimes$. This, and other "simplest", Cartan matrices are boxed. We enumerate the nodes of the Dynkin diagram of $\mathfrak{e}(n)$ as in $[8,41]$ : We first enumerate the nodes in the row corresponding to $\mathfrak{s l}(n)$ (from the end-point of the "longest" twig towards the branch point and further on along the second long twig), and the $n$th node is the end-point of the shortest "twig". 
$\mathfrak{e}(\mathbf{6}, \mathbf{1}) \simeq \mathfrak{e}(\mathbf{6}, \mathbf{5})$ of $\operatorname{sdim}=\mathbf{4 6} \mid \mathbf{3 2}$. We have $\mathfrak{g}_{\overline{0}} \simeq \mathfrak{o c}(2 ; 10) \oplus \mathbb{K} Z$ and $\mathfrak{g}_{\overline{1}}$ is a reducible module of the form $R\left(\pi_{4}\right) \oplus R\left(\pi_{5}\right)$ with the two highest weight vectors

$$
x_{36}=\left[\left[\left[x_{4}, x_{5}\right],\left[x_{6},\left[x_{2}, x_{3}\right]\right]\right],\left[\left[x_{3},\left[x_{1}, x_{2}\right]\right],\left[x_{6},\left[x_{3}, x_{4}\right]\right]\right]\right]
$$

and $y_{5}$. Denote the basis elements of the Cartan subalgebra by $Z, h_{1}, h_{2}, h_{3}, h_{4}, h_{6}$. The weights of $x_{36}$ and $y_{5}$ are respectively, $(0,0,0,0,0,1)$ and $(0,0,0,0,1,0)$. The module generated by $x_{36}$ gives all odd positive roots and the module generated by $y_{5}$ gives all odd negative roots.

$\mathfrak{e}(\mathbf{6}, \mathbf{6})$ of $\operatorname{sdim}=\mathbf{3 8} \mid \mathbf{4 0}$. In this case, $\mathfrak{g}(B) \simeq \mathfrak{g l}(6)$, see Section 13.1.1. The module $\mathfrak{g}_{\overline{1}}$ is irreducible with the highest weight vector

$$
x_{35}=\left[\left[\left[x_{3}, x_{6}\right],\left[x_{4},\left[x_{2}, x_{3}\right]\right]\right],\left[\left[x_{4}, x_{5}\right],\left[x_{3},\left[x_{1}, x_{2}\right]\right]\right]\right] \text { of weight }(0,0,1,0,0,1) .
$$

We consider the highest weight with respect to the elements

$$
h_{1}:=E_{11}-E_{22}, \ldots, h_{5}:=E_{55}-E_{66}, h_{6}:=E_{11}+E_{22}+E_{33}
$$

in $\mathfrak{g l}(6)$. We can equally well set

$$
h_{6}:=E_{11}+E_{22}+E_{33}+a c \text { for any } a \in \mathbb{K}, \text { where } c \text { is the non-zero central element of } \mathfrak{g}(B)
$$

but in our choice of $h_{6}=E_{11}+E_{22}+E_{33}$, we have $M=\bigwedge^{3}$ (id), as a $\mathfrak{g l}(6)$-module (note that to write $M=R\left(\pi_{3}\right)$ is not enough since this only describes $M$ as an $\mathfrak{s l}(6)$-module).

$\mathfrak{e}(\mathbf{7}, \mathbf{1})$ of $\operatorname{sdim}=\mathbf{8 0} / \mathbf{7 8} \mid \mathbf{5 4}$. Since the Cartan matrix of this Lie superalgebra is of rank 6 , a grading operator $d_{1}$ should be (and is) added. Now if we take $d_{1}=(1,0,0,0,0,0,0)$, then $\mathfrak{g}_{\overline{0}} \simeq(\mathfrak{e}(6) \oplus \mathbb{K} z) \oplus \mathbb{K} I_{0}$. The Cartan subalgebra is spanned by $h_{1}+h_{3}+h_{7}, h_{2}, h_{3}, h_{4}, h_{5}, h_{6}$, $h_{7}$ and $d_{1}$. We see that $\mathfrak{g}_{\overline{1}}$ has the two highest weight vectors:

$$
x_{63}=\left[\left[\left[\left[x_{2}, x_{3}\right],\left[x_{4}, x_{7}\right]\right],\left[\left[x_{3}, x_{4}\right],\left[x_{5}, x_{6}\right]\right]\right],\left[\left[\left[x_{4}, x_{7}\right],\left[x_{5}, x_{6}\right]\right],\left[\left[x_{4}, x_{5}\right],\left[x_{3},\left[x_{1}, x_{2}\right]\right]\right]\right]\right]
$$

and $y_{1}$. Their respective weights (if we take $\left.d_{1}=(1,0,0,0,0,0,0)\right)$ are $(0,0,0,0,0,1,0,1)$ and $(0,1,0,0,0,0,0,0)$. The module generated by $x_{63}$ gives all odd positive roots and the module generated by $y_{1}$ gives all odd negative roots.

$\mathfrak{e}(\mathbf{7}, \mathbf{6})$ of $\operatorname{sdim}=\mathbf{7 0 / 6 8} \mid \mathbf{6 4}$. We are in the same situation as before (Section 13.1.1). We have $\mathfrak{g}(B) \simeq \mathfrak{o c}(1 ; 12) \in \mathbb{K} I_{0}$. Note that in this case $\operatorname{size}(B)-\operatorname{rk}(B)=2$, so the center of $\mathfrak{g}(B)$ is 2-dimensional, and $\operatorname{dim} \mathfrak{g}(B)-\operatorname{dim} \mathfrak{g}^{(1)}(B)=2$. So we should be a bit more specific than in (13.1); namely, we have

$$
\begin{aligned}
& {\left[\mathfrak{o c}(1 ; 12), X^{ \pm}\right]=0,} \\
& {\left[I_{0}, X^{ \pm}\right]=X^{ \pm},} \\
& {\left[X^{+}, X^{-}\right]=h_{1}+h_{3}+h_{5} \quad\left(\text { which corresponds to } 1_{12} \text { in } \mathfrak{o c}(1 ; 12)\right) .}
\end{aligned}
$$

The module $\mathfrak{g}_{\overline{1}}$ is irreducible with the highest weight vector

$$
x_{62}=\left[\left[\left[x_{7},\left[x_{5},\left[x_{3}, x_{4}\right]\right]\right],\left[\left[x_{1}, x_{2}\right],\left[x_{3}, x_{4}\right]\right]\right],\left[\left[\left[x_{2}, x_{3}\right],\left[x_{4}, x_{5}\right]\right],\left[\left[x_{4}, x_{7}\right],\left[x_{5}, x_{6}\right]\right]\right]\right] .
$$

The Cartan subalgebra is spanned by $h_{1}+h_{3}+h_{5}, h_{1}, h_{2}, h_{3}, h_{4}, h_{7}$ and also $h_{6}$ and $d_{1}$. The weight of $x_{62}$ is $(1,0,0,0,0,0,1,0)$. The highest weight vector of $\mathfrak{g}_{1}$ is the highest weight vector of one of the copies of the $\mathfrak{g}(B)$-module $N$, see Section 13.1.1, so the highest weight of $N$ is the same as the highest weight of $\mathfrak{g}_{\overline{1}}$. (Of course, this is true for the other two similar cases as well; in the case of $\mathfrak{e}(6,6)$, we used Lebedev's choice - another basis of $\mathfrak{h}$ - and expressed the weight with respect to it.) 
$\mathfrak{e}(\mathbf{7}, 7)$ of $\operatorname{sdim}=64 / 62 \mid 70$. Since the Cartan matrix of this Lie superalgebra is of rank 6 , a grading operator $d_{1}$ should be (and is) added. Then $\mathfrak{g}_{\overline{0}} \simeq \mathfrak{g l}(8)$. The module $\mathfrak{g}_{\overline{1}}$ has the two highest weight vectors:

$$
x_{58}=\left[\left[\left[x_{3},\left[x_{1}, x_{2}\right]\right],\left[x_{6},\left[x_{4}, x_{5}\right]\right]\right],\left[\left[x_{7},\left[x_{3}, x_{4}\right]\right],\left[\left[x_{2}, x_{3}\right],\left[x_{4}, x_{5}\right]\right]\right]\right]
$$

and $y_{7}$. The Cartan subalgebra is spanned by $h_{1}, h_{2}, h_{3}, h_{4}, h_{5}, h_{6}$ and also $h_{1}+h_{3}+h_{7}$ and $d_{1}$. The weight of $x_{58}$ with respect to these elements of the Cartan subalgebra is $(0,0,1,0,0,0,0,1)$ and the weight of $y_{7}$ is $(0,0,0,1,0,0,0,1)$. The module generated by $x_{58}$ gives all odd positive roots and the module generated by $y_{7}$ gives all odd negative roots.

$\mathfrak{e}(\mathbf{8}, \mathbf{1})$ of $\operatorname{sdim}=\mathbf{1 3 6} \mid \mathbf{1 1 2}$. We have (cf. Section 13.1.1) $\mathfrak{g}(B) \simeq \mathfrak{e}(7)$. (Recall that, in our notation, $\mathfrak{e}(7)^{(1)}$ has a center but not the grading operator, see Section "Warning" 4.1.) The Cartan subalgebra is spanned by $h_{2}+h_{4}+h_{8}$ and $h_{1}, h_{2}, h_{3}, h_{4}, h_{5}, h_{6}, h_{7}$. The $\mathfrak{g}_{0}$-module $\mathfrak{g}_{\overline{1}}$ is irreducible with the highest weight vector:

$$
\begin{aligned}
x_{119}= & {\left[\left[\left[\left[x_{4},\left[x_{2}, x_{3}\right]\right],\left[\left[x_{5}, x_{8}\right],\left[x_{6}, x_{7}\right]\right]\right],\left[\left[x_{8},\left[x_{4}, x_{5}\right]\right],\left[\left[x_{3}, x_{4}\right],\left[x_{5}, x_{6}\right]\right]\right]\right],\right.} \\
& {\left.\left[\left[\left[x_{7},\left[x_{5}, x_{6}\right]\right],\left[\left[x_{1}, x_{2}\right],\left[x_{3}, x_{4}\right]\right]\right],\left[\left[x_{8},\left[x_{5}, x_{6}\right]\right],\left[\left[x_{2}, x_{3}\right],\left[x_{4}, x_{5}\right]\right]\right]\right]\right] }
\end{aligned}
$$

of weight $(1,1,0,0,0,0,0,1)$ and one lowest weight vector $y_{119}$ whose expression is as above the $x$ 's changed by the $y$ 's, of the same weight as that of $x_{119}$. (Again, the highest weight of the $\mathfrak{g}(B)$-module $N$, see Section 13.1.1, is the same as the highest weight of $\mathfrak{g}_{\overline{1}}$.)

$\mathfrak{e}(\mathbf{8}, \mathbf{8})$ of sdim $=\mathbf{1 2 0} \mid \mathbf{1 2 8}$. In the $\mathbb{Z}$-grading with the 1 st $\mathrm{CM}$ with $\operatorname{deg} e_{8}^{ \pm}= \pm 1$ and $\operatorname{deg} e_{i}^{ \pm}=$ 0 for $i \neq 8$, we have $\mathfrak{g}_{0}=\mathfrak{g l}(8)=\mathfrak{g l}(V)$. There are different isomorphisms between $\mathfrak{g}_{0}$ and $\mathfrak{g l}(8)$; using the one where $h_{i}=E_{i, i}+E_{i+1, i+1}$ for all $i=1, \ldots, 7$, and $h_{8}=E_{6,6}+E_{7,7}+E_{8,8}$, we see that, as modules over $\mathfrak{g l}(V)$,

$$
\begin{array}{lll}
\mathfrak{g}_{1}=\bigwedge^{5} V^{*}, & \mathfrak{g}_{2}=\bigwedge^{6} V, & \mathfrak{g}_{3}=V, \\
\mathfrak{g}_{-1}=\bigwedge^{5} V, & \mathfrak{g}_{-2}=\bigwedge^{6} V^{*}, & \mathfrak{g}_{-3}=V^{*}
\end{array}
$$

We can also set $h_{8}=E_{1,1}+E_{2,2}+E_{3,3}+E_{4,4}+E_{5,5}$. Then we get

$$
\begin{array}{lll}
\mathfrak{g}_{1}=\bigwedge^{3} V, & \mathfrak{g}_{2}=\bigwedge^{6} V, & \mathfrak{g}_{3}=\bigwedge^{7} V^{*}, \\
\mathfrak{g}_{-1}=\bigwedge^{6} V^{*}, & \mathfrak{g}_{-2}=\bigwedge^{7} V^{*}, & \mathfrak{g}_{-3}=\bigwedge^{7} V .
\end{array}
$$

The algebra $\mathfrak{g}_{\overline{0}}$ is isomorphic to $\mathfrak{o}_{\Pi}^{(2)}(16) \notin \mathbb{K} d$, where $d=E_{6,6}+\cdots+E_{13,13}$, and $\mathfrak{g}_{\overline{1}}$ is an irreducible $\mathfrak{g}_{0}$-module with the highest weight the highest weight element $x_{120}$ of weight $(1,0, \ldots, 0)$ with respect to $h_{1}, \ldots, h_{8} ; \mathfrak{g}_{\overline{1}}$ also possesses a lowest weight vector.

\subsection{Systems of simple roots of the e-type Lie superalgebras}

13.2.1. Remark. Observe that if $p=2$ and the Cartan matrix has no parameters, the reflections do not change the shape of the Dynkin diagram. Therefore, for the $\mathfrak{e}$-superalgebras, it suffices to list distributions of parities of the nodes in order to describe the Dynkin diagrams. Since there are tens and even hundreds of diagrams in these cases, this possibility saves a lot of space, see the lists of all inequivalent Cartan matrices of the $\mathfrak{e}$-type Lie superalgebras. 


\subsection{2 $\mathfrak{e}(6,1) \simeq \mathfrak{e}(6,5)$ of $\operatorname{sdim} 46 \mid 32$}

All inequivalent Cartan matrices are as follows (none of the matrices corresponding to the symmetric pairs of Dynkin diagrams is excluded but are placed one under the other for clarity, followed by three symmetric diagrams):

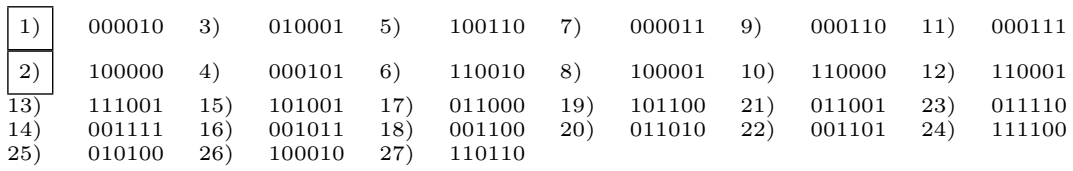

\subsection{3 $\mathfrak{e}(6,6)$ of $\operatorname{sdim}=38 \mid 40$}

All inequivalent Cartan matrices are as follows:

\begin{tabular}{|c|c|c|c|c|c|c|c|c|c|c|c|c|c|}
\hline 1) & 000001 & 2) & 000100 & 3) & 001000 & 4) & 010000 & 5) & 011011 & 6) & 101110 & 7) & 111110 \\
\hline 8) & 011100 & 9) & 101111 & 10) & 011101 & 11) & 101010 & 12) & 111101 & 13) & 010110 & 14) & 101011 \\
\hline 15) & 110011 & 16) & 001001 & 17) & 011111 & 18) & 110100 & 19) & 010011 & 20) & 101000 & 21) & 11101 \\
\hline 22$)$ & 001010 & 23) & 100011 & 24) & 110101 & 25) & 001110 & 26) & 111000 & 27) & 010010 & 28) & 100111 \\
\hline 29) & 100100 & 30) & 110111 & 31) & 100101 & 32) & 111010 & 33) & 010101 & 34) & 010111 & $35)$ & 101101 \\
\hline
\end{tabular}

\subsection{4 $\mathfrak{e}(7,1)$ of $\operatorname{sdim}=80 / 78 \mid 54$}

All inequivalent Cartan matrices are as follows:

\begin{tabular}{llllllllllllll}
\hline 1$)$ & 1000000 & $2)$ & 1000010 & $3)$ & 1000110 & $4)$ & 1001100 & $25)$ & 0110000 & $26)$ & 0110010 & $27)$ & 0110110 \\
\cline { 1 - 5 } & 1010001 & $6)$ & 1011001 & $7)$ & 1100000 & $8)$ & 1100010 & $21)$ & 0011010 & $22)$ & 0011110 & $23)$ & 0100001 \\
$9)$ & 1100110 & $10)$ & 1101100 & $11)$ & 1110001 & $12)$ & 1111001 & $17)$ & 0001101 & $18)$ & 0001111 & $19)$ & 0010100 \\
$13)$ & 0000011 & $14)$ & 0000101 & $15)$ & 0000111 & $16)$ & 0001011 & $28)$ & 0111100 & $24)$ & 0101001 & $20)$ & 0011000
\end{tabular}

\subsection{5 $\mathfrak{e}(7,6)$ of $\operatorname{sdim}=70 / 68 \mid 64$}

All inequivalent Cartan matrices are as follows:

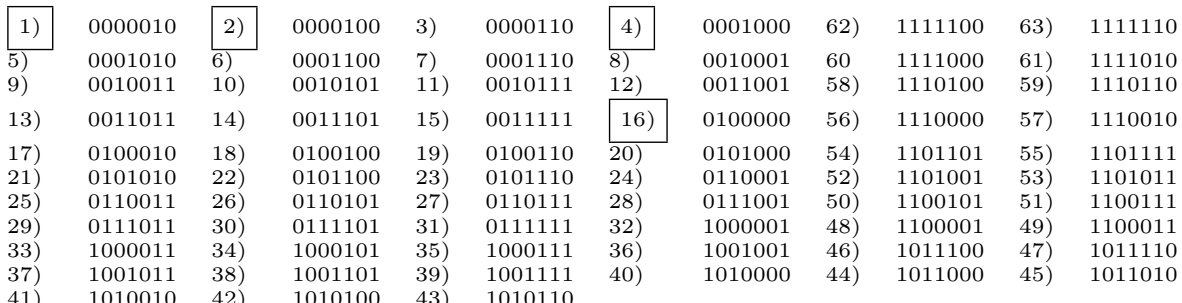

\subsection{6 $\mathfrak{e}(7,7)$ of $\operatorname{sdim}=64 / 62 \mid 70$}

All inequivalent Cartan matrices are as follows:

\begin{tabular}{|c|c|c|c|c|c|c|c|c|c|c|c|}
\hline 1) & 0000001 & 2) & 0001001 & 3) & 0010000 & 4) & 0010010 & 34) & 1111011 & 35) & 1111101 \\
\hline 5) & 0010110 & 6) & 0011100 & 7) & 0100011 & 8) & 0100101 & $32)$ & 1110101 & 33) & 1110111 \\
\hline 9) & 0100111 & 10) & 0101011 & 11) & 0101101 & 12) & 0101111 & 30) & 1101110 & 31) & 1110011 \\
\hline 13) & 0110100 & 14) & 0111000 & 15) & 0111010 & 16) & 0111110 & 28) & 1101000 & 29) & 1101010 \\
\hline 17) & 1000100 & 18) & 1001000 & 19) & 1001010 & 20) & 100 & 26) & 1011111 & 27) & 1100100 \\
\hline 21) & 1010011 & 22) & 1010101 & 23) & 1010111 & 24) & 1011011 & 25) & 1011101 & & \\
\hline
\end{tabular}




\subsection{7 $\mathfrak{e}(8,1)$ of $\operatorname{sdim}=136 \mid 112$}

All inequivalent Cartan matrices are as follows:

\begin{tabular}{llllllllll}
\hline 1$)$ & 10000000 & $2)$ & 10000010 & $3)$ & 10000011 & $4)$ & 10000101 & $120)$ & 01111110 \\
\hline 5$)$ & 10000110 & $6)$ & 10000111 & $7)$ & 10001011 & $8)$ & 10001100 & $119)$ & 01111010 \\
$9)$ & 10001101 & $10)$ & 10001111 & $11)$ & 10010001 & $12)$ & 10010100 & $118)$ & 01111001 \\
$13)$ & 10011000 & $14)$ & 10011001 & $15)$ & 10011010 & $16)$ & 10011110 & $117)$ & 01111000 \\
$17)$ & 10100000 & $18)$ & 10100001 & $19)$ & 10100010 & $20)$ & 10100110 & $116)$ & 01110100 \\
$21)$ & 10101001 & $22)$ & 10101100 & $23)$ & 10110000 & $24)$ & 10110001 & $115)$ & 01110001 \\
$25)$ & 10110010 & $26)$ & 10110110 & 27 & 10111001 & $28)$ & 10111100 & $114)$ & 01101111 \\
$29)$ & 11000000 & $30)$ & 11000010 & $31)$ & 11000011 & $32)$ & 11000101 & $113)$ & 01101101 \\
$33)$ & 11000110 & $34)$ & 11000111 & $35)$ & 11001011 & $36)$ & 11001100 & $112)$ & 01101100 \\
$37)$ & 11001101 & $38)$ & 11001111 & $39)$ & 11010001 & $40)$ & 11010100 & $111)$ & 01101011 \\
$41)$ & 11011000 & $42)$ & 11011001 & $43)$ & 11011010 & $44)$ & 11011110 & $110)$ & 01100111 \\
$45)$ & 11100000 & $46)$ & 11100001 & $47)$ & 11100010 & $48)$ & 11100110 & $109)$ & 01100110 \\
$49)$ & 11101001 & $50)$ & 11101100 & $51)$ & 11110000 & $52)$ & 11110001 & $108)$ & 01100101 \\
$53)$ & 11110010 & $54)$ & 11110110 & $55)$ & 11111001 & $56)$ & 11111100 & $107)$ & 01100011 \\
$57)$ & 00000011 & $58)$ & 00000100 & $59)$ & 00000101 & $60)$ & 00000111 & $106)$ & 01100010 \\
\hline 61$)$ & 00001000 & $62)$ & 00001010 & $63)$ & 00001011 & $64)$ & 00001101 & $105)$ & 01100000 \\
\hline 65$)$ & 00001110 & $66)$ & 00001111 & $67)$ & 00010011 & $68)$ & 00010100 & $104)$ & 01011100 \\
$69)$ & 00010101 & $70)$ & 00010111 & $71)$ & 00011000 & $72)$ & 00011010 & $103)$ & 01011001 \\
$73)$ & 00011011 & $74)$ & 00011101 & $75)$ & 00011110 & $76)$ & 00011111 & $102)$ & 01010110 \\
$77)$ & 00100001 & $78)$ & 00100100 & $79)$ & 00101000 & $80)$ & 00101001 & $101)$ & 01010010 \\
$81)$ & 00101010 & $82)$ & 00101110 & $83)$ & 00110000 & $84)$ & 00110010 & $100)$ & 01010001 \\
$85)$ & 00110011 & $86)$ & 00110101 & $87)$ & 00110110 & $88)$ & 00110111 & $99)$ & 01010000 \\
$89)$ & 00111011 & $90)$ & 00111100 & $91)$ & 00111101 & $92)$ & 00111111 & $98)$ & 01001100 \\
\hline 93$)$ & 01000000 & $94)$ & 01000001 & $95)$ & 01000010 & $96)$ & 01000110 & $97)$ & 01001001
\end{tabular}

\subsection{8 $\mathfrak{e}(8,8)$ of $\operatorname{sdim}=120 \mid 128$}

\section{All inequivalent Cartan matrices are as follows:}

\begin{tabular}{|c|c|c|c|c|c|c|c|c|c|}
\hline 1) & 00000001 & 2) & 00000010 & 12) & 00100000 & 6) & 00010000 & 109) & 11010101 \\
\hline 5$)$ & 00001100 & 4) & 00001001 & 7) & 00010001 & $8)$ & 00010010 & 110) & 11010110 \\
\hline 9) & 00010110 & 10) & 00011001 & 11) & 00011100 & 3) & 00000110 & 111) & 11010111 \\
\hline 13) & 00100010 & 14) & 00100011 & 15) & 00100101 & 16) & 00100110 & 112) & 11011011 \\
\hline 17) & 00100111 & 18) & 00101011 & 19) & 00101100 & 20) & 00101101 & 113) & 11011100 \\
\hline 21) & 00101111 & 22) & 00110001 & 23) & 00110100 & 24) & 00111000 & 114) & 11011101 \\
\hline 25) & 00111001 & 26) & 00111010 & 27) & 00111110 & 28) & 01000011 & 115) & 11011111 \\
\hline 29) & 01000100 & 30) & 01000101 & 31) & 01000111 & 32) & 01001000 & 116) & 11100011 \\
\hline 33) & 01001010 & 34) & 01001011 & $35)$ & 01001101 & 36) & 01001110 & 117) & 11100100 \\
\hline 37) & 01001111 & 38) & 01010011 & 39) & 01010100 & 40) & 01010101 & 118) & 11100101 \\
\hline 41) & 01010111 & 42) & 01011000 & 43) & 01011010 & 44) & 01011011 & 119) & 11100111 \\
\hline 45) & 01011101 & 46) & 01011110 & 47) & 01011111 & 48) & 01100001 & 120) & 11101000 \\
\hline 49) & 01100100 & 50) & 01101000 & 51) & 01101001 & 52) & 01101010 & 121) & 11101010 \\
\hline 53) & 01101110 & 54) & 01110000 & $55)$ & 01110010 & 56) & 01110011 & 122) & 11101011 \\
\hline 57) & 01110101 & 58) & 01110110 & 59) & 01110111 & 60) & 01111011 & 123) & 11101101 \\
\hline 61) & 01111100 & 62) & 01111101 & 63) & 01111111 & 64) & 10000001 & 124) & 11101110 \\
\hline 65$)$ & 10000100 & $66)$ & 10001000 & 67) & 10001001 & 68) & 10001010 & 125) & 11101111 \\
\hline 69) & 10001110 & 70) & 10010000 & 71) & 10010010 & 72) & 10010011 & 126) & 11110011 \\
\hline 73) & 10010101 & 74) & 10010110 & 75) & 10010111 & 76) & 10011011 & 127) & 11110100 \\
\hline 77) & 10011100 & 78) & 10011101 & 79) & 10011111 & 80) & 10100011 & 128) & 11110101 \\
\hline 81) & 10100100 & $82)$ & 10100101 & $83)$ & 10100111 & 84) & 10101000 & 129) & 11110111 \\
\hline 85$)$ & 10101010 & $86)$ & 10101011 & 87) & 10101101 & 88) & 10101110 & 130) & 11111000 \\
\hline 89) & 10101111 & 90) & 10110011 & 91) & 10110100 & 92) & 10110101 & 131) & 11111010 \\
\hline 93) & 10110111 & 94) & 10111000 & $95)$ & 10111010 & 96) & 10111011 & 132) & 11111011 \\
\hline 97) & 10111101 & 98) & 10111110 & 99) & 10111111 & 100) & 11000001 & 133) & 11111101 \\
\hline 101) & 11000100 & 102) & 11001000 & 103) & 11001001 & 104) & 11001010 & 134) & 11111110 \\
\hline & 11001110 & 106) & 11010000 & 107) & 11010010 & 108) & 11010011 & 135) & 1111 \\
\hline
\end{tabular}


14 Table. Dynkin diagrams for $p=2$

\begin{tabular}{|c|c|c|c|c|c|c|}
\hline Diagrams & $\mathfrak{g}$ & $v$ & $e v$ & $o d$ & $p n g$ & $n g \leq \min (*, *)$ \\
\hline \multirow[t]{2}{*}{ 1) } & \multirow{2}{*}{$\begin{array}{l}\mathfrak{o o c}\left(2 ; 2 k_{\overline{0}} \mid 2 k_{\overline{1}}\right) \notin \mathbb{K} I_{0} \\
\text { if } k_{\overline{0}}+k_{\overline{1}} \text { is odd; } \\
\mathfrak{o o c}\left(1 ; 2 k_{\overline{0}} \mid 2 k_{\overline{1}}\right) \notin \mathbb{K} I_{0} \\
\text { if } k_{\overline{0}}+k_{\overline{1}} \text { is even. }\end{array}$} & \multirow[t]{2}{*}{$k_{\overline{0}}+k_{\overline{1}}$} & $\begin{array}{l}k_{\overline{0}}-2 \\
k_{\overline{1}} \\
k_{\overline{1}}-2 \\
k_{\overline{0}}\end{array}$ & $\begin{array}{l}k_{\overline{1}} \\
k_{\overline{0}}-2 \\
k_{\overline{0}} \\
k_{\overline{1}}-2\end{array}$ & $\begin{array}{l}\overline{0} \\
\overline{1} \\
\overline{0} \\
\overline{1}\end{array}$ & $\begin{array}{l}2 k_{\overline{0}}-4,2 k_{\overline{1}} \\
2 k_{\overline{0}}-3,2 k_{\overline{1}}-1 \\
2 k_{\overline{0}}, 2 k_{\overline{1}}-4 \\
2 k_{\overline{0}}-1,2 k_{\overline{1}}-3\end{array}$ \\
\hline & & & $\begin{array}{l}k_{\overline{0}}-1 \\
k_{\overline{1}}-1\end{array}$ & $\begin{array}{l}k_{\overline{1}}-1 \\
k_{\overline{0}}-1\end{array}$ & & $\begin{array}{l}2 k_{\overline{0}}-2,2 k_{\overline{1}}-1 \\
2 k_{\overline{0}}-1,2 k_{\overline{1}}-2 \\
\end{array}$ \\
\hline \multirow{2}{*}{$\begin{array}{l}\text { 3) } \bullet \cdots \cdots \bullet \\
4) \bullet-\cdots \cdots\end{array}$} & \multirow{2}{*}{$\mathfrak{o o}_{\mathrm{II}}^{(1)}\left(2 k_{\overline{0}}+1 \mid 2 k_{\overline{1}}\right)$} & \multirow{2}{*}{$k_{\overline{0}}+k_{\overline{1}}$} & $\begin{array}{l}k_{\overline{0}}-1 \\
k_{\overline{1}}\end{array}$ & $\begin{array}{l}k_{\overline{1}} \\
k_{\overline{0}}-1\end{array}$ & $\begin{array}{l}\overline{0} \\
\overline{1} \\
\end{array}$ & $\begin{array}{l}2 k_{\overline{0}}-2,2 k_{\overline{1}} \\
2 k_{\overline{0}}-1,2 k_{\overline{1}}-1\end{array}$ \\
\hline & & & $\begin{array}{l}k_{\overline{1}}-1 \\
k_{\overline{0}}\end{array}$ & $\begin{array}{l}k_{\overline{0}} \\
k_{\overline{1}}-1\end{array}$ & $\begin{array}{l}\overline{0} \\
\overline{1} \\
\end{array}$ & $\begin{array}{l}2 k_{\overline{0}}, 2 k_{\overline{1}}-2 \\
2 k_{\overline{0}}-1,2 k_{\overline{1}}-1\end{array}$ \\
\hline 5) & $\begin{array}{l}\mathfrak{p e c}(2 ; m) \notin \mathbb{K} I_{0} \\
\text { if } m \text { is odd; } \\
\mathfrak{p e c}(1 ; m) \notin \mathbb{K} I_{0} \\
\text { if } m \text { is even. }\end{array}$ & $m$ & & & & \\
\hline
\end{tabular}

\subsection{Notation}

The Dynkin diagrams in Table 14 correspond to CM Lie superalgebras close to ortho-orthogonal and periplectic Lie superalgebras. Each thin black dot may be $\otimes$ or $\odot$; the last five columns show conditions on the diagrams; in the last four columns, it suffices to satisfy conditions in any one row. Horizontal lines in the last four columns separate the cases corresponding to different Dynkin diagrams. The notations are:

$v$ is the total number of nodes in the diagram;

$n g$ is the number of "grey" nodes $\otimes$ 's among the thin black dots;

$p n g$ is the parity of this number;

$e v$ and $o d$ are the number of thin black dots such that the number of $\otimes$ 's to the left from them is even and odd, respectively. 


\section{Fixed points of symmetries of the Dynkin diagrams}

\subsection{Recapitulation}

For $p=0$, it is well known that the Lie algebras of series $B$ and $C$ and the exceptions $F$ and $G$ are obtained as the sets of fixed points of the outer automorphism of an appropriate Lie algebra of $A D E$ series. All these automorphisms correspond to the symmetries of the respective Dynkin diagram. Not all simple finite dimensional Lie superalgebras can be obtained as the sets of fixed points of the symmetry of an appropriate Dynkin diagram, but many of them can, see [21].

Recall Serganova's result [47] on outer automorphisms (i.e., the modulo the connected component of the unity of the automorphism group) of simple finite dimensional Lie superalgebras for $p=0$. The symmetry of the Dynkin diagram of $\mathfrak{s l}(n)$ corresponds to the transposition with respect to the side diagonal, conjugate in the group of automorphisms of $\mathfrak{s l}(n)$ to the "minus transposition" $X \mapsto-X^{t}$. In the super case, this automorphism becomes $X \longmapsto-X^{s t}$, where

$$
\left(\begin{array}{ll}
A & B \\
C & D
\end{array}\right)^{s t}=\left(\begin{array}{cc}
A^{t} & -C^{t} \\
B^{t} & D^{t}
\end{array}\right) .
$$

This automorphism, seemingly of order 4 , is actually of order 2 modulo the connected component of the unity of the automorphism group, and is of order 4 only for $\mathfrak{s l}(2 n+1 \mid 2 m+1)$.

The queer Lie superalgebra $\mathfrak{q}(n)$ is obtained as the set of fixed points of the automorphism

$$
\Pi:\left(\begin{array}{cc}
A & B \\
C & D
\end{array}\right) \longmapsto\left(\begin{array}{cc}
D & C \\
B & A
\end{array}\right)
$$

of $\mathfrak{g l}(n \mid n)$ corresponding to the symmetry of the Dynkin diagram

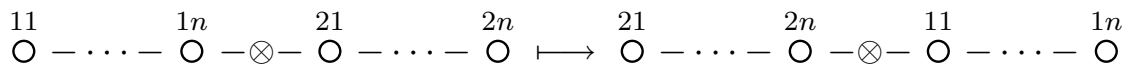

which interchanges the identical maximal parts $\mathrm{O}_{-} \cdot \cdots-\mathrm{O}$ preserving the order of nodes; whereas $\mathfrak{p} \mathfrak{e}(n)$ is the set of fixed points of the composition automorphism $\Pi \circ(-s t)$.

\subsection{New result}

The modular version of the above statements is given in the next Theorem in which, speaking about ortho-orthogonal and periplectic superalgebras, we distinguish the cases where the fork node is grey or white $(g \mathfrak{g}(A)$ and $w \mathfrak{g}(A)$, respectively); to squeeze the data in the table, we write $\widehat{\mathfrak{g}}$ instead of $\mathfrak{g} \in \mathbb{K} I_{0}$. We also need the following decomposable Cartan matrices $(p=2)$ :

$$
\mathcal{N}:=\left(\begin{array}{cccc}
\overline{0} & 1 & 0 & 0 \\
1 & \overline{0} & 0 & 0 \\
0 & 1 & \overline{0} & 1 \\
0 & 0 & 1 & \overline{0}
\end{array}\right), \quad \mathcal{M}:=\left(\begin{array}{cccc}
\overline{0} & 1 & 0 & 0 \\
1 & 0 & 0 & 0 \\
0 & 1 & \overline{0} & 1 \\
0 & 0 & 1 & \overline{0}
\end{array}\right)
$$

\subsubsection{The Lie algebra $\mathfrak{g}(\mathcal{N})$}

It is of $\operatorname{dim} 34$ and not simple; it contains a simple ideal of $\operatorname{dim}=26$ which is $\mathfrak{o}(1 ; 8)^{(1)} / \mathfrak{c}$ and the quotient is isomorphic to $\mathfrak{s l}(3)$.

\subsubsection{The Lie superalgebra $\mathfrak{g}(\mathcal{M})$}

It is of $\operatorname{sdim} 18 \mid 16$ and not simple. Its even part is $\mathfrak{h} \mathfrak{e i}(2) \oplus_{c} \mathfrak{g}(C)$, where $\mathfrak{h} \mathfrak{e i}(2)=\operatorname{Span}\left\{X^{ \pm}, c\right\}$ and $c$ is the center of the Lie algebra $\mathfrak{g}(C)$, where

$$
C:=\left(\begin{array}{ccc}
\overline{0} & 0 & 0 \\
0 & \overline{0} & 1 \\
0 & 1 & \overline{0}
\end{array}\right) \text {. }
$$


The brackets are as follows:

$$
\left[X^{ \pm}, \mathfrak{g}(C)^{(1)}\right]=0, \quad\left[X^{ \pm}, d\right]=X^{ \pm}, \quad\left[X^{+}, X^{-}\right]=c
$$

where $d$ is the grading operator of the Lie algebra $\mathfrak{g}(C)$.

Now the Cartan subalgebra of $\mathfrak{g}(\mathcal{M})$ is generated by $h_{3}, h_{6}, h_{1}+h_{5}, h_{2}+h_{4}$ and the highest weight vector of the module $\mathfrak{g}(\mathcal{M})_{\overline{1}}$ is $x_{32}+x_{33}$, where

$$
x_{32}=\left[\left[\left[x_{1}, x_{2}\right],\left[x_{3}, x_{4}\right]\right],\left[\left[x_{3}, x_{6}\right],\left[x_{4}, x_{5}\right]\right]\right], \quad x_{33}=\left[\left[\left[x_{1}, x_{2}\right],\left[x_{3}, x_{6}\right]\right],\left[\left[x_{2}, x_{3}\right],\left[x_{4}, x_{5}\right]\right]\right] .
$$

Its weight is $(0,0,1,0)$ (according to the ordering of the generators of the Cartan subalgebra as above).

The restriction of the module to $\mathfrak{h e i}(2)$ consists of 8 copies of the 2-dimensional irreducible Fock module; the restriction to $\mathfrak{g}(C)$ consists of 2 copies of an irreducible 8-dimensional module.

The lowest weight vector is $y_{32}+y_{33}$ with weight $(0,0,1,0)$.

The Lie superalgebra $\mathfrak{g}(\mathcal{M})$ has a simple ideal, of sdim $=10 \mid 16$ which is $\mathfrak{o o}(1 ; 4 \mid 4)^{(1)} / \mathfrak{c}$ (to be described separately below) and the quotient is isomorphic to $\mathfrak{s l}(3)$.

15.2.3. Theorem. If the Dynkin diagram of $i \mathfrak{g}(A)$ is symmetric, it gives rise to an outer automorphism $\sigma$ whose fixed points constitute the Lie superalgebra $(i \mathfrak{g}(A))^{\sigma}$ which occupies the slot under $i \mathfrak{g}(A)$ in the following tables (15.1), (15.2), (15.3).

1) $p=2$ : The order 2 automorphisms of the $\mathfrak{s l}$ series corresponding to the symmetries of Dynkin diagrams give the following fixed points, where $\sigma=t$ is the transposition, unless otherwise

\begin{tabular}{|c|c|c|c|}
\hline $\mathfrak{s l}(2 n+1)$ & $\mathfrak{g l}(2 n)$ & $\mathfrak{s l}(2 k+1 \mid 2 m)$ & \\
\hline $\mathfrak{o}(2 n+1)$ & $\mathfrak{o}(2 n)$ & $\mathfrak{o} \mathfrak{o}(2 k+1 \mid 2 m)$ & $\mathfrak{g l}(2 k+1 \mid 2 m+1)$ \\
\hline $\mathfrak{g l}(n \mid n)$ & $\mathfrak{g l}(n \mid n)$ & $\mathfrak{g l}(2 k \mid 2 m)$ & $\mathfrak{o} \mathfrak{o}(2 k+1 \mid 2 m+1)$ \\
\hline $\mathfrak{q}(n), \quad \sigma=\Pi$ & $\mathfrak{p} \mathfrak{e}(n), \quad \sigma=\Pi \circ(t)$ & $\mathfrak{o} \mathfrak{o}(2 k \mid 2 m)$ & \\
\hline
\end{tabular}
stated:

2) $p=2:$ The order 2 automorphisms of the orthogonal and ortho-orthogonal series give the following fixed points (recall the definition of $\hat{\mathfrak{g}}$ in (6.12)):

\begin{tabular}{|ll|ll|}
$\operatorname{ooc}\left(\widehat{2 ; 2 k_{\overline{0}}} \mid 2 k_{\overline{1}}\right)$ & for $k_{\overline{0}}+k_{\overline{1}}$ odd & $\widehat{\mathfrak{o c}(2 ; 2 k)}$ & for $k$ odd \\
$\operatorname{ooc}\left(\widehat{1 ; 2 k_{\overline{0}}} \mid 2 k_{\overline{1}}\right)$ & for $k_{\overline{0}}+k_{\overline{1}}$ even & $\widehat{\mathfrak{o c}(1 ; 2 k)}$ & for $k$ even \\
\hline
\end{tabular}

3) The following are the fixed points of order 2 automorphisms of the exceptional Lie (super)algebras for $p=3$, and also, for $p=2$, of the periplectic superalgebras, and of order 3

\begin{tabular}{|c|c|c|c|c|c|}
\hline $\begin{array}{l}1 \mathfrak{g}(2,3) \\
\mathfrak{p} \mathfrak{s l}(2 \mid 2)\end{array}$ & $\begin{array}{l}2 \mathfrak{g}(2,3) \\
\mathfrak{s l}(1 \mid 2)\end{array}$ & $\begin{array}{l}5 \mathfrak{g}(2,3) \\
\mathfrak{o s p}(3 \mid 2)\end{array}$ & $\begin{array}{l}5 \mathfrak{g}(2,6) \\
\mathfrak{g}(1,6)\end{array}$ & $\begin{array}{l}2 \mathfrak{g}(2,6) \\
\mathfrak{g}(1,6)\end{array}$ & $\begin{array}{l}\widehat{\mathfrak{o c}(1 ; 8)} \\
\mathfrak{g l}(4)\end{array}$ \\
\hline$g \mathfrak{o o \mathfrak { c } ( 1 ; 4 | 4 )}$ & $w \mathfrak{o o c}(1 ; 4 \mid 4)$ & $\widehat{g \mathfrak{p} \mathfrak{e} \mathfrak{c}(1 ; 4)}$ & $\widehat{w \mathfrak{p e c}(1 ; 4)}$ & $g \mathfrak{o o \mathfrak { c } ( 2 ; 6} \mid 2)$ & $w \mathfrak{o o \mathfrak { c } ( 2 ; 6} \mid 2)$ \\
\hline $\mathfrak{g l}(2 \mid 2)$ & $\mathfrak{g l}(2 \mid 2)$ & $\mathfrak{g l}(1 \mid 3)$ & $\mathfrak{g l}(1 \mid 3)$ & $\mathfrak{g l}(2 \mid 2)$ & $\mathfrak{g l}(2 \mid 2)$ \\
\hline
\end{tabular}
automorphisms of the orthogonal algebra and ortho-orthogonal superalgebras.

Besides, $\mathfrak{e}(6)^{\sigma}=\mathfrak{g}(\mathcal{N})$, whereas

$$
\begin{aligned}
& 25 \mathfrak{e}(6,1)^{\sigma} \simeq 26 \mathfrak{e}(6,1)^{\sigma} \simeq 27 \mathfrak{e}(6,1)^{\sigma} \simeq \\
& 1 \mathfrak{e}(6,6)^{\sigma} \simeq 7 \mathfrak{e}(6,6)^{\sigma} \simeq 5 \mathfrak{e}(6,6)^{\sigma} \simeq 33 \mathfrak{e}(6,6)^{\sigma} \simeq 8 \mathfrak{e}(6,6)^{\sigma} \simeq 29 \mathfrak{e}(6,6)^{\sigma} \simeq \\
& 32 \mathfrak{e}(6,6)^{\sigma} \simeq 10 \mathfrak{e}(6,6)^{\sigma} \simeq 14 \mathfrak{e}(6,6)^{\sigma} \simeq 18 \mathfrak{e}(6,6)^{\sigma} \simeq 28 \mathfrak{e}(6,6)^{\sigma} \simeq 36 \mathfrak{e}(6,6)^{\sigma} \simeq \mathfrak{g}(\mathcal{M}) .
\end{aligned}
$$




\section{A realization of $\mathfrak{g}=\mathfrak{o} \mathfrak{o}(4 \mid 4)^{(1)} / \mathfrak{c}$}

This simple Lie superalgebra $\mathfrak{g}$ admits a realization in which $\mathfrak{g}_{\overline{0}} \simeq \mathfrak{h} \mathfrak{e}(8) \notin \mathbb{K} E$, where $\mathfrak{h} \mathfrak{e}(8)=$ $\operatorname{Span}(p, q, c)$ with $p=\left(p_{1}, \ldots, p_{4}\right), q=\left(q_{1}, \ldots, q_{4}\right)$, and $E:=\sum\left(p_{i} \partial_{p_{i}}+q_{i} \partial_{q_{i}}\right)$ and with $c$ being central in $\mathfrak{h} \mathfrak{e} \mathfrak{i}$, and in which $\mathfrak{g}_{\overline{1}}$ is a copy of the Fock space (over $\mathfrak{h} \mathfrak{e}(8)$ ) considered purely odd, i.e., as $\Pi\left(\mathbb{K}[p] /\left(p_{1}^{2}, \ldots, p_{4}^{2}\right)\right)$. (Obviously, the indeterminates $p$ and $q$, as well as $\xi$ and $\eta$, cf. Section 7.1.1, are interchangeable.)

Indeed, consider the following isomorphism

$$
\begin{aligned}
& \varphi: \Pi\left(\mathbb{K}[p] /\left(p_{1}^{2}, \ldots, p_{4}^{2}\right)\right) \longrightarrow \operatorname{Span}\left(\varphi_{0}, \ldots, \varphi_{1234}\right), \\
& \varphi_{0}:=\Pi(1), \varphi_{i}:=\Pi\left(p_{i}\right), \varphi_{i j}:=\Pi\left(p_{i} p_{j}\right), \ldots, \varphi_{1234}:=\Pi\left(p_{1} p_{2} p_{3} p_{4}\right) .
\end{aligned}
$$

\begin{tabular}{|c|c|c|c|c|c|c|c|c|c|c|c|c|c|c|c|c|}
\hline & $\varphi_{1234}$ & $\varphi_{234}$ & $\varphi_{134}$ & $\varphi_{124}$ & $\varphi_{123}$ & $\varphi_{34}$ & $\varphi_{24}$ & $\varphi_{23}$ & $\varphi_{14}$ & $\varphi_{13}$ & $\varphi_{12}$ & $\varphi_{4}$ & $\varphi_{3}$ & $\varphi_{2}$ & $\varphi_{1}$ & $\varphi_{0}$ \\
\hline$\varphi_{1234}$ & 0 & 0 & 0 & 0 & 0 & 0 & 0 & 0 & 0 & 0 & 0 & $p_{4}$ & $p_{3}$ & $p_{2}$ & $p_{1}$ & $D$ \\
\hline$\varphi_{234}$ & 0 & 0 & 0 & 0 & 0 & 0 & 0 & 0 & $p_{4}$ & $p_{3}$ & $p_{2}$ & 0 & 0 & 0 & $E$ & $q_{1}$ \\
\hline$\varphi_{134}$ & 0 & 0 & 0 & 0 & 0 & 0 & $p_{4}$ & $p_{3}$ & 0 & 0 & $p_{1}$ & 0 & 0 & $E$ & 0 & $q_{2}$ \\
\hline$\varphi_{124}$ & 0 & 0 & 0 & 0 & 0 & $p_{4}$ & 0 & $p_{2}$ & 0 & $p_{1}$ & 0 & 0 & $E$ & 0 & 0 & $q_{3}$ \\
\hline$\varphi_{123}$ & 0 & 0 & 0 & 0 & 0 & $p_{3}$ & $p_{2}$ & 0 & $p_{1}$ & 0 & 0 & $E$ & 0 & 0 & 0 & $q_{4}$ \\
\hline$\varphi_{34}$ & 0 & 0 & 0 & $p_{4}$ & $p_{3}$ & 0 & 0 & 0 & 0 & 0 & $D$ & 0 & 0 & $q_{1}$ & $q_{2}$ & 0 \\
\hline$\varphi_{24}$ & 0 & 0 & $p_{4}$ & 0 & $p_{2}$ & 0 & 0 & 0 & 0 & $D$ & 0 & 0 & $q_{1}$ & 0 & $q_{3}$ & 0 \\
\hline$\varphi_{23}$ & 0 & 0 & $p_{3}$ & $p_{2}$ & 0 & 0 & 0 & 0 & $D$ & 0 & 0 & $q_{1}$ & 0 & 0 & $q_{4}$ & 0 \\
\hline$\varphi_{14}$ & 0 & $p_{4}$ & 0 & 0 & $p_{1}$ & 0 & 0 & $D$ & 0 & 0 & 0 & 0 & $q_{2}$ & $q_{3}$ & 0 & 0 \\
\hline$\varphi_{13}$ & 0 & $p_{3}$ & 0 & $p_{1}$ & 0 & 0 & $D$ & 0 & 0 & 0 & 0 & $q_{2}$ & 0 & $q_{4}$ & 0 & 0 \\
\hline$\varphi_{12}$ & 0 & $p_{2}$ & $p_{1}$ & 0 & 0 & $D$ & 0 & 0 & 0 & 0 & 0 & $q_{3}$ & $q_{4}$ & 0 & 0 & 0 \\
\hline$\varphi_{4}$ & $p_{4}$ & 0 & 0 & 0 & $E$ & 0 & 0 & $q_{1}$ & 0 & $q_{2}$ & $q_{3}$ & 0 & 0 & 0 & 0 & 0 \\
\hline$\varphi_{3}$ & $p_{3}$ & 0 & 0 & $E$ & 0 & 0 & $q_{1}$ & 0 & $q_{2}$ & 0 & $q_{4}$ & 0 & 0 & 0 & 0 & 0 \\
\hline$\varphi_{2}$ & $p_{2}$ & 0 & $E$ & 0 & 0 & $q_{1}$ & 0 & 0 & $q_{3}$ & $q_{4}$ & 0 & 0 & 0 & 0 & 0 & 0 \\
\hline$\varphi_{1}$ & $p_{1}$ & $E$ & 0 & 0 & 0 & $q_{2}$ & $q_{3}$ & $q_{4}$ & 0 & 0 & 0 & 0 & 0 & 0 & 0 & 0 \\
\hline$\varphi_{0}$ & $D$ & $q_{1}$ & $q_{2}$ & $q_{3}$ & $q_{4}$ & 0 & 0 & 0 & 0 & 0 & 0 & 0 & 0 & 0 & 0 & 0 \\
\hline
\end{tabular}

Now the multiplication is given by the following two tables, where $D:=c+E$ to save space:

\begin{tabular}{|c|cccccccccc|}
\hline & $c$ & $D$ & $p_{1}$ & $p_{2}$ & $p_{3}$ & $p_{4}$ & $q_{1}$ & $q_{2}$ & $q_{3}$ & $q_{4}$ \\
\hline$\varphi_{0}$ & $\varphi_{0}$ & $\varphi_{0}$ & $\varphi_{1}$ & $\varphi_{2}$ & $\varphi_{3}$ & $\varphi_{4}$ & 0 & 0 & 0 & 0 \\
\hline$\varphi_{1}$ & $\varphi_{1}$ & 0 & 0 & $\varphi_{12}$ & $\varphi_{13}$ & $\varphi_{14}$ & $\varphi_{0}$ & 0 & 0 & 0 \\
\hline$\varphi_{2}$ & $\varphi_{2}$ & 0 & $\varphi_{12}$ & 0 & $\varphi_{23}$ & $\varphi_{24}$ & 0 & $\varphi_{0}$ & 0 & 0 \\
\hline$\varphi_{3}$ & $\varphi_{3}$ & 0 & $\varphi_{13}$ & $\varphi_{23}$ & 0 & $\varphi_{34}$ & 0 & 0 & $\varphi_{0}$ & 0 \\
\hline$\varphi_{4}$ & $\varphi_{4}$ & 0 & $\varphi_{14}$ & $\varphi_{24}$ & $\varphi_{34}$ & 0 & 0 & 0 & 0 & $\varphi_{0}$ \\
\hline$\varphi_{12}$ & $\varphi_{12}$ & $\varphi_{12}$ & 0 & 0 & $\varphi_{123}$ & $\varphi_{124}$ & $\varphi_{2}$ & $\varphi_{1}$ & 0 & 0 \\
\hline$\varphi_{13}$ & $\varphi_{13}$ & $\varphi_{13}$ & 0 & $\varphi_{123}$ & 0 & $\varphi_{134}$ & $\varphi_{3}$ & 0 & $\varphi_{1}$ & 0 \\
\hline$\varphi_{14}$ & $\varphi_{14}$ & $\varphi_{14}$ & 0 & $\varphi_{124}$ & $\varphi_{134}$ & 0 & $\varphi_{4}$ & 0 & 0 & $\varphi_{1}$ \\
\hline$\varphi_{23}$ & $\varphi_{23}$ & $\varphi_{23}$ & $\varphi_{123}$ & 0 & 0 & $\varphi_{234}$ & 0 & $\varphi_{3}$ & $\varphi_{2}$ & 0 \\
\hline$\varphi_{24}$ & $\varphi_{24}$ & $\varphi_{24}$ & $\varphi_{124}$ & 0 & $\varphi_{234}$ & 0 & 0 & $\varphi_{4}$ & 0 & $\varphi_{2}$ \\
\hline$\varphi_{34}$ & $\varphi_{34}$ & $\varphi_{34}$ & $\varphi_{134}$ & $\varphi_{234}$ & 0 & 0 & 0 & 0 & $\varphi_{4}$ & $\varphi_{3}$ \\
\hline$\varphi_{123}$ & $\varphi_{123}$ & 0 & 0 & 0 & 0 & $\varphi_{1234}$ & $\varphi_{23}$ & $\varphi_{13}$ & $\varphi_{12}$ & 0 \\
\hline$\varphi_{124}$ & $\varphi_{124}$ & 0 & 0 & 0 & $\varphi_{1234}$ & 0 & $\varphi_{24}$ & $\varphi_{14}$ & 0 & $\varphi_{12}$ \\
\hline$\varphi_{134}$ & $\varphi_{134}$ & 0 & 0 & $\varphi_{1234}$ & 0 & 0 & $\varphi_{34}$ & 0 & $\varphi_{14}$ & $\varphi_{13}$ \\
\hline$\varphi_{234}$ & $\varphi_{234}$ & 0 & $\varphi_{1234}$ & 0 & 0 & 0 & 0 & $\varphi_{34}$ & $\varphi_{24}$ & $\varphi_{23}$ \\
\hline$\varphi_{1234}$ & $\varphi_{1234}$ & $\varphi_{1234}$ & 0 & 0 & 0 & 0 & $\varphi_{234}$ & $\varphi_{134}$ & $\varphi_{124}$ & $\varphi_{123}$ \\
\hline
\end{tabular}

16.1. Remark. If $p=0$, every irreducible module over a solvable Lie algebra is 1-dimensional. A theorem, based on this fact, states that any Lie superalgebra $\mathfrak{g}$ is solvable if and only if $\mathfrak{g}_{\overline{0}}$ is solvable. The example above shows that if $p>0$, life is much more interesting.

We were unable to answer: For $2 n \neq 8$, is there a simple Lie superalgebra $\mathfrak{G}(2 n)$ with $\mathfrak{G}(2 n)_{\overline{0}} \simeq \mathfrak{h e i}(2 n) \in \mathbb{K} E$ and $\mathfrak{G}(2 n)_{\overline{1}} \simeq \Pi($ Fock module over $\mathfrak{h e i}(2 n))$ ? In the next subsection we cite Irina Shchepochkina's answer to this question. 


\subsection{Shchepochkina's comments. Other simple Lie superalgebras with solvable even part}

We consider $\mathfrak{g}=\mathfrak{g}_{0} \oplus \mathfrak{g}_{\overline{1}}$, where

$$
\mathfrak{g}_{\overline{0}}=\mathfrak{h} \mathfrak{e i}(2 n) \notin \mathbb{K} \cdot E=\operatorname{Span}\left(p_{1}, \ldots, p_{n}, q_{1}, \ldots, q_{n}, c, E\right), \quad \text { with } c \text { central, }
$$

i.e.,

$$
\left[p_{i}, q_{j}\right]=\delta_{i j} c, \quad\left[E, p_{i}\right]=p_{i}, \quad\left[E, q_{i}\right]=q_{i},
$$

and $\mathfrak{g}_{\overline{1}}=\Pi\left(\Lambda\left(p_{1}, \ldots, p_{n}\right)\right)$. The Lie algebra $\mathfrak{h e i}(2 n)$ acts in $\mathfrak{g}_{\overline{1}}$ as in the Fock space:

$$
\operatorname{ad}_{c}\left|\mathfrak{g}_{\overline{1}}=\operatorname{id}_{\mathfrak{g}_{\overline{1}}}, \quad \operatorname{ad}_{p_{i}}\right| \mathfrak{g}_{\overline{1}}=p_{i}, \quad \operatorname{ad}_{q_{i}} \mid \mathfrak{g}_{\overline{1}}=\partial_{p_{i}} .
$$

The space $\mathfrak{g}_{\overline{1}}$ is spanned by $\varphi_{0}:=1$, and $\varphi_{i_{1} \ldots i_{k}}:=\Pi\left(p_{i_{1}} \ldots p_{i_{k}}\right)$ for all sets $I$ of distinct indices. For any $I$, let $I^{*}$ denote the complementary set to $\{1, \ldots, n\}$. For clarity, we set $\varphi_{I}^{*}=\varphi_{I^{*}}$.

How can the operator $E$ act in $\mathfrak{g}_{1}$ ? Let us begin with $E \varphi_{0}$ :

$$
\left[q_{i}, E \varphi_{0}\right]=\left[\left[q_{i}, E\right], \varphi_{0}\right]+\left[E,\left[q_{i}, \varphi_{0}\right]\right]=0 \quad \text { for all } i \text {. }
$$

But there is only one (up to a constant factor) element in $\mathfrak{g}_{\overline{1}}$ annihilated by all the $q_{i}$, namely $\varphi_{0}$. Hence $E \varphi_{0}=\lambda \cdot \varphi_{0}$.

Since $\mathfrak{g}_{\overline{1}}$ is generated from $\varphi_{0}$ under the action of operators $p_{i}$ of weight 1 with respect to $E$, the action of $E$ on the monomial $\varphi$ is of the form:

$$
E \varphi=(\lambda+\operatorname{deg} \varphi) \varphi, \quad \text { i.e., } \quad \operatorname{ad}_{E}\left|\mathfrak{g}_{\overline{1}}=\lambda \cdot \operatorname{id}\right| \mathfrak{g}_{\overline{1}}+\operatorname{deg} .
$$

Replacing $E$ by $E+\lambda \cdot c$ (this does not affect the commutation relations in $\mathfrak{g}_{0}$ ), we may assume that $\operatorname{ad}_{E} \mid \mathfrak{g}_{\overline{1}}=$ deg.

Let us try to define the bracket on $\mathfrak{g}_{\overline{1}}$. I claim that it suffices to determine the only bracket (determine $x$ )

$$
x=\left[\varphi_{0}, \varphi_{0}^{*}\right] .
$$

Everything else follows from the Jacobi identity. Indeed,

$$
\left[\varphi_{0}, \varphi_{i}^{*}\right]=\left[\varphi_{0},\left[q_{i}, \varphi_{0}^{*}\right]\right]=\left[\left[\varphi_{0}, q_{i}\right], \varphi_{0}^{*}\right]+\left[q_{i},\left[\varphi_{0}, \varphi_{0}^{*}\right]\right]=\left[q_{i}, x\right],
$$

and the inverse induction on the degree of monomials $\varphi$ yields all the brackets $\left[\varphi_{0}, \varphi\right]$ :

$$
\left[\varphi_{0}, \varphi_{I \cup i}^{*}\right]=\left[\varphi_{0},\left[q_{i}, \varphi_{I}^{*}\right]\right]=\left[\left[\varphi_{0}, q_{i}\right], \varphi_{I}^{*}\right]+\left[q_{i},\left[\varphi_{0}, \varphi_{I}^{*}\right]\right]=\left[q_{i},\left[\varphi_{0}, \varphi_{I}^{*}\right]\right] .
$$

If we know the brackets of a monomial $\psi$ with all monomials of the form $\varphi$, we can recover the brackets of the form $\left[p_{i} \cdot \psi, \varphi\right]$ :

$$
\left[p_{i} \cdot \psi, \varphi\right]=\left[\left[p_{i}, \psi\right], \varphi\right]=\left[p_{i},[\psi, \varphi]\right]+\left[\psi,\left[p_{i}, \varphi\right]\right] .
$$

Thus, by the induction on the degree of $\psi$ we recover all the brackets from the brackets of the form $\left[\varphi_{0}, \varphi\right]$. Equations (16.4)-(16.5) imply that $\left[\mathfrak{g}_{\overline{1}}, \mathfrak{g}_{\overline{1}}\right] \subset \mathfrak{g}_{\overline{0}}^{\prime}+\mathbb{K} \cdot x$, where $\mathfrak{g}_{0}^{\prime}:=\left[\mathfrak{g}_{\overline{0}}, \mathfrak{g}_{\overline{0}}\right]$.

For $\mathfrak{g}$ to be simple, we should have $\left[\mathfrak{g}_{\overline{1}}, \mathfrak{g}_{\overline{1}}\right]=\mathfrak{g}_{\overline{0}}$, i.e., $x \notin \mathfrak{g}_{\overline{0}}^{\prime}$. But $\varphi_{0}$ and $\varphi_{0}^{*}$ are eigenvectors of the operator $E$ of weight 0 and $n$, respectively. Hence their bracket, $x$, is an eigenvector of weight $n$ and, since $x$ should have a non-zero projection to $E$, the number $n$ must be even:

$$
x=\alpha \cdot E+\beta \cdot c, \quad \text { where } \alpha \neq 0 .
$$


Note that we have one more degree of freedom: We can multiply all elements of $\mathfrak{g}_{\overline{1}}$ by the same non-zero scalar. This helps us to fix $\alpha=1$. Thus, $x=E+\beta \cdot c$.

Now, using equations (16.4)-(16.5) we can recover the general formula for the bracket in $\mathfrak{g}_{1}$. I claim that it is of the following beautiful form

$$
[f, g]=c \int\left(\operatorname{ad}_{E}(f) g\right)+(E+\beta c) \int\left(\operatorname{ad}_{c}(f) g\right)+\sum\left(p_{i} \int\left(\operatorname{ad}_{q_{i}}(f) g\right)+q_{i} \int\left(\operatorname{ad}_{p_{i}}(f) g\right),\right.
$$

where $\int$ is the Berezin integral $=$ the coefficient of the highest term (once the basis of the Grassmann algebra is chosen). To see this, it suffices to verify that (16.6) is invariant with respect to $\operatorname{ad}_{q_{i}}$ and $\operatorname{ad}_{p_{i}}$ (the invariance with respect to $\operatorname{ad}_{c}$ and $\operatorname{ad}_{E}$ is obvious).

Here comes an incomplete argument. It remains to verify the Jacobi identity only for triples of odd elements, moreover, it suffices to check it only for triples of the form $\varphi_{0}, \varphi_{0}^{*}, \varphi$. We have

$$
\left[\left[\varphi_{0}, \varphi_{0}^{*}\right], \varphi\right]=(E+\beta \cdot c) \varphi=(\operatorname{deg} \varphi+\beta) \varphi .
$$

What can one say about the sum

$$
\left[\left[\varphi_{0}, \varphi\right], \varphi_{0}^{*}\right]+\left[\varphi_{0},\left[\varphi_{0}^{*}, \varphi\right]\right] ?
$$

Observe that the first summand can only be non-zero if $\varphi=\varphi_{0}^{*}$ or $\varphi_{i}^{*}$, the second summand can only be non-zero if $\varphi=\varphi_{0}$ or $\varphi_{i}$.

For $\varphi=\varphi_{0}^{*}$ and $\varphi=\varphi_{0}$, the Jacobi identity holds for any $\beta$, whereas for $\varphi=\varphi_{i}^{*}$ and $\varphi=\varphi_{i}$ only if $\beta=1$ for $n=2$ and $\beta=0$ for $n>2$.

For $n=2$, the above-listed possibilities exhaust all possible values of $\varphi$.

For $n=4$, there are also elements $\varphi=\varphi_{i j}$ yielding 0 in both formulas. However, for $n>4$ and $\varphi=\varphi_{123}$, equation (16.7) yields $\varphi_{123}$, whereas equation (16.8) yields 0 , so there is no Lie superalgebra.

Thus, for $n=2$, one may have a Lie superalgebra $\mathfrak{G}(4)$ with brackets of its odd elements (the squares of each odd element being 0 )

$$
\left[\varphi_{1}, \varphi_{2}\right]=E, \quad\left[\varphi_{0}, \varphi_{12}\right]=E+c,
$$

where in the right hand sides stand the elements that act on the odd part of the hypothetical Lie superalgebra $\mathfrak{G}(4)$ as in (16.3), i.e., $E$ counts the degree of the element of the Grassmann algebra; the other bracket being defined similar to (16.2):

$$
\left[\varphi_{i}, \varphi_{12}\right]=p_{i}, \quad\left[\varphi_{0}, \varphi_{1}\right]=q_{2}, \quad\left[\varphi_{0}, \varphi_{2}\right]=q_{1},
$$

the element $p_{i}$ acts on $\mathfrak{G}(4)_{\overline{1}}$ as the multiplication by $p_{i}$ (i.e., $\left[p_{i}, \varphi_{0}\right]=\varphi_{i},\left[p_{i}, \varphi_{j}\right]=\varphi_{i j}$ and so on), and $q_{i}$ as $\partial_{p_{i}}$.

Here comes the complete argument: In the above argument we forgot that the Jacobi identity for $p=2$ and odd elements is of the form (3.3), not of the usual form $[x,[x, x]]=0$. And taking $x=\varphi_{0}, y=p_{1}$ we fail to satisfy the Jacobi identity although it is so tempting to set

$$
x_{1}:=q_{1}, \quad x_{2}:=\varphi_{1}, \quad y_{1}:=p_{1}, \quad y_{2}:=\varphi_{2}
$$

and (correctly) deduce that the relations between these $x$ 's and $y$ 's yield the same Cartan matrix of $\mathfrak{G}(4)$ as that of $\mathfrak{o o}_{\mathrm{II}}^{(1)}(1 \mid 4)$. However, due to squaring the space of $\mathfrak{G}(4)$ is not a Lie superalgebra.

There are, however, simple Lie superalgebras with solvable even part other than $\mathfrak{o o}_{\Pi \Pi}^{(1)}(4 \mid 4) / \mathfrak{c}$. Indeed, we know that the Lie algebras $\mathfrak{o}_{\mathrm{I}}^{(1)}(n)$ are solvable (only) for $n=1$ and 2 , and $\mathfrak{o}_{\Pi}(n)$ are solvable (only) for $n=2$ and 4 . Therefore the Lie superalgebras

$$
\begin{aligned}
& \mathfrak{o o}_{\mathrm{II}}^{(1)}(1 \mid 2), \quad \mathfrak{o o}_{\mathrm{II}}^{(1)}(1 \mid 2), \quad \mathfrak{o o}_{\mathrm{II}}^{(1)}(2 \mid 2), \quad \mathfrak{o o}_{\mathrm{II}}^{(1)}(2 \mid 2), \\
& \mathfrak{o o}_{\Pi \Pi}^{(1)}(2 \mid 4), \quad \mathfrak{o o}_{\Pi \Pi}^{(2)}(2 \mid 4), \quad \mathfrak{o o}_{\Pi \Pi}^{(1)}(4 \mid 4),
\end{aligned}
$$


though simple (perhaps, modulo center), have solvable even parts. One can not get a simple Lie superalgebra from $\mathfrak{o o}_{\Pi \Pi}^{(1)}(2 \mid 2)$ passing to derived and factorizing.

\section{Acknowledgements}

We are very thankful to A. Lebedev for help (he not only clarified the notion of $\mathfrak{g}(A)$ and roots, but also helped us to figure out the structure of $\mathfrak{g}(A)$ in the most complicated cases and elucidate the notion of $p$-structure, he also listed inequivalent Cartan matrices for the $\mathfrak{e}$-cases) and to I. Shchepochkina for her contribution. We thank A. Protopopov for his help with our graphics, see [43]. Constructive criticism of referees is most thankfully acknowledged; the paper is much clearer now.

\section{References}

[1] Benkart G., Gregory Th., Premet A., The recognition theorem for graded Lie algebras in prime characteristic, Mem. Amer. Math. Soc. 197 (2009), no. 920, math.RA/0508373.

[2] Bouarroudj S., Grozman P., Lebedev A., Leites D., Divided power (co)homology. Presentations of simple finite dimensional modular Lie superalgebras with Cartan matrix, in preparation.

[3] Bouarroudj S., Grozman P., Leites D., Cartan matrices and presentations of Elduque and Cunha simple Lie superalgebras, see [37], math.RT/0611391.

[4] Bouarroudj S., Grozman P., Leites D., Cartan matrices and presentations of the exceptional simple Elduque Lie superalgebra, see [37], math.RT/0611392.

[5] Bouarroudj S., Grozman P., Leites D., New simple modular Lie superalgebras as generalized prolongations, Funktsional. Anal. i Prilozhen. 42 (2008), no. 3, 1-9 (English transl.: Funct. Anal. Appl. 42 (2008), no. 3, 161-168, math.RT/0704.0130.

[6] Bouarroudj S., Grozman P., Leites D., Deformations of the simple symmetric modular Lie superalgebras, see [37], in preparation.

[7] Bouarroudj S., Grozman P., Leites D., New simple modular Lie algebras in characteristic 2 as generalized prolongs, in preparation.

[8] Bourbaki N., Lie groups and Lie algebras, Chapters 4-6, Translated from the 1968 French original by Andrew Pressley, Elements of Mathematics (Berlin), Springer-Verlag, Berlin, 2002.

[9] Brown G., Properties of a 29-dimensional simple Lie algebra of characteristic three, Math. Ann. 261 (1982), 487-492.

[10] Cartan É., Über die einfachen Transformationsgrouppen, Leipziger Berichte (1893), 395-420, Reprinted in (Euvres complètes, Partie II (French) [Complete works. Part II], Algèbre, systèmes différentiels et problèmes d'équivalence, Éditions du Centre National de la Recherche Scientifique (CNRS), Paris, 1984.

[11] Chapovalov D., Chapovalov M., Lebedev A., Leites D., The classification of almost affine (hyperbolic) Lie superalgebras, J. Nonlinear Math. Phys., to appear, arXiv:0906.1860.

[12] Cohen A.M., Roozemond D.A., Computing Chevalley bases in small characteristics, arXiv:0901.1717.

[13] Cunha I., Elduque A., An extended Freudenthal magic square in characteristic 3, J. Algebra 317 (2007), 471-509, math.RA/0605379.

[14] Cunha I., Elduque A., The extended Freudenthal magic square and Jordan algebras, Manuscripta Math. 123 (2007), 325-351, math.RA/0608191.

[15] Djoković D.Ž., Hochschild G., Semisimplicity of 2-graded Lie algebras. II, Illinois J. Math. 20 (1976), 134143.

Djoković D.Ž., Classification of some 2-graded Lie algebras, J. Pure Appl. Algebra 7 (1976), 217-230. Djoković D.Ž., Isomorphism of some simple 2-graded Lie algebras, Canad. J. Math. 29 (1977), 289-294.

[16] Dzhumadildaev A., 10-commutators, 13-commutators, and odd derivations, J. Nonlinear Math. Phys. 15 (2008), 87-103, math-ph/0603054.

[17] Elduque A., New simple Lie superalgebras in characteristic 3, J. Algebra 296 (2006), 196-233, math.RA/0412395. 
[18] Elduque A., Some new simple modular Lie superalgebras, Pacific J. Math. 231 (2007), 337-359, math.RA/0512654.

[19] Elduque A., Models of some simple modular Lie superalgebras, Pacific J. Math. 240 (2009), 49-83, arXiv:0805.1304.

[20] Feigin B., Leites D., Serganova V., Kac-Moody superalgebras, in Group-Theoretical Methods in Physics (Zvenigorod, 1982), Editors M. Markov et al., Nauka, Moscow, 1983, Vol. 1, 274-278 (English transl.: Harwood Academic Publ., Chur, 1985, Vols. 1-3, 631-637).

[21] Frappat L., Sciarrino A., Sorba P., Dictionary on Lie Superalgebras, with 1 CD-ROM (Windows, Macintosh and UNIX), Academic Press, Inc., San Diego, CA, 2000, hep-th/9607161.

[22] Freund P., Kaplansky I., Simple supersymmetries, J. Math. Phys. 17 (1976), 228-231.

[23] Frohardt D., Griess R.L. Jr., Automorphisms of modular Lie algebras, Nova J. Algebra Geom. 1 (1992), 339-345.

[24] Fulton W., Harris J., Representation theory. A first course, Graduate Texts in Mathematics, Vol. 129, Readings in Mathematics, Springer-Verlag, New York, 1991.

[25] Grozman P., SuperLie, http://www.equaonline.com/math/SuperLie.

[26] Hoyt C., Serganova V., Classification of finite-growth general Kac-Moody superalgebras, Comm. Algebra 35 (2007), 851-874, arXiv:0810.2637.

[27] Jacobson N., Lie algebras, Dover Publications, Inc., New York, 1979.

[28] Kac V.G., Infinite-dimensional Lie algebras, 3rd ed., Cambridge University Press, Cambridge, 1990.

[29] Kac V.G., Lie superalgebras, Adv. Math. 26 (1977), 8-96.

[30] Kac V.G., Corrections to: "Exponentials in Lie algebras of characteristic p" [Izv. Akad. Nauk SSSR 35 (1971), no. 4, 762-788] by B. Yu. Veisfeiler and V.G. Kac, Izv. Ross. Akad. Nauk Ser. Mat. 58 (1994), no. 4, 224 (English transl.: Russian Acad. Sci. Izv. Math. 45 (1995), no. 1, 229).

[31] Kaplansky I., Graded Lie algebras, Preprints, University of Chicago, Chicago, 1975, available at http://justpasha.org/math/links/subj/lie/kaplansky.

[32] Kaplansky I., Superalgebras, Pacific J. Math. 86 (1980), 93-98.

[33] Kochetkov Yu., Leites D., Simple finite dimensional Lie algebras in characteristic 2 related to superalgebras and on a notion of finite simple group, in Proceedings of the International Conference on Algebra (Novosibirsk, August 1989), Editors L.A. Bokut, Yu.L. Ershov and A.I. Kostrikin, Contemp. Math., Vol. 131, Part 2, Amer. Math. Soc., Providence, RI, 1992, 59-67.

[34] Lebedev A., Non-degenerate bilinear forms in characteristic 2, related contact forms, simple Lie algebras and superalgebras, math.AC/0601536.

[35] Leites D., Towards classification of simple finite dimensional modular Lie superalgebras in characteristic $p$, J. Prime Res. Math. 3 (2007), 101-110, arXiv:0710.5638.

[36] Leites D. (Editor), Seminar on Supermanifolds, Reports of the Department of Mathematics of Stockholm University, nn. 1-34, 1986-1990, 2100 pages (a version in Russian is to appear in MCCME, 2009; a version in English in preparation).

[37] Leites D. (Editor), Representation theory, Vol. 2: Nonholonomic distributions in representation theory. Quest for simple modular Lie algebras (S. Bouarroudj, B. Clarke, P. Grozman, A. Lebedev, D. Leites, I. Shchepochkina), A. Salam School of Mathematical Sciences, Lahore, 2009.

[38] Leites D., Saveliev M. V., Serganova V.V., Embeddings of $\mathfrak{o s p}(N \mid 2)$ and completely integrable systems, in Proceedings of International Seminar Group-Theoretical Methods in Physics (Yurmala, May 1985), Editors V. Dodonov and V. Man'ko, Nauka, Moscow, 1986, 377-394 (an enlarged version in English is published by VNU Sci Press, 1986, 255-297).

[39] Leites D., Shchepochkina I., Classification of simple Lie superalgebras of vector fields, preprint MPIM-200328, available at http://www.mpim-bonn.mpg.de.

[40] Leites D., Shchepochkina I., How the antibracket should be quantized?, Teoret. Mat. Fiz. 126 (2001), 339-369 (English transl.: Theoret. and Math. Phys. 126 (2001), 281-306), math-ph/0510048.

[41] Onishchik A.L., Vinberg È.B., Lie groups and algebraic groups, Translated from the Russian and with a preface by D.A. Leites, Springer Series in Soviet Mathematics, Springer-Verlag, Berlin, 1990.

[42] Premet A., Strade H., Simple Lie algebras of small characteristic VI. Completion of the classification, arXiv:0711.2899. 
[43] ftp.mccme.ru/users/protopopov/dyno

[44] Ray U., Automorphic forms and Lie superalgebras, Algebras and Applications, Vol. 5, Springer, Dordrecht, 2006.

[45] Nahm W., Rittenberg V., Scheunert M., The classification of graded Lie algebras, Phys. Lett. B 61 (1976), 383-384.

Scheunert M., Nahm W., Rittenberg V., Classification of all simple graded Lie algebras whose Lie algebra is reductive. I, J. Math. Phys. 17 (1976), 1626-1639.

Scheunert M., Nahm W., Rittenberg V., Classification of all simple graded Lie algebras whose Lie algebra is reductive. II. Construction of the exceptional algebras, J. Math. Phys. 17 (1976), 1640-1644.

[46] Sergeev A., Orthogonal polynomials and Lie superalgebras, math.RT/9810110.

[47] Serganova V., Automorphisms of simple Lie superalgebras, Izv. Akad. Nauk SSSR Ser. Mat. 48 (1984), 585-598 (English transl.: Math. USSR-Izv. 24 (1985), 539-551).

[48] Serganova V., On generalizations of root systems, Comm. Algebra 24 (1996), 4281-4299.

[49] Shchepochkina I., How to realize Lie algebras by vector fields, Teoret. Mat. Fiz. 147 (2006), 450-469 (English transl.: Theoret. and Math. Phys. 147 (2006), 821-838), math.RT/0509472.

[50] Skryabin S.M., A contragredient 29-dimensional Lie algebra of characteristic 3, Sibirsk. Mat. Zh. 34 (1993), no. 3, 171-178 (English transl.: Siberian Math. J. 34 (1993), no. 3, 548-554).

[51] Steinberg R., Lectures on Chevalley groups, Notes prepared by John Faulkner and Robert Wilson, Yale University, New Haven, Conn., 1968.

[52] Strade H., Simple Lie algebras over fields of positive characteristic. I. Structure theory, de Gruyter Expositions in Mathematics, Vol. 38, Walter de Gruyter \& Co., Berlin, 2004.

[53] van de Leur J., A classification of contragredient Lie superalgebras of finite growth, Comm. Algebra 17 (1989), 1815-1841.

[54] Weisfeiler B.Ju., Kac V.G., Exponentials in Lie algebras of characteristic p, Izv. Akad. Nauk SSSR Ser. Mat. 35 (1971), 762-788 (in Russian). 